This is a self-archived version of an original article. This version may differ from the original in pagination and typographic details.

Author(s): Parkkonen, Jouni; Paulin, Frédéric

Title: Counting common perpendicular arcs in negative curvature

Year: 2017

Version: Accepted version (Final draft)

Copyright: @ Cambridge University Press, 2016

Rights: In Copyright

Rights url: http://rightsstatements.org/page//nC/1.0/?language=en

Please cite the original version:

Parkkonen, J., \& Paulin, F. (2017). Counting common perpendicular arcs in negative curvature. Ergodic Theory and Dynamical Systems, 37(3), 900-938. https://doi.org/10.1017/etds.2015.77 


\title{
Counting common perpendicular arcs in negative curvature
}

\author{
Jouni Parkkonen Frédéric Paulin
}

July 27, 2015

\begin{abstract}
Let $D^{-}$and $D^{+}$be properly immersed closed locally convex subsets of a Riemannian manifold with pinched negative sectional curvature. Using mixing properties of the geodesic flow, we give an asymptotic formula as $t \rightarrow+\infty$ for the number of common perpendiculars of length at most $t$ from $D^{-}$to $D^{+}$, counted with multiplicities, and we prove the equidistribution in the outer and inner unit normal bundles of $D^{-}$and $D^{+}$of the tangent vectors at the endpoints of the common perpendiculars. When the manifold is compact with exponential decay of correlations or arithmetic with finite volume, we give an error term for the asymptotic. As an application, we give an asymptotic formula for the number of connected components of the domain of discontinuity of Kleinian groups as their diameter goes to 0 . $\square$
\end{abstract}

\section{Introduction}

Let $M$ be a complete connected Riemannian manifold with pinched sectional curvature at most -1 whose fundamental group is not virtually nilpotent, let $\left(g^{t}\right)_{t \in \mathbb{R}}$ be its geodesic flow. Let $D^{-}$and $D^{+}$be proper nonempty properly immersed closed locally convex subsets of $M$. A common perpendicular from $D^{-}$to $D^{+}$is a locally geodesic path in $M$ starting perpendicularly from $D^{-}$and arriving perpendicularly to $D^{+}$(see Section 2.3 for explanations when the boundary of $D^{-}$or $D^{+}$is not smooth. For all $t>0$, we denote by $\operatorname{Perp}\left(D^{-}, D^{+}, t\right)$ the set of common perpendiculars from $D^{-}$to $D^{+}$with length at most $t$ (considered with multiplicities), and by $\mathscr{N}_{D^{-}, D^{+}}(t)$ its cardinality. We refer to Section 3.3 for the definition of the multiplicities, which are equal to 1 if $D^{-}$and $D^{+}$are embedded and disjoint.

In this paper, we give a general asymptotic formula $\mathscr{N}_{D^{-}, D^{+}}(t) \sim c e^{c^{\prime} t}$ as $t \rightarrow+\infty$, with error term estimates, and we prove the equidistribution of the initial and terminal tangent vectors of the common perpendiculars in the outer and inner unit normal bundles of $D^{-}$and $D^{+}$, respectively. The constants $c, c^{\prime}$ are explicit in terms of the Bowen-Margulis measure $m_{\mathrm{BM}}$ of $M$ and the skinning measures $\sigma_{D^{ \pm}}^{\mp}$ of $D^{ \pm}$. These measures are appropriate pushforwards of the Patterson-Sullivan densities to the unit normal bundles of the lifts of $D^{-}$and $D^{+}$in the universal cover of $M$ described in the present generality for the outer normal bundle in [PP5, generalising OS1, OS2] where $M$ has constant curvature and $D^{-}, D^{+}$are balls, horoballs or totally geodesic submanifolds.

\footnotetext{
${ }^{1}$ Keywords: counting, geodesic arc, convexity, common perpendicular, equidistribution, mixing, decay of correlation, negative curvature, skinning measure, Bowen-Margulis measure, Kleinian groups. AMS codes: $37 \mathrm{D} 40,37 \mathrm{~A} 25,53 \mathrm{C} 22,30 \mathrm{~F} 40$
} 
We now state our counting and equidistribution results. We avoid any compactness assumption on $M$, we only assume that the Bowen-Margulis measure of $M$ is finite and that it is mixing for the geodesic flow. We denote the total mass of any measure $m$ by $\|m\|$. Let $\delta$ be the critical exponent of the fundamental group of $M$.

Theorem 1 Assume that the skinning measures $\sigma_{D^{-}}^{+}$and $\sigma_{D^{+}}^{-}$are finite and nonzero. Then, as $s \rightarrow+\infty$,

$$
\mathscr{N}_{D^{-}, D^{+}}(s) \sim \frac{\left\|\sigma_{D^{-}}^{+}\right\|\left\|\sigma_{D^{+}}^{-}\right\|}{\left\|m_{\mathrm{BM}}\right\|} \frac{e^{\delta s}}{\delta} .
$$

We refer to [DOP] for a finiteness criteria of $m_{\mathrm{BM}}$, to [PP5] for finiteness criterion of the skinning measures, generalising [OS2], and to [Bab] for mixing criteria of $m_{\mathrm{BM}}$.

The counting function $\mathscr{N}_{D^{-}, D^{+}}$has been studied for particular triples $\left(M, D^{-}, D^{+}\right)$ at least since the 1950's for example in [Hub2], [Her, [Mar, [EM], Cos, [Rob], [HP2], [Kon, [KO], [OS1], [PP3], Kim, [Pol] and [OS3]. See [PP8] for a more detailed review.

When $M$ is a finite volume hyperbolic manifold, we get very explicit forms of the counting results also in cases that were not known before, see Corollary 21. For example, if $D^{-}$and $D^{+}$are closed geodesics of $M$ of lengths $\ell_{-}$and $\ell_{+}$, respectively, then the number of common perpendiculars from $D^{-}$to $D^{+}$of length at most $s$ satisfies, as $s \rightarrow+\infty$,

$$
\mathscr{N}_{D^{-}, D^{+}}(s) \sim \frac{\pi^{\frac{n}{2}-1} \Gamma\left(\frac{n-1}{2}\right)^{2}}{2^{n-2}(n-1) \Gamma\left(\frac{n}{2}\right)} \frac{\ell_{-} \ell_{+}}{\operatorname{Vol}(M)} e^{(n-1) s} .
$$

When $M$ is a closed surface and $D^{-}=D^{+}$, the formula (1) is proved in [MaMW] by trace formula methods, but obtaining the case $D^{-} \neq D^{+}$seems difficult by their methods.

We denote the initial and terminal unit tangent vectors of $\alpha \in \operatorname{Perp}\left(D^{-}, D^{+}, t\right)$ by $v_{\alpha}^{-}$ and $v_{\alpha}^{+}$, and the unit Dirac mass at a point $z$ by $\Delta_{z}$. Theorem 1 is deduced in Section 3 from the following joint equidistribution result of these vectors towards the skinning measures of $D^{-}$and $D^{+}$, that generalises work of Herrmann and Roblin for special $D^{ \pm}$'s.

Theorem 2 For the weak-star convergence of measures on $T^{1} M \times T^{1} M$, we have

$$
\lim _{t \rightarrow+\infty} \delta\left\|m_{\mathrm{BM}}\right\| e^{-\delta t} \sum_{\alpha \in \operatorname{Perp}\left(D^{-}, D^{+}, t\right)} \Delta_{v_{\alpha}^{-}} \otimes \Delta_{v_{\alpha}^{+}}=\sigma_{D^{-}}^{+} \otimes \sigma_{D^{+}}^{-} .
$$

Both results are valid when $M$ is a good orbifold instead of a manifold (for the appropriate notion of multiplicities), and when $D^{-}, D^{+}$are replaced by locally finite families.

Besides giving a unified treatment that covers all the special cases in the above references, we have very weak finiteness and curvature assumptions on the manifold, and no regularity assumptions on the convex sets (see Corollary 4 for a striking application using convex sets with fractal boundary). We develop new techniques (necessary for the generality considered in this paper) in order to apply Margulis's idea to use the mixing of the geodesic flow: Due to the symmetry of the problem, a one-sided pushing of the geodesic flow is not sufficient and we push simultaneously the outer/inner unit normal vectors to the convex sets in opposite directions. We also need a new effective study of the geometry and the dynamics of the creation of common perpendiculars, see Subsection 2.3 .

In the cases when the geodesic flow is known to be exponentially mixing on $T^{1} M$, we obtain an exponentially small error term in the approximation of the counting function $\mathscr{N}_{D^{-}, D^{+}}$. In particular, when $M$ is arithmetic, the error term in Equation (1) is $\mathrm{O}\left(e^{(n-1-\kappa) t}\right)$ for some $\kappa>0$. 
Theorem 3 Assume that $M$ is compact and the geodesic flow is exponentially mixing for the Hölder regularity, or that $M$ is locally symmetric, the boundary of $D^{ \pm}$is smooth, $m_{\mathrm{BM}}$ is finite, smooth, and exponentially mixing under the geodesic flow for the Sobolev regularity. Assume that the strong stable/unstable ball masses by the conditionals of $m_{\mathrm{BM}}$ are Hölder-continuous in their radius. Then there exists $\kappa>0$ such that, as $t \rightarrow+\infty$,

$$
\mathscr{N}_{D^{-}, D^{+}, F}(t)=\frac{\left\|\sigma_{D^{-}}^{+}\right\|\left\|\sigma_{D^{+}}^{-}\right\|}{\delta\left\|m_{\mathrm{BM}}\right\|} e^{\delta t}\left(1+\mathrm{O}\left(e^{-\kappa t}\right)\right) .
$$

See Section 4 for a discussion of the assumptions and the dependence of $\mathrm{O}(\cdot)$ on the data. Similar (sometimes more precise) error estimates were known earlier for the counting function in special cases of $D^{ \pm}$in constant curvature geometrically finite manifolds (often in small dimension) through the work of Huber, Selberg, Patterson, Lax-Phillips [LaP], Cosentino [Cos, Kontorovich-Oh [KO], Lee-Oh [LO].

Consider the picture above produced by D. Wright's program kleinian, which is the limit set of a free product $\Gamma=\Gamma_{0} * \gamma_{0} \Gamma_{0} \gamma_{0}^{-1}$ of a quasifuchsian group $\Gamma_{0}$ and its conjugate by a large power $\gamma_{0}$ of a loxodromic element whose attracting fixed point is contained in the bounded component of $\mathbb{C}-\Lambda \Gamma$, so that the limit set of $\Gamma$ is the closure of a countable union of quasi-circles. As we will see in Section 5, the number of Jordan curves in $\Lambda \Gamma$ with diameter at least $1 / T$ is equivalent to $c T^{\delta}$ where $c>0$ and $\left.\delta \in\right] 1,2[$ is the Hausdorff dimension of the limit set.

Corollary 4 Let $\Gamma$ be a geometrically finite discrete group of isometries of the upper halfspace model of $\mathbb{H}_{\mathbb{R}}^{n}$, with bounded limit set $\Lambda \Gamma$ in $\mathbb{R}^{n-1}=\partial_{\infty} \mathbb{H}_{\mathbb{R}}^{n}-\{\infty\}$. Let $\delta$ be the Hausdorff dimension of $\Lambda \Gamma$. Let $\Gamma_{0}$ be a convex-cocompact subgroup of $\Gamma$ with infinite index. Then, there exists an explicitable $c>0$ such that, as $T \rightarrow+\infty$,

$$
\operatorname{Card}\left\{\gamma \in \Gamma / \Gamma_{0}: \operatorname{diam}\left(\gamma \Lambda \Gamma_{0}\right) \geq 1 / T\right\} \sim c T^{\delta} .
$$

This corollary is due to OS3 when the limit set of $\Gamma_{0}$ is a round sphere (allowing the use of homogeneous dynamics). We refer to Corollary 17 for a more general version and to Section 5 for complements and for extensions to any rank one symmetric space. 
The results of this paper have been announced in the survey [PP8]. In [PP6], we give several arithmetic applications of the results of this paper, obtained by considering arithmetically defined manifolds and orbifolds of constant negative curvature. In [PP9], we consider arithmetic applications in the Heisenberg group via complex hyperbolic geometry. In [PP7], we study counting and equidistribution in conjugacy classes, giving a new proof of the main result of [Hub1] and generalising it to parabolic cyclic and more general subgroups, arbitrary dimension, infinite volume and variable curvature.

The previous ArXiv version of this article contained the extension of the counting and equidistribution results 1, 2 and 3 to Gibbs measures (that is, equilibrium states associated with Hölder potentials on $\left.T^{1} M\right)$ and counting functions with weights. In order to shorten this paper, this material will appear as part of [BPP].

Acknowledgement: The first author thanks the Universite Paris-Sud for a month of visiting professor where this work was started, and the FIM of ETH Zürich for its support when this work was continued. The second author thanks ETH Zürich for frequent stays during the writing of this paper. Both authors thank the Mittag-Leffler Institute where this paper was almost completed.

\section{Geometry, dynamics and convexity}

Let $\widetilde{M}$ be a complete simply connected Riemannian manifold with (dimension at least 2 and) pinched negative sectional curvature $-b^{2} \leq K \leq-1$, and let $x_{0} \in \widetilde{M}$ be a fixed basepoint. Let $\Gamma$ be a nonelementary (not virtually nilpotent) discrete group of isometries

of $\widetilde{M}$, and let $M$ be the quotient Riemannian orbifold $\Gamma \backslash \widetilde{M}$. We denote by $\partial_{\infty} \widetilde{M}$ the boundary at infinity of $\widetilde{M}$, by $\delta=\lim _{t \rightarrow+\infty} \frac{1}{t} \operatorname{Card}\left\{\gamma \in \Gamma: d\left(x_{0}, \gamma x_{0}\right) \leq t\right\}$ the critical exponent of $\Gamma$, and by $\Lambda \Gamma$ the limit set of $\Gamma$.

In this section, we review the required background on negatively curved Riemannian manifolds seen as locally CAT $(-\kappa)$ spaces (see $[\mathrm{BrH}]$ for definitions, proofs and complements). We introduce the notation for the outward and inward pointing unit normal bundles of the boundary of a convex subset, and we define dynamical thickenings in the unit tangent bundle of subsets of these submanifolds, expanding on [PP5]. We give a precise definition of common perpendiculars in Subsection 2.3 and we also give a procedure to construct them by dynamical means.

For every $\epsilon>0$, we denote by $\mathscr{N}_{\epsilon} A$ the closed $\epsilon$-neighbourhood of a subset $A$ of any metric space, by $\mathscr{N}_{-\epsilon} A$ the set of points $x \in A$ at distance at least $\epsilon$ from the complement of $A$, and by convention $\mathscr{N}_{0} A=\bar{A}$.

\subsection{Strong stable and unstable foliations, and Hamenstädt's distances}

We identify the unit tangent bundle $T^{1} N$ (endowed with Sasaki's Riemannian metric) of a complete Riemannian manifold $N$ with the set of its locally geodesic lines $\ell: \mathbb{R} \rightarrow N$, by the inverse of the map sending a (locally) geodesic line $\ell$ to its (unit) tangent vector $\dot{\ell}(0)$ at time $t=0$. We denote by $\pi: T^{1} N \rightarrow N$ the basepoint projection, given by $\pi(\ell)=\ell(0)$.

The geodesic flow on $T^{1} N$ is the smooth one-parameter group of diffeomorphisms $\left(g^{t}\right)_{t \in \mathbb{R}}$ of $T^{1} \widetilde{M}$, where $g^{t} \ell(s)=\ell(s+t)$, for all $\ell \in T^{1} N$ and $s, t \in \mathbb{R}$. The action of the isometry group of $N$ on $T^{1} N$ by postcomposition (that is, by $(\gamma, \ell) \mapsto \gamma \circ \ell$ ) commutes with the geodesic flow. 
When $\Gamma$ acts without fixed points on $\widetilde{M}$, we have an identification $\Gamma \backslash T^{1} \widetilde{M}=T^{1} M$. More generally, we denote by $T^{1} M$ the quotient Riemannian orbifold $\Gamma \backslash T^{1} \widetilde{M}$. We use the notation $\left(g^{t}\right)_{t \in \mathbb{R}}$ also for the (quotient) geodesic flow on $T^{1} M$.

For every $v \in T^{1} \widetilde{M}$, let $v_{-} \in \partial_{\infty} \widetilde{M}$ and $v_{+} \in \partial_{\infty} \widetilde{M}$, respectively, be the endpoints at $-\infty$ and $+\infty$ of the geodesic line defined by $v$. Let $\partial_{\infty}^{2} \widetilde{M}$ be the subset of $\partial_{\infty} \widetilde{M} \times \partial_{\infty} \widetilde{M}$ which consists of pairs of distinct points at infinity of $\widetilde{M}$. Hopf's parametrisation of $T^{1} \widetilde{M}$ is the homeomorphism which identifies $T^{1} \widetilde{M}$ with $\partial_{\infty}^{2} \widetilde{M} \times \mathbb{R}$, by the map $v \mapsto\left(v_{-}, v_{+}, t\right)$, where $t$ is the signed distance of the closest point to $x_{0}$ on the geodesic line defined by $v$ to $\pi(v)$. We have $g^{s}\left(v_{-}, v_{+}, t\right)=\left(v_{-}, v_{+}, t+s\right)$ for all $s \in \mathbb{R}$, and for all $\gamma \in \Gamma$, we have $\gamma\left(v_{-}, v_{+}, t\right)=\left(\gamma v_{-}, \gamma v_{+}, t+t_{\gamma, v_{-}, v_{+}}\right)$where $t_{\gamma, v_{-}, v_{+}} \in \mathbb{R}$ depends only on $\gamma, v_{-}, v_{+}$.

Let $\iota: T^{1} \widetilde{M} \rightarrow T^{1} \widetilde{M}$ be the antipodal (flip) map of $T^{1} \widetilde{M}$ defined by $\iota v=-v$ or, using geodesic lines, by $\iota v: t \mapsto v(-t)$. In Hopf's parametrisation, the antipodal map is the map $\left(v_{-}, v_{+}, t\right) \mapsto\left(v_{+}, v_{-},-t\right)$. We denote the quotient map of $\iota$ again by $\iota: T^{1} M \rightarrow T^{1} M$, and call it the antipodal map of $T^{1} M$. We have $\iota \circ g^{t}=g^{-t} \circ \iota$ for all $t \in \mathbb{R}$.

The strong stable/unstable manifold of $v \in T^{1} \widetilde{M}$ is

$$
W^{ \pm}(v)=\left\{v^{\prime} \in T^{1} \widetilde{M}: d\left(v(t), v^{\prime}(t)\right) \rightarrow 0 \text { as } t \rightarrow \pm \infty\right\} .
$$

The union for $t \in \mathbb{R}$ of the images under $g^{t}$ of the strong stable manifold of $v \in T^{1} \widetilde{M}$ is the stable manifold $W^{0+}(v)=\bigcup_{t \in \mathbb{R}} g^{t} W^{+}(v)$ of $v$, which consists of the elements $v^{\prime} \in T^{1} \widetilde{M}$ with $v_{+}^{\prime}=v_{+}$. Similarly, $W^{0-}(v)=\bigcup_{t \in \mathbb{R}} g^{t} W^{-}(v)$, which consists of the elements $v^{\prime} \in$ $T^{1} \widetilde{M}$ with $v_{-}^{\prime}=v_{-}$, is the unstable manifold $W^{0-}(v)$ of $v$. The maps from $\mathbb{R} \times W^{ \pm}(v)$ to $W^{0 \pm}(v)$ defined by $\left(s, v^{\prime}\right) \mapsto g^{s} v^{\prime}$ are smooth diffeomorphisms.

The strong stable manifolds, stable manifolds, strong unstable manifolds and unstable manifolds are the (smooth) leaves of topological foliations that are invariant under the geodesic flow and the group of isometries of $\widetilde{M}$, denoted by $\mathscr{W}^{+}, \mathscr{W}^{0+}, \mathscr{W}^{-}$and $\mathscr{W}^{0-}$ respectively. These foliations are Hölder-continuous when $\widetilde{M}$ has compact quotients or when $\widetilde{M}$ has pinched negative sectional curvature with bounded derivatives (see for instance [Bri], [PPS, Thm. 7.3]) and even smooth when $\widetilde{M}$ is symmetric.

For any point $\xi \in \partial_{\infty} \widetilde{M}$, let $\rho_{\xi}:\left[0,+\infty\left[\rightarrow \widetilde{M}\right.\right.$ be the geodesic ray with origin $x_{0}$ and point at infinity $\xi$. The Busemann cocycle of $\widetilde{M}$ is the map $\beta: \widetilde{M} \times \widetilde{M} \times \partial_{\infty} \widetilde{M} \rightarrow \mathbb{R}$ defined by $(x, y, \xi) \mapsto \beta_{\xi}(x, y)=\lim _{t \rightarrow+\infty} d\left(\rho_{\xi}(t), x\right)-d\left(\rho_{\xi}(t), y\right)$.

The projections in $\widetilde{M}$ of the strong unstable and strong stable manifolds of $v \in T^{1} \widetilde{M}$, denoted by $H_{-}(v)=$ $\pi\left(W^{-}(v)\right)$ and $H_{+}(v)=\pi\left(W^{+}(v)\right)$, are the unstable and stable horospheres of $v$ centered at $v_{-}$and $v_{+}$, respectively. The unstable horosphere of $v$ coincides with the zero set of the map $x \mapsto f_{-}(x)=\beta_{v_{-}}(x, \pi(v))$ and the stable horosphere of $v$ is the zero set of $x \mapsto f_{+}(x)=\beta_{v_{+}}(x, \pi(v))$. The sublevel sets $\left.\left.H B_{-}(v)=f_{-}^{-1}(]-\infty, 0\right]\right)$ and $H B_{+}(v)=$ $\left.\left.f_{+}^{-1}(]-\infty, 0\right]\right)$ are the horoballs bounded by $H_{-}(v)$ and $v_{-} \quad v \quad v_{+}$ $H_{-}(v) \quad H_{+}(v)$ $H_{+}(v)$. Horoballs are (strictly) convex subsets of $\widetilde{M}$.

For every $v \in T^{1} \widetilde{M}$, let $d_{W^{-}(v)}$ and $d_{W^{+}(v)}$ be Hamenstädt's distances on the strong unstable and strong stable leaf of $v$, defined as follows (see for instance [HP1, Appendix]): for all $w, z \in W^{\mp}(v)$, let

$$
d_{W^{\mp}(v)}(w, z)=\lim _{t \rightarrow+\infty} e^{\frac{1}{2} d(w( \pm t), z( \pm t))-t} .
$$


Hamenstädt's distances are distances inducing the original topology on $W^{ \pm}(v)$. For all $w, z \in W^{ \pm}(v)$ and $s \in \mathbb{R}$, and for every isometry $\gamma$ of $\widetilde{M}$, we have

$$
d_{W^{ \pm}(\gamma v)}(\gamma w, \gamma z)=d_{W^{ \pm}(v)}(w, z) \quad \text { and } \quad d_{W^{ \pm}\left(g^{s} v\right)}\left(g^{s} w, g^{s} z\right)=e^{\mp s} d_{W^{ \pm}(v)}(w, z) .
$$

A proof of the following result, first given in a preliminary version of this paper, may now be found in [PPS, Lem. 2.4].

Lemma 5 For all $v \in T^{1} \widetilde{M}, v^{\prime} \in W^{ \pm}(v)$, we have

$$
d\left(\pi(v), \pi\left(v^{\prime}\right)\right) \leq d_{W^{ \pm}(v)}\left(v, v^{\prime}\right)
$$

\subsection{Dynamical thickening of outer and inner unit normal bundles}

Let $D$ be a nonempty proper closed convex subset in $\widetilde{M}$. We denote by $\partial D$ its boundary in $\widetilde{M}$ and by $\partial_{\infty} D$ its set of points at infinity. In this subsection, we recall from [PP5] the definition of the outer unit normal bundle of $\partial D$, the dynamical thickenings of its subsets, and we extend these definitions to the inner unit normal bundle of $\partial D$.

Let $P_{D}: \widetilde{M} \cup\left(\partial_{\infty} \widetilde{M}-\partial_{\infty} D\right) \rightarrow D$ be the (continuous) closest point map defined on $\xi \in \partial_{\infty} \widetilde{M}-\partial_{\infty} D$ by setting $P_{D}(\xi)$ to be the unique point in $D$ that minimises the function $y \mapsto \beta_{\xi}\left(y, x_{0}\right)$ from $D$ to $\mathbb{R}$. The outer unit normal bundle $\partial_{+}^{1} D$ of the boundary of $D$ is the topological submanifold of $T^{1} \widetilde{M}$ consisting of the geodesic lines $v: \mathbb{R} \rightarrow \widetilde{M}$ with $P_{D}\left(v_{+}\right)=v(0)$. The inner unit normal bundle of the boundary of $D$ is $\partial_{-}^{1} D=\iota \partial_{+}^{1} D$. Note that $\pi\left(\partial_{ \pm}^{1} D\right)=\partial D$, that $\partial_{+}^{1} H B_{-}(v)$ is the strong unstable manifold $W^{-}(v)$ of $v$ and that $W^{+}(v)=\partial_{-}^{1} H B_{+}(v)$. When $D$ is a totally geodesic submanifold of $\widetilde{M}$, then $\partial_{+}^{1} D=\partial_{-}^{1} D$.

The restriction of $P_{D}$ to $\partial_{\infty} \widetilde{M}-\partial_{\infty} D$ is not injective in general, but the inverse $P_{D}^{+}$ of the restriction to $\partial_{+}^{1} D$ of the (positive) endpoint map $v \mapsto v_{+}$is a natural lift of $P_{D}$ to a homeomorphism from $\partial_{\infty} \widetilde{M}-\partial_{\infty} D$ to $\partial_{+}^{1} D$ such that $\pi \circ P_{D}^{+}=P_{D}$. Similarly, $P_{D}^{-}=\iota \circ P_{D}^{+}: \partial_{\infty} \widetilde{M}-\partial_{\infty} D \rightarrow \partial_{-}^{1} D$ is a homeomorphism such that $\pi \circ P_{D}^{-}=P_{D}$.

For every isometry $\gamma$ of $\widetilde{M}$, we have $\partial_{ \pm}^{1}(\gamma D)=\gamma \partial_{ \pm}^{1} D$ and $P_{\gamma D}^{ \pm} \circ \gamma=\gamma \circ P_{D}^{ \pm}$. In particular, $\partial_{ \pm}^{1} D$ is invariant under the isometries of $\widetilde{M}$ that preserve $D$. For all $t \geq 0$, we have $g^{ \pm t} \partial_{ \pm}^{1} D=\partial_{ \pm}^{1}\left(\mathscr{N}_{t} D\right)$.

We define

$$
\mathscr{U}_{D}^{ \pm}=\left\{v \in T^{1} \widetilde{M}: v_{ \pm} \notin \partial_{\infty} D\right\}
$$

Note that $\mathscr{U}_{D}^{-}=\iota \mathscr{U}_{D}^{+}$, and that $\mathscr{U}_{D}^{ \pm}$is an open subset of $T^{1} \widetilde{M}$, invariant under the geodesic flow. We have $\mathscr{U}_{\gamma D}^{ \pm}=\gamma \mathscr{U}_{D}^{ \pm}$for every isometry $\gamma$ of $\widetilde{M}$ and, in particular, $\mathscr{U}_{D}^{ \pm}$is invariant under the isometries of $\widetilde{M}$ preserving $D$.

Define a fibration $f_{D}^{+}: \mathscr{U}_{D}^{+} \rightarrow \partial_{+}^{1} D$ as the composition of the positive endpoint map from $\mathscr{U}_{D}^{+}$onto $\partial_{\infty} \widetilde{M}-\partial_{\infty} D$ (which is a fibration) and the homeomorphism $P_{D}^{+}$from $\partial_{\infty} \widetilde{M}-\partial_{\infty} D$ to $\partial_{+}^{1} D$. The fiber of $w \in \partial_{+}^{1} D$ for $f_{D}^{+}$is exactly the stable leaf

$$
W^{0+}(w)=\left\{v \in T^{1} \widetilde{M}: v_{+}=w_{+}\right\}
$$

$D$

$$
\begin{aligned}
f_{D}^{+}(v) & v_{+} \\
= & P_{D}^{+}\left(v_{+}\right)
\end{aligned}
$$


Analogously, we define a fibration $f_{D}^{-}=\iota \circ f_{D}^{+} \circ \iota: \mathscr{U}_{D}^{-} \rightarrow \partial_{-}^{1} D$ as the composition of the negative endpoint map with $P_{D}^{-}$, for which the fiber of $w \in \partial_{-}^{1} D$ is the unstable leaf $W^{0-}(w)=\left\{v \in T^{1} \widetilde{M}: v_{-}=w_{-}\right\}$.

For every isometry $\gamma$ of $\widetilde{M}$, we have

$$
f_{\gamma D}^{ \pm} \circ \gamma=\gamma \circ f_{D}^{ \pm}
$$

We have $f_{\mathscr{N}_{t} D}^{ \pm}=g^{ \pm t} \circ f_{D}^{ \pm}$for all $t \geq 0$, and $f_{D}^{ \pm} \circ g^{t}=f_{D}^{ \pm}$for all $t \in \mathbb{R}$. In particular, the fibrations $f_{D}^{ \pm}$are invariant under the geodesic flow.

The next result will only be used for the error term estimates in Section 4 . Note that if $\widetilde{M}$ is a symmetric space (in which case the strong stable and unstable foliations are smooth, and the sphere at infinity has a smooth structure such that the maps $v \mapsto v_{ \pm}$ from $W^{\mp}(w)$ to $\partial_{\infty} \widetilde{M}-\left\{w_{\mp}\right\}$ are smooth), and if $D$ has smooth boundary, then the fibrations $f_{D}^{ \pm}$are smooth.

Recall that a map $f: X \rightarrow Y$ between two metric spaces is (uniformly locally) Höldercontinuous if there exist $c, c^{\prime}>0$ and $\left.\left.\alpha \in\right] 0,1\right]$ such that $d(f(x), f(y)) \leq c d(x, y)^{\alpha}$ for all $x, y \in X$ with $d(x, y) \leq c^{\prime}$.

Lemma 6 The maps $f_{D}^{ \pm}$are Hölder-continuous on the set of elements $v \in \mathscr{U}_{D}^{ \pm}$such that $d\left(\pi(v), \pi\left(f_{D}^{ \pm}(v)\right)\right)$ is bounded.

Proof. We prove the result for $f_{D}^{+}$, the one for $f_{D}^{-}$follows similarly. For all $u, u^{\prime} \in T^{1} \widetilde{M}$, denote the geodesic lines they define by $t \mapsto u_{t}, u_{t}^{\prime}$, and let

$$
\delta_{1}\left(u, u^{\prime}\right)=\exp \left(-\sup \left\{t \geq 0: \sup _{s \in[-t, t]} d\left(u_{s}, u_{s}^{\prime}\right) \leq 1\right\}\right) \quad \text { and } \quad \delta_{2}\left(u, u^{\prime}\right)=\sup _{t \in[0,1]} d\left(u_{t}, u_{t}^{\prime}\right) .
$$

with the convention $\delta_{1}\left(u, u^{\prime}\right)=1$ if $d\left(u_{0}, u_{0}^{\prime}\right)>1$ and $\delta_{1}\left(u, u^{\prime}\right)=0$ if $u=u^{\prime}$. By for instance [Bal, p. 70], the maps $\delta_{1}, \delta_{2}$ are distances on $T^{1} \widetilde{M}$ which are Hölder-equivalent to Sasaki's distance.

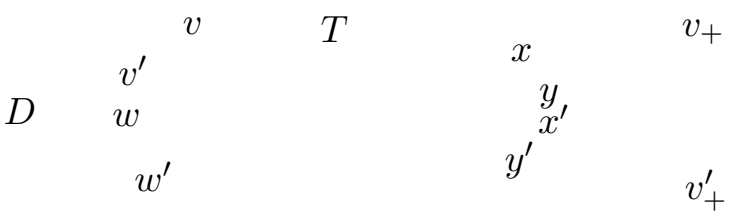

Let $v, v^{\prime} \in T^{1} \widetilde{M}$ be such that $d\left(v_{0}, v_{0}^{\prime}\right) \leq 1$, let $w=f_{D}^{+}(v)$ and $w^{\prime}=f_{D}^{+}\left(v^{\prime}\right)$. Let $T=\sup \left\{t \geq 0: \sup _{s \in[0, t]} d\left(v_{s}, v_{s}^{\prime}\right) \leq 1\right\}$, so that $\delta_{1}\left(v, v^{\prime}\right) \geq e^{-T}$. We may assume that $T$ is finite, otherwise $v_{+}=v_{+}^{\prime}$, hence $w=w^{\prime}$. Let $x=v_{T}$ and $x^{\prime}=v_{T}^{\prime}$, which satisfy $d\left(x, x^{\prime}\right) \leq 1$. Let $y$ (respectively $y^{\prime}$ ) be the closest point to $x$ (respectively $x^{\prime}$ ) on the geodesic ray defined by $w$ (respectively $\left.w^{\prime}\right)$. By convexity, since $d\left(v_{0}, w_{0}\right)$ and $d\left(v_{0}^{\prime}, w_{0}^{\prime}\right)$ are bounded by a constant $c>0$ and since $v_{+}=w_{+}, v_{+}^{\prime}=w_{+}^{\prime}$, we have $d(x, y) \leq c$ and $d\left(x^{\prime}, y^{\prime}\right) \leq c$. By the triangle inequality, we have $d\left(y, y^{\prime}\right) \leq 2 c+1, d\left(y, w_{1}\right) \geq T-2 c-1$ and $d\left(y^{\prime}, w_{1}^{\prime}\right) \geq T-2 c-1$. By convexity, and since projection maps exponentially decrease the distances, there exists a constant $c^{\prime}>0$ such that

$$
\delta_{2}\left(w, w^{\prime}\right)=d\left(w_{1}, w_{1}^{\prime}\right) \leq c^{\prime} d\left(y, y^{\prime}\right) e^{-(T-2 c-1)} \leq c^{\prime}(2 c+1) e^{2 c+1} \delta_{1}\left(v, v^{\prime}\right) .
$$


The result follows.

Let $\eta, \eta^{\prime}>0$. For all $w \in T^{1} \widetilde{M}$, let

$$
B^{ \pm}\left(w, \eta^{\prime}\right)=\left\{v^{\prime} \in W^{ \pm}(w): d_{W^{ \pm}(w)}\left(v^{\prime}, w\right)<\eta^{\prime}\right\}
$$

$D \quad \pi\left(B^{+}\left(w, \eta^{\prime}\right)\right)$

be the open balls of radius $\eta^{\prime}$ centered at $w$ for Hamenstädt's distance in the strong stable/unstable leaves of $w$. Let

$$
V_{w, \eta, \eta^{\prime}}^{ \pm}=\bigcup_{s \in]-\eta, \eta[} g^{s} B^{ \pm}\left(w, \eta^{\prime}\right)
$$

$$
\begin{gathered}
w \\
\pi\left(V_{w, \eta, \eta^{\prime}}^{+}\right)
\end{gathered}
$$

We have $B^{-}\left(w, \eta^{\prime}\right)=\iota B^{+}\left(\iota w, \eta^{\prime}\right)$ hence $V_{w, \eta, \eta^{\prime}}^{-}=\iota V_{\iota w, \eta, \eta^{\prime}}^{+}$. We have

$$
g^{s} B^{ \pm}\left(w, \eta^{\prime}\right)=B^{ \pm}\left(g^{s} w, e^{\mp s} \eta^{\prime}\right) \text { hence } g^{s} V_{w, \eta, \eta^{\prime}}^{ \pm}=V_{g^{s} w, \eta, e^{\mp s} \eta^{\prime}}^{ \pm}
$$

for all $s \in \mathbb{R}$. For every isometry $\gamma$ of $\widetilde{M}$, we have $\gamma B^{ \pm}\left(w, \eta^{\prime}\right)=B^{ \pm}\left(\gamma w, \eta^{\prime}\right)$ and $\gamma V_{w, \eta, \eta^{\prime}}^{ \pm}=$ $V_{\gamma w, \eta, \eta^{\prime}}^{ \pm}$. The map from $]-\eta, \eta\left[\times B^{ \pm}\left(w, \eta^{\prime}\right)\right.$ to $V_{w, \eta, \eta^{\prime}}^{ \pm}$defined by $\left(s, v^{\prime}\right) \mapsto g^{s} v^{\prime}$ is a homeomorphism. For all subsets $\Omega^{-}$of $\partial_{+}^{1} D$ and $\Omega^{+}$of $\partial_{-}^{1} D$, let

$$
\mathscr{V}_{\eta, \eta^{\prime}}^{+}\left(\Omega^{-}\right)=\bigcup_{w \in \Omega^{-}} V_{w, \eta, \eta^{\prime}}^{+} \text {and } \mathscr{V}_{\eta, \eta^{\prime}}^{-}\left(\Omega^{+}\right)=\bigcup_{w \in \Omega^{+}} V_{w, \eta, \eta^{\prime}}^{-} .
$$

For every isometry $\gamma$ of $\widetilde{M}$, we have $\gamma_{\eta}^{ \pm} \eta_{\eta^{\prime}}^{ \pm}\left(\Omega^{\mp}\right)=\mathscr{V}_{\eta, \eta^{\prime}}^{ \pm}\left(\gamma \Omega^{\mp}\right)$ and for every $t \geq 0$, we have

$$
g^{ \pm t} \mathscr{V}_{\eta, \eta^{\prime}}^{ \pm}\left(\Omega^{\mp}\right)=\mathscr{V}_{\eta, e^{-t} \eta^{\prime}}^{ \pm}\left(g^{ \pm t} \Omega^{\mp}\right)
$$

The thickenings (or dynamical neighbourhoods) $\mathscr{V}_{\eta, \eta^{\prime}}^{ \pm}\left(\Omega^{\mp}\right)$ are nondecreasing in $\eta$ and in $\eta^{\prime}$ and their intersections and unions satisfy

$$
\bigcap_{\eta, \eta^{\prime}>0} \mathscr{V}_{\eta, \eta^{\prime}}^{ \pm}\left(\Omega^{\mp}\right)=\Omega^{\mp} \text { and } \bigcup_{\eta, \eta^{\prime}>0} \mathscr{V}_{\eta, \eta^{\prime}}^{ \pm}\left(\partial_{ \pm}^{1} D\right)=\mathscr{U}_{D}^{ \pm}
$$

The restriction of $f_{D}^{ \pm}$to $\mathscr{V}_{\eta, \eta^{\prime}}^{ \pm}\left(\Omega^{\mp}\right)$ is a fibration over $\Omega^{\mp}$, whose fiber over $w \in \Omega^{\mp}$ is the open subset $V_{w, \eta, \eta^{\prime}}^{ \pm}$of the stable/unstable leaf of $w$.

\subsection{Creating common perpendiculars}

For any two closed convex subsets $D^{-}$and $D^{+}$of $\widetilde{M}$, we say that a geodesic arc $\alpha$ : $[0, T] \rightarrow \widetilde{M}$, where $T>0$, is a common perpendicular from $D^{-}$to $D^{+}$if its initial tangent vector $\dot{\alpha}(0)$ belongs to $\partial_{+}^{1} D^{-}$and if its terminal tangent vector $\dot{\alpha}(T)$ belongs to $\partial_{-}^{1} D^{+}$. It is important to think of common perpendiculars as oriented $\operatorname{arcs}\left(\right.$ from $D^{-}$to $D^{+}$). Note that there exists a common perpendicular from $D^{-}$to $D^{+}$if and only if $D^{-}$and $D^{+}$are nonempty and the closures $\overline{D^{-}}$and $\overline{D^{+}}$of $D^{-}$and $D^{+}$in the compactification $\widetilde{M} \cup \partial_{\infty} \widetilde{M}$ are disjoint. A common perpendicular from $D^{-}$to $D^{+}$, if it exists, is unique.

When $\overline{D^{-}}$and $\overline{D^{+}}$are disjoint, and when $\partial D^{-}$and $\partial D^{+}$are $\mathscr{C}^{1}$-submanifolds (for instance, if $D^{ \pm}$are closed $\epsilon$-neighbourhoods of nonempty convex subsets of $\widetilde{M}$ for some $\epsilon>0$, see [Wal]), this definition of a common perpendicular corresponds to the usual one. But there are interesting closed convex subsets with less regular boundary, such as 
in general the convex hulls of limit sets of nonelementary discrete groups of isometries of $\widetilde{M}$. Although it would be possible to take the closed $\epsilon$-neighbourhood, to count common perpendiculars in the usual sense, and then to take a limit as $\epsilon$ goes to 0 , it is more natural to work directly in the above generality (see [PP8, Sect. 3.2] for further comments).

The crucial observation is that two nonempty proper closed convex subsets $D^{-}$and $D^{+}$of $\widetilde{M}$ have a common perpendicular $\alpha$ of length a given $t>0$ if and only if the pushforwards and pullbacks by the geodesic flow at time $\frac{t}{2}$ of the outer and inner normal bundles of $D^{-}$and $D^{+}$, that is the subsets $g^{\frac{t}{2}} \partial_{+}^{1} D^{-}$and $g^{-\frac{t}{2}} \partial_{-}^{1} D^{+}$of $T^{1} \widetilde{M}$, intersect. Then their intersection is the singleton consisting of the tangent vector of $\alpha$ at its midpoint.

Lemma 7 For every $R>0$, there exist $t_{0}, c_{0}>0$ such that for all $\left.\left.\eta \in\right] 0,1\right]$ and all $t \in\left[t_{0},+\infty\left[\right.\right.$, for all nonempty closed convex subsets $D^{-}, D^{+}$in $\widetilde{M}$, and for all $w \in$ $g^{t / 2} \mathscr{V}_{\eta, R}^{+}\left(\partial_{+}^{1} D^{-}\right) \cap g^{-t / 2} \mathscr{V}_{\eta, R}^{-}\left(\partial_{-}^{1} D^{+}\right)$, there exist $\left.s \in\right]-2 \eta, 2 \eta[$ and a common perpendicular $\widetilde{c}$ from $D^{-}$to $D^{+}$such that

- the length of $\widetilde{c}$ is contained in $\left[t+s-c_{0} e^{-\frac{t}{2}}, t+s+c_{0} e^{-\frac{t}{2}}\right]$,

- if $w^{\mp}=f_{D^{\mp}}^{ \pm}(w)$ and if $p^{ \pm}$is the endpoint of $\widetilde{c}$ in $D^{ \pm}$, then $d\left(\pi\left(w^{ \pm}\right), p^{ \pm}\right) \leq c_{0} e^{-\frac{t}{2}}$,

- the basepoint $\pi(w)$ of $w$ is at distance at most $c_{0} e^{-\frac{t}{2}}$ from a point of $\widetilde{c}$, and

$$
\begin{aligned}
& \max \left\{d\left(\pi\left(g^{\frac{t}{2}} w^{-}\right), \pi(w)\right), d\left(\pi\left(g^{-\frac{t}{2}} w^{+}\right), \pi(w)\right)\right\} \leq \eta+c_{0} e^{-\frac{t}{2}} . \\
& g^{-\frac{t}{2}-s^{-}} w \\
& \text { y w } \\
& x^{-} \quad \alpha^{-w^{-}} \\
& \iota w^{+} \alpha^{+} \quad x^{+} w^{+} \\
& y^{\prime} \\
& p^{-} \\
& D^{-} \\
& y^{\prime \prime} \\
& \widetilde{c} \\
& p^{+} \\
& D^{+}
\end{aligned}
$$

Proof. Let $t \geq 3$ and $\eta \in] 0,1]$. By definition of the dynamical neighbourhoods $\mathscr{V}_{\eta, R}^{\mp}\left(\partial_{\mp}^{1} D^{ \pm}\right)$, there exist $w^{ \pm} \in \partial_{\mp}^{1} D^{ \pm}$and $\left.s^{ \pm} \in\right]-\eta,+\eta[$ such that

$$
d_{W^{+}\left(w^{-}\right)}\left(g^{-\frac{t}{2}-s^{-}} w, w^{-}\right) \leq R \quad \text { and } \quad d_{W^{-}\left(w^{+}\right)}\left(g^{\frac{t}{2}+s^{+}} w, w^{+}\right) \leq R .
$$

Let $x^{ \pm}=\pi\left(w^{ \pm}\right), y=\pi(w)$, and let $\alpha^{-}$(respectively $\alpha^{+}$) be the angle at $x^{-}$(respectively $x^{+}$) between $w^{-}$(respectively $\iota w^{+}$) and the geodesic segment $\left[x^{-}, x^{+}\right]$.

Step 1. Let $\bar{\alpha}^{-}$(respectively $\bar{\alpha}^{+}$) be the angle at $x^{-}$(respectively $x^{+}$) between the outer normal vector $w^{-}$(respectively $\iota w^{+}$) and the geodesic segment $\left[x^{-}, y\right]$ (respectively $\left[x^{+}, y\right]$ ). Let $\beta^{ \pm}$be the angle at $y$ between $\pm w$ and the geodesic segment $\left[y, x^{ \pm}\right]$. Let us prove that there exist two constants $t_{1}, c_{1}>0$ depending only on $R$ such that if $t \geq t_{1}$ then $\bar{\alpha}^{ \pm}, \beta^{ \pm} \leq c_{1} e^{-\frac{t}{2}}$.

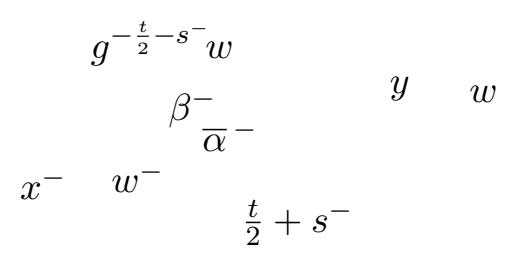

By Lemma 5 and Equation (2), we have

$$
d\left(\pi\left(g^{-\frac{t}{2}-s^{-}} w\right), x^{-}\right) \leq d_{W^{+}\left(w^{-}\right)}\left(g^{-\frac{t}{2}-s^{-}} w, w^{-}\right) \leq R,
$$




$$
d\left(y, \pi\left(g^{\frac{t}{2}+s^{-}} w^{-}\right)\right) \leq d_{W^{+}(w)}\left(w, g^{\frac{t}{2}+s^{-}} w^{-}\right) \leq R e^{-\frac{t}{2}-s^{-}} .
$$

In particular,

$$
d\left(\pi(w), \pi\left(g^{\frac{t}{2}} w^{-}\right)\right) \leq d\left(y, \pi\left(g^{\frac{t}{2}+s^{-}} w^{-}\right)\right)+\left|s^{-}\right| \leq R e^{-\frac{t}{2}-s^{-}}+\eta \leq c_{0} e^{-\frac{t}{2}}+\eta
$$

if we assume, as we may, that $c_{0} \geq R e^{\eta}$. With a similar argument for $w^{+}$, this proves the last formula of Lemma 7 .

Recall that by a hyperbolic trigonometric formula (see for instance [Bea, p. 147]), for any geodesic triangle in the real hyperbolic plane, with angles $\alpha, \beta, \gamma$ and opposite side lengths $a, b, c$, if $\gamma \geq \frac{\pi}{2}$, then $\tan \alpha \leq \frac{1}{\sinh b}$, which is at most $\frac{1}{\sinh (c-a)}$ if $c>a$ by the triangle inequality. By comparison, if $t \geq 2(R+2)$ (which implies that $\frac{t}{2}+s^{-}-R \geq 1$ ), we hence have

$$
\max \left\{\tan \bar{\alpha}^{-}, \tan \beta^{-}\right\} \leq \frac{1}{\sinh \left(\frac{t}{2}+s^{-}-R\right)} \leq 4 e^{-\frac{t}{2}-s^{-}+R}
$$

With a symmetric argument for $\bar{\alpha}^{+}, \beta^{+}$, the result follows.

Step 2. Let $\overline{\bar{\alpha}}^{ \pm}$be the angles at $x^{ \pm}$of the geodesic triangle with vertices $x_{-}, x_{+}, y$. Let $y^{\prime}$ be the closest point to $y$ on the side $\left[x_{-}, x_{+}\right]$. Let us prove that there exist two constants $t_{2}, c_{2}>0 \quad x^{-}$ depending only on $R$ such that if $t \geq t_{2}$ then $\overline{\bar{\alpha}}^{ \pm}, d\left(y, y^{\prime}\right) \leq c_{2} e^{-\frac{t}{2}}$.

Since the angle $\angle_{y}\left(x^{-}, x^{+}\right)$is at least $\pi-\beta^{-}-\beta^{+}$, at least one of the two angles $\angle_{y}\left(y^{\prime}, x^{ \pm}\right)$is at least $\frac{\pi-\beta^{-}-\beta^{+}}{2}$. By a comparison argument applied to one of the two triangles with vertices $\left(y, y^{\prime}, x^{ \pm}\right)$, as in the end of the first step, we have $\tan \frac{\pi-\beta^{-}-\beta^{+}}{2} \leq$ $\frac{1}{\sinh d\left(y, y^{\prime}\right)}$. Hence

$$
d\left(y, y^{\prime}\right) \leq \sinh d\left(y, y^{\prime}\right) \leq \tan \frac{\beta^{-}+\beta^{+}}{2},
$$

and the desired majoration of $d\left(y, y^{\prime}\right)$ follows from Step 1. By the same argument, we have $\tan \overline{\bar{\alpha}}^{ \pm} \leq \frac{1}{\sinh \left(d\left(x^{ \pm}, y\right)-d\left(y, y^{\prime}\right)\right)}$. Since $d\left(x^{ \pm}, y\right) \geq \frac{t}{2}-s^{ \pm}-R e^{-\frac{t}{2}-s^{-}}$by the inverse triangle inequality and Equation (7), the desired majoration of $\overline{\bar{\alpha}}^{ \pm}$follows.

Step 3. Let us prove that there exist two constants $t_{3}, c_{3}>0$ depending only on $R$ such that if $t \geq t_{3}$ then there exists a common perpendicular $\widetilde{c}=\left[p^{-}, p^{+}\right]$from $D^{-}$to $D^{+}$ such that $d\left(x^{-}, p^{-}\right), d\left(x^{+}, p^{+}\right) \leq c_{3} e^{-\frac{t}{2}}$. This will prove the second point of Lemma 7 (if $t_{0} \geq t_{3}$ and $\left.c_{0} \geq c_{3}\right)$.

By the first two steps, we have, if $t \geq \min \left\{t_{1}, t_{2}\right\}$,

$$
\alpha^{ \pm} \leq \bar{\alpha}^{ \pm}+\overline{\bar{\alpha}}^{ \pm} \leq\left(c_{1}+c_{2}\right) e^{-\frac{t}{2}} .
$$

Assume by absurd that the intersection of the closures of $D^{-}$and $D^{+}$in $\widetilde{M} \cup \partial_{\infty} \widetilde{M}$ contains a point $z$. Then by convexity of $D^{ \pm}$, and since the distance $d\left(x^{-}, x^{+}\right)$is large and the angles $\alpha^{ \pm}$are small if $t$ is large, the angles at $x^{ \pm}$of the geodesic triangle with vertices $z, x^{-}, x^{+}$are almost at least $\frac{\pi}{2}$, which is impossible since $\widetilde{M}$ is $\operatorname{CAT}(-1)$. Hence the nonempty closed convex subsets $D^{-}$and $D^{+}$have a common perpendicular $\widetilde{c}=\left[p^{-}, p^{+}\right]$, with $p^{ \pm} \in D^{ \pm}$. 
Consider the geodesic quadrilateral $Q$ with vertices $x^{ \pm}, p^{ \pm}$. By convexity of $D^{ \pm}$, its angles at $p^{ \pm}$are at least $\frac{\pi}{2}$ and its angles at $x^{ \pm}$are at least $\frac{\pi}{2}-\alpha^{ \pm}$. Note that if $t \geq t_{2}^{\prime}=2\left(R+c_{2}+1+\operatorname{argsinh} 2\right)$ then we have, by Step 2 ,

$$
\begin{aligned}
d\left(x^{-}, x^{+}\right) & \geq d\left(x^{-}, y\right)+d\left(y, x^{+}\right)-2 d\left(y, y^{\prime}\right) \\
& \geq\left(\frac{t}{2}+s^{-}-R\right)+\left(\frac{t}{2}+s^{+}-R\right)-2 c_{2} e^{-\frac{t}{2}} \geq 2 \operatorname{argsinh} 2 .
\end{aligned}
$$

Up to replacing $Q$ by a comparison quadrilateral (obtained by gluing two comparison triangles) in the real hyperbolic plane $\mathbb{H}_{\mathbb{R}}^{2}$, having the same side lengths and bigger angles, we may assume that $\widetilde{M}=\mathbb{H}_{\mathbb{R}}^{2}$ and that $x^{-}$and $x^{+}$are on the same side of the geodesic line through $p^{-}, p^{+}$. Up to replacing $Q$ by a quadrilateral having same distances $d\left(x^{-}, x^{+}\right)$, $d\left(x^{-}, p^{-}\right), d\left(x^{+}, p^{+}\right)$and bigger angles at $x^{-}, x^{+}$, we may assume that the angles at $p^{-}, p^{+}$ are exactly $\frac{\pi}{2}$. If, say, the angle at $x^{+}$was bigger than the angle at $x^{-}$, up to replacing $x^{+}$ by a point on the geodesic line through $p^{+}, x^{+}$on the other side of $x^{+}$than $p^{+}$if $p^{+} \neq x^{+}$, which increases $d\left(x^{-}, x^{+}\right), d\left(x^{+}, p^{+}\right)$, decreases the angle at $x^{+}$and increases the angle at $x^{-}$, we may assume that the angles at $x^{ \pm}$are equal, and we denote this common value by $\phi \geq \frac{\pi}{2}-\min \left\{\alpha^{-}, \alpha^{+}\right\}$.

Let $b_{1}=\frac{1}{2} d\left(x^{-}, x^{+}\right)$and $b_{2}=d\left(x^{-}, p^{-}\right)=$ $d\left(x^{+}, p^{+}\right)$. By formulas of [Bea, p. 157] on Lambert quadrilaterals, we have

$$
\cosh b_{2}=\frac{\sinh b_{1}}{\sqrt{\sinh ^{2} b_{1} \sin ^{2} \phi-\cos ^{2} \phi}} .
$$

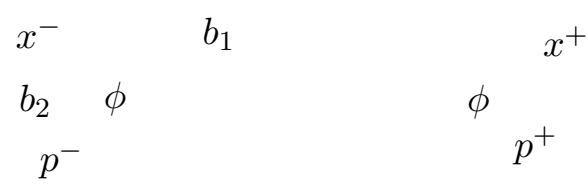

By Equation (8), with $c_{2}^{\prime}=c_{1}+c_{2}$, let $t_{2}^{\prime \prime}>0$ be a constant, depending only on $R$, such that if $t \geq t_{2}^{\prime \prime}$, then $\sin \phi \geq \max \left\{\cos \alpha^{ \pm}\right\} \geq 1-c_{2}^{\prime 2} e^{-t} \geq 1 / 2$. By Equation (9), if $t \geq t_{2}^{\prime}$, then $b_{1} \geq \frac{t}{2}-R-1-c_{2} \geq \operatorname{argsinh} 2$ (and in particular $1 / \sinh b_{1} \leq 1 / 2$ ). Hence, if $\left.t \geq \max \left\{t_{2}^{\prime}, t_{2}^{\prime \prime}\right)\right\}$, then

$\cosh b_{2} \leq \frac{1}{\sqrt{\sin ^{2} \phi-\frac{1}{\sinh ^{2} b_{1}}}} \leq\left(\left(1-c_{2}^{\prime 2} e^{-t}\right)^{2}-\frac{1}{\sinh ^{2}\left(\frac{t}{2}-R-1-c_{2}\right)}\right)^{-\frac{1}{2}}=1+\mathrm{O}\left(e^{-t}\right)$.

Since $\cosh u \sim 1+\frac{u^{2}}{2}$ as $u \rightarrow 0$, Step 3 follows.

Step 4: Conclusion. Let $t \geq t_{0}=\max \left\{t_{2}, t_{3}, 3\right\}, c_{0}=\max \left\{2 e^{2} R, 2\left(c_{2}+c_{3}\right)\right\}$ and, with the previous notation, let $\left.s=s^{-}+s^{+} \in\right]-2 \eta, 2 \eta[$. By convexity, the triangle inequality and Equation (7), we have

$$
\begin{aligned}
d\left(p^{-}, p^{+}\right) & \leq d\left(x^{-}, x^{+}\right) \leq d\left(x^{-}, y\right)+d\left(y, x^{+}\right) \\
& \leq\left(\frac{t}{2}+s^{-}+R e^{-\frac{t}{2}-s^{-}}\right)+\left(\frac{t}{2}+s^{+}+R e^{-\frac{t}{2}-s^{+}}\right) \leq t+s+c_{0} e^{-\frac{t}{2}} .
\end{aligned}
$$

Similarly, using Step 3 and Step 2, we have

$$
\begin{aligned}
d\left(p^{-}, p^{+}\right) & \geq d\left(x^{-}, x^{+}\right)-d\left(p^{-}, x^{-}\right)-d\left(x^{+}, p^{+}\right) \geq d\left(x^{-}, x^{+}\right)-2 c_{3} e^{-\frac{t}{2}} \\
& \geq d\left(x^{-}, y\right)+d\left(y, x^{+}\right)-2 d\left(y, y^{\prime}\right)-2 c_{3} e^{-\frac{t}{2}} \\
& \geq\left(\frac{t}{2}+s^{-}\right)+\left(\frac{t}{2}+s^{+}\right)-2\left(c_{2}+c_{3}\right) e^{-\frac{t}{2}} \geq t+s-c_{0} e^{-\frac{t}{2}} .
\end{aligned}
$$


Let $y^{\prime \prime}$ be the closest point to $y^{\prime}$ on the common perpendicular $\left[p^{-}, p^{+}\right]$(see the picture before this proof). Then, by Step 2, and by convexity and Step 3, we have

$$
d\left(y, y^{\prime \prime}\right) \leq d\left(y, y^{\prime}\right)+d\left(y^{\prime}, y^{\prime \prime}\right) \leq c_{2} e^{-\frac{t}{2}}+c_{3} e^{-\frac{t}{2}} \leq c_{0} e^{-\frac{t}{2}} .
$$

This concludes the proof of Lemma 7 .

\section{Counting and equidistribution of common perpendiculars}

Let $\widetilde{M}, x_{0}, \Gamma, \delta$ and $M$ be as in the beginning of Section 2 .

\subsection{A reminder on Patterson-Sullivan and skinning measures}

A family $\left(\mu_{x}\right)_{x \in \widetilde{M}}$ of finite measures on $\partial_{\infty} \widetilde{M}$, whose support is the limit set $\Lambda \Gamma$ of $\Gamma$, is a Patterson-Sullivan density for $\Gamma$ if

$$
\gamma_{*} \mu_{x}=\mu_{\gamma x}
$$

for all $\gamma \in \Gamma$ and $x \in \widetilde{M}$, and if the following Radon-Nikodym derivatives exist for all $x, y \in \widetilde{M}$ and satisfy for (almost) all $\xi \in \partial_{\infty} \widetilde{M}$

$$
\frac{d \mu_{x}}{d \mu_{y}}(\xi)=e^{-\delta \beta_{\xi}(x, y)} .
$$

We fix such a family $\left(\mu_{x}\right)_{x \in \widetilde{M}}$. The Bowen-Margulis measure on $T^{1} \widetilde{M}$ (associated with this Patterson-Sullivan density) is the measure $\widetilde{m}_{\mathrm{BM}}$ on $T^{1} \widetilde{M}$ given by the density

$$
d \widetilde{m}_{\mathrm{BM}}(v)=e^{-\delta\left(\beta_{v_{-}}\left(\pi(v), x_{0}\right)+\beta_{v_{+}}\left(\pi(v), x_{0}\right)\right)} d \mu_{x_{0}}\left(v_{-}\right) d \mu_{x_{0}}\left(v_{+}\right) d t
$$

in Hopf's parametrisation. The Bowen-Margulis measure $\widetilde{m}_{\mathrm{BM}}$ is independent of $x_{0}$, and it is invariant under the actions of the group $\Gamma$ and of the geodesic flow. Thus, it defines a measure $m_{\mathrm{BM}}$ on $T^{1} M$ which is invariant under the quotient geodesic flow, called the Bowen-Margulis measure on $T^{1} M$. If $m_{\mathrm{BM}}$ is finite, then the Patterson-Sullivan densities are unique up to a multiplicative constant; hence the Bowen-Margulis measure is uniquely defined, up to a multiplicative constant. When finite and normalised to be a probability measure, it is the unique measure of maximal entropy of the geodesic flow, if the sectional curvature of $\widetilde{M}$ has bounded derivatives.

Babillot [Bab, Thm. 1] showed that if the Bowen-Margulis measure is finite, then it is mixing for the geodesic flow of $M$ if the length spectrum of $M$ is not contained in a discrete subgroup of $\mathbb{R}$. This condition is satisfied, for example, if $\Gamma$ has a parabolic element, if $\Lambda \Gamma$ is not totally disconnected (hence if $M$ is compact), or if $\widetilde{M}$ is a surface or a (rank-one) symmetric space, see for instance [Dal1, Dal2].

We refer to [DOP] for finiteness criteria of $m_{\mathrm{BM}}$. In particular, if $\Gamma$ is geometrically finite (see for instance [Bow for a definition), if the critical exponent of the stabiliser $\Gamma_{p}$ of any parabolic fixed point $p$ in $\Gamma$ is strictly smaller than $\delta$, then $m_{\mathrm{BM}}$ is finite.

Let $D$ be a nonempty proper closed convex subset of $\widetilde{M}$. The (outer) skinning measure on $\partial_{+}^{1} D$ (associated with the Patterson-Sullivan density $\left(\mu_{x}\right)_{x \in \widetilde{M}}$ ) is the measure $\widetilde{\sigma}_{D}^{+}$on 
$\partial_{+}^{1} D$ defined, using the positive endpoint homeomorphism $v \mapsto v_{+}$from $\partial_{+}^{1} D$ to $\partial_{\infty} \widetilde{M}-$ $\partial_{\infty} D$, by

$$
d \widetilde{\sigma}_{D}^{+}(v)=e^{-\delta \beta_{v_{+}}\left(P_{D}\left(v_{+}\right), x_{0}\right)} d \mu_{x_{0}}\left(v_{+}\right),
$$

and the (inner) skinning measure on $\partial_{-}^{1} D=\iota \partial_{+}^{1} D$ is the measure $\widetilde{\sigma}_{D}^{-}=\iota_{*} \widetilde{\sigma}_{D}^{+}$. Since $P_{D}\left(v_{ \pm}\right)=\pi(v)$ for every $v \in \partial_{ \pm}^{1} D$, we will often replace $P_{D}\left(v_{ \pm}\right)$by $\pi(v)$ in the above formulas when there is no doubt on what $v$ is. We refer to PP5] for more background and for the basic properties of these measures.

The skinning measures associated with horoballs are of particular importance in this paper. Let $w \in T^{1} \widetilde{M}$. We denote the skinning measures on the strong stable and strong unstable leaves $W^{+}(w)$ and $W^{-}(w)$ of $w$ by

$$
\mu_{W^{+}(w)}=\widetilde{\sigma}_{H B_{+}(w)}^{-} \quad \text { and } \quad \mu_{W^{-}(w)}=\tilde{\sigma}_{H B_{-}(w)}^{+} .
$$

\subsection{Equidistribution of endvectors of common perpendiculars in $T^{1} \widetilde{M}$}

Let $I$ be an index set endowed with a left action $(\gamma, i) \mapsto \gamma i$ of $\Gamma$. A family $\mathscr{D}=\left(D_{i}\right)_{i \in I}$ of subsets of $\widetilde{M}$ or $T^{1} \widetilde{M}$ indexed by $I$ is $\Gamma$-equivariant if $\gamma D_{i}=D_{\gamma i}$ for all $\gamma \in \Gamma$ and $i \in I$. We equip the index set $I$ with the $\Gamma$-equivariant equivalence relation $\sim$ defined by $i \sim j$ if and only if there exists $\gamma \in \operatorname{Stab}_{\Gamma} D_{i}$ such that $j=\gamma i$ (or equivalently if $D_{j}=D_{i}$ and $j=\gamma i$ for some $\gamma \in \Gamma$ ). Note that $\Gamma$ acts on the left on the set of equivalence classes $I / \sim$.

An example of such a family is given by fixing a subset $D$ of $\widetilde{M}$ or $T^{1} \widetilde{M}$, by setting $I=\Gamma$ with the left action by translations $(\gamma, i) \mapsto \gamma i$, and by setting $D_{i}=i D$ for every $i \in \Gamma$. In this case, we have $i \sim j$ if and only if $i^{-1} j$ belongs to the stabiliser $\Gamma_{D}$ of $D$ in $\Gamma$, and $I / \sim=\Gamma / \Gamma_{D}$. More general examples include $\Gamma$-orbits of (usually finite) collections of subsets of $\widetilde{M}$ or $T^{1} \widetilde{M}$ with (usually finite) multiplicities.

A $\Gamma$-equivariant family $\left(A_{i}\right)_{i \in I}$ of closed subsets of $\widetilde{M}$ or $T^{1} \widetilde{M}$ is locally finite if for every compact subset $K$ in $\widetilde{M}$ or $T^{1} \widetilde{M}$, the quotient set $\left\{i \in I: A_{i} \cap K \neq \emptyset\right\} / \sim$ is finite. In particular, the union of the images of the sets $A_{i}$ by the map $\widetilde{M} \rightarrow M$ or $T^{1} \widetilde{M} \rightarrow T^{1} M$ is closed. When $\Gamma \backslash I$ is finite, $\left(A_{i}\right)_{i \in I}$ is locally finite if and only if, for all $i \in I$, the canonical map from $\Gamma_{A_{i}} \backslash A_{i}$ to $M$ or $T^{1} M$ is proper, where $\Gamma_{A_{i}}$ is the stabiliser of $A_{i}$ in $\Gamma$.

Let $\mathscr{D}^{-}=\left(D_{i}^{-}\right)_{i \in I^{-}}$and $\mathscr{D}^{+}=\left(D_{j}^{+}\right)_{j \in I^{+}}$be locally finite $\Gamma$-equivariant families of nonempty proper closed convex subsets of $\widetilde{M}$. For every $(i, j)$ in $I^{-} \times I^{+}$such that $D_{i}^{-}$ and $D_{j}^{+}$have a common perpendicular, we denote by $\alpha_{i, j}$ this common perpendicular, by $\ell\left(\alpha_{i, j}\right)$ its length, by $v_{i, j}^{-} \in \partial_{+}^{1} D_{i}^{-}$its initial tangent vector and by $v_{i, j}^{+} \in \partial_{-}^{1} D_{i}^{+}$its terminal tangent vector. Note that if $i^{\prime} \sim i, j^{\prime} \sim j$ and $\gamma \in \Gamma$, then

$$
\gamma \alpha_{i^{\prime}, j^{\prime}}=\alpha_{\gamma i, \gamma j}, \quad \ell\left(\alpha_{i^{\prime}, j^{\prime}}\right)=\ell\left(\alpha_{\gamma i, \gamma j}\right) \quad \text { and } \quad \gamma v_{i^{\prime}, j^{\prime}}^{ \pm}=v_{\gamma i, \gamma j}^{ \pm}
$$

The inner and outer skinning measures of the families $\mathscr{D}^{ \pm}$on $T^{1} \widetilde{M}$ are

$$
\tilde{\sigma}_{\mathscr{D} \mp}^{ \pm}=\sum_{i \in I^{\mp} / \sim} \widetilde{\sigma}_{D_{i}}^{ \pm}
$$

We will now prove that the ordered pairs of initial and terminal tangent vectors of common perpendiculars of two locally finite equivariant families of convex sets in $\widetilde{M}$ equidistribute towards the product of the skinning measures of the families. 
Theorem 8 Let $\widetilde{M}$ be a complete simply connected Riemannian manifold with pinched sectional curvature at most -1 . Let $\Gamma$ be a nonelementary discrete group of isometries of $\widetilde{M}$. Let $\mathscr{D}^{-}=\left(D_{i}^{-}\right)_{i \in I^{-}}$and $\mathscr{D}^{+}=\left(D_{j}^{+}\right)_{j \in I^{+}}$be locally finite $\Gamma$-equivariant families of nonempty proper closed locally convex subsets of $\widetilde{M}$. Assume that the Bowen-Margulis measure $m_{\mathrm{BM}}$ is finite and mixing for the geodesic flow. Then

$$
\lim _{t \rightarrow+\infty} \delta\left\|m_{\mathrm{BM}}\right\| e^{-\delta t} \sum_{\substack{i \in I^{-} / \sim, j \in I^{+} / \sim, \gamma \in \Gamma \\ D_{i}^{-} \cap \gamma \overline{D_{j}^{+}}=\emptyset, \ell\left(\alpha_{i, \gamma j}\right) \leq t}} \Delta_{v_{i, \gamma j}^{-}} \otimes \Delta_{v_{\gamma^{-1} i, j}^{+}}=\tilde{\sigma}_{\mathscr{D}^{-}}^{+} \otimes \tilde{\sigma}_{\mathscr{D}^{+}}^{-}
$$

for the weak-star convergence of measures on the locally compact space $T^{1} \widetilde{M} \times T^{1} \widetilde{M}$.

If $\mathscr{D}^{-}=(\gamma x)_{\gamma \in \Gamma}$ and $\mathscr{D}^{+}=(\gamma y)_{\gamma \in \Gamma}$ for some $x, y \in \widetilde{M}$, this statement is a consequence of the proof of [Rob, Theo. 4.1.1]. We use the same technical initial trick as Roblin but immediately after that we use a functional approach, better suited to obtain error terms in Section 4. We will give a reformulation in $T^{1} M \times T^{1} M$ of this result in Section 3.3, and some applications to particular geometric situations in Section 6 .

Proof. We first give a scheme of the proof (see [PP8, §8] for a more elaborate one). The crucial observation is that two convex subsets $D^{-}$and $D^{+}$have a common perpendicular of length $t>0$ if and only if $g^{\frac{t}{2}} \partial_{+}^{1} D^{-}$and $g^{-\frac{t}{2}} \partial_{-}^{1} D^{+}$intersect. After a reduction of the statement, we introduce test functions $\phi_{\eta}^{\mp}$ vanishing outside a small dynamical neighbourhood of $\partial_{ \pm}^{1} D^{\mp}$, so that the support of the product function $\phi_{\eta}^{-} \circ g^{-\frac{t}{2}} \phi_{\eta}^{+} \circ g^{\frac{t}{2}}$ detects the intersection of $g^{\frac{t}{2}} \partial_{+}^{1} D^{-}$and $g^{-\frac{t}{2}} \partial_{-}^{1} D^{+}$(using Subsection 2.3). We will then use the mixing of the geodesic flow to obtain the equidistribution result.

The estimation of the small terms occuring in the following steps 2, 4 and 5 is much more precise than what is needed to prove Theorem 8 . These estimates will be useful to give a speed of equidistribution of the initial and terminal vectors, and an error term in the asymptotic of the counting function $\mathscr{N}_{\mathscr{D}^{-}, \mathscr{D}^{+}}(t)$ in Theorem 15

To shorten the notation, we assume from now on that the sums as in the statement of Theorem 8 are for $(i, j, \gamma)$ such that $\alpha_{i, \gamma j}$ exists, that is $\overline{D_{i}^{-}} \cap \overline{\gamma D_{j}^{+}}=\emptyset$. By Equation (11), this sum is independent of the choice of representatives of $i$ in $I^{-} / \sim$ and $j$ in $I^{+} / \sim$.

Step 1: Reduction of the statement. By additivity, by the local finiteness of the families $\mathscr{D}^{ \pm}$, and by the definition of $\widetilde{\sigma}_{\mathscr{D} \mp}^{ \pm}=\sum_{k \in I^{\mp} / \sim} \widetilde{\sigma}_{D_{k}^{\mp}}^{ \pm}$, we only have to prove, for all fixed $i \in I^{-}$and $j \in I^{+}$, that, for the weak-star convergence of measures on $T^{1} \widetilde{M} \times T^{1} \widetilde{M}$,

$$
\lim _{t \rightarrow+\infty} \delta\left\|m_{\mathrm{BM}}\right\| e^{-\delta t} \sum_{\gamma \in \Gamma: 0<\ell\left(\alpha_{i, \gamma j}\right) \leq t} \Delta_{v_{i, \gamma j}^{-}} \otimes \Delta_{v_{\gamma^{-1}, j}^{+}}=\tilde{\sigma}_{D_{i}^{-}}^{+} \otimes \widetilde{\sigma}_{D_{j}^{+}}^{-}
$$

Let $\Omega^{-}$be a Borel subset of $\partial_{+}^{1} D_{i}^{-}$and let $\Omega^{+}$be a Borel subset of $\partial_{-}^{1} D_{j}^{+}$. To simplify the notation, let

$$
D^{-}=D_{i}^{-}, \quad D^{+}=D_{j}^{+}, \quad \alpha_{\gamma}=\alpha_{i, \gamma j}, \quad \ell_{\gamma}=\ell\left(\alpha_{\gamma}\right), \quad v_{\gamma}^{ \pm}=v_{i, \gamma j}^{ \pm} \text {and } \tilde{\sigma}^{ \pm}=\tilde{\sigma}_{D^{\mp}}^{ \pm} .
$$

Let $v_{\gamma}^{0}$ be the tangent vector at the midpoint of $\alpha_{\gamma}$ (see the picture below, sitting in $T^{1} \widetilde{M}$ ). 


$$
\begin{aligned}
& \partial_{+}^{1} D^{-} \quad g^{\ell_{\gamma} / 2} \partial_{+}^{1} D^{-} \\
& W^{\mathrm{ss}}\left(v_{\gamma}^{-}\right) \quad W^{\mathrm{ss}}\left(v_{\gamma}^{0}\right) \\
& v_{\gamma}^{-} \quad v_{\gamma}^{0} \quad v_{\gamma}^{+} \\
& g^{-\ell_{\gamma} / 2} \gamma \partial_{-}^{1} D^{+} \\
& \gamma \partial_{-}^{1} D^{+} \\
& W^{\mathrm{su}}\left(v_{\gamma}^{0}\right) \quad W^{\mathrm{su}}\left(v_{\gamma}^{+}\right)
\end{aligned}
$$

Assume that $\Omega^{-}$and $\Omega^{+}$have positive finite skinning measures (for future use, we do not assume them to be relatively compact), and that their boundaries in $\partial_{+}^{1} D^{-}$and $\partial_{-}^{1} D^{+}$ have zero skinning measures. Let

$$
I_{\Omega^{-}, \Omega^{+}}(t)=\delta\left\|m_{\mathrm{BM}}\right\| e^{-\delta t} \operatorname{Card}\left\{\gamma \in \Gamma: 0<\ell_{\gamma} \leq t, v_{\gamma}^{-} \in \Omega^{-}, v_{\gamma}^{+} \in \gamma \Omega^{+}\right\} .
$$

Let us prove the stronger statement that, for every such $\Omega^{ \pm}$, we have

$$
\lim _{t \rightarrow+\infty} I_{\Omega^{-}, \Omega^{+}}(t)=\tilde{\sigma}^{+}\left(\Omega^{-}\right) \tilde{\sigma}^{-}\left(\Omega^{+}\right) .
$$

Step 2: Construction of the bump functions. We recall from [PP5, §5] the definition of the test functions $\phi_{\eta}^{ \pm}$. We fix $R>0$ such that $\mu_{W^{ \pm}(w)}\left(B^{ \pm}(w, R)\right)>0$ for all $w \in \partial_{\mp}^{1} D^{ \pm}$, hence for all $w \in \gamma \partial_{\mp}^{1} D^{ \pm}$with $\gamma \in \Gamma$. Such an $R$ exists by [PP5, Lem. 7]. For all $\eta, \eta^{\prime}>0$, let $h_{\eta, \eta^{\prime}}^{ \pm}: T^{1} \widetilde{M} \rightarrow[0,+\infty[$ be the $\Gamma$-invariant measurable maps defined by

$$
h_{\eta, \eta^{\prime}}^{\mp}(w)=\frac{1}{2 \eta \mu_{W^{ \pm}(w)}\left(B^{ \pm}\left(w, \eta^{\prime}\right)\right)}
$$

if $\mu_{W^{ \pm}(w)}\left(B^{ \pm}\left(w, \eta^{\prime}\right)\right)>0$ (which is satisfied if $w_{ \pm} \in \Lambda \Gamma$ ), and $h_{\eta, \eta^{\prime}}^{ \pm}(w)=0$ otherwise.

Let us denote by $\mathbb{1}_{A}$ the characteristic function of a subset $A$. We define the test functions $\phi_{\eta}^{\mp}=\phi_{\eta, R, \Omega^{ \pm}}^{\mp}: T^{1} \widetilde{M} \rightarrow[0,+\infty[$ by

$$
\phi_{\eta}^{\mp}=h_{\eta, R}^{\mp} \circ f_{D^{\mp}}^{ \pm} \mathbb{1}_{\mathscr{V}_{\eta, R}^{ \pm}\left(\Omega^{\mp}\right)},
$$

where $\mathscr{V}_{\eta, R}^{ \pm}\left(\Omega^{\mp}\right)$ and $f_{D^{\mp}}^{ \pm}$are as in Subsection 2.2 . Note that $v$ belongs to the domain of definition of $f_{D^{\mp}}^{ \pm}$if $v \in \mathscr{V}_{\eta, R}^{ \pm}\left(\Omega^{\mp}\right)$, otherwise $\phi_{\eta}^{\mp}(v)=0$. For all $v \in T^{1} \widetilde{M}$ and $t \geq 0$, we have, by [PP5, Lem. 17],

$$
\phi_{\eta, R, \Omega^{+}}^{-}\left(g^{-t} v\right)=e^{-\delta t} \phi_{\eta, e^{-t} R, g^{t} \Omega^{+}}^{-}(v) .
$$

Now, the heart of the proof is to give two pairs of upper and lower bounds, as $T \geq 0$ is large enough and $\eta \in] 0,1]$ is small enough, of the quantity

$$
i_{\eta}(T)=\int_{0}^{T} e^{\delta t} \sum_{\gamma \in \Gamma} \int_{T^{1} \widetilde{M}}\left(\phi_{\eta}^{-} \circ g^{-t / 2}\right)\left(\phi_{\eta}^{+} \circ g^{t / 2} \circ \gamma^{-1}\right) d \widetilde{m}_{\mathrm{BM}} d t .
$$


Step 3: First upper and lower bounds. For all $t \geq 0$, let

$$
a_{\eta}(t)=\sum_{\gamma \in \Gamma} \int_{v \in T^{1} \widetilde{M}} \phi_{\eta}^{-}\left(g^{-t / 2} v\right) \phi_{\eta}^{+}\left(g^{t / 2} \gamma^{-1} v\right) d \widetilde{m}_{\mathrm{BM}}(v) .
$$

Note that by [PP5, Prop. 18], we have $\int_{T^{1}} \widetilde{M}_{\eta} \phi_{\eta}^{\mp} d \widetilde{m}_{\mathrm{BM}}=\widetilde{\sigma}^{ \pm}\left(\Omega^{\mp}\right)$, which is finite and positive. By passing to the universal cover the mixing property of the geodesic flow on $T^{1} M$, for every $\epsilon>0$, there hence exists $T_{\epsilon} \geq 0$ such that for all $t \geq T_{\epsilon}$, we have

$\frac{e^{-\epsilon}}{\left\|m_{\mathrm{BM}}\right\|} \int_{T^{1} \widetilde{M}} \phi_{\eta}^{-} d \widetilde{m}_{\mathrm{BM}} \int_{T^{1} \widetilde{M}} \phi_{\eta}^{+} d \widetilde{m}_{\mathrm{BM}} \leq a_{\eta}(t) \leq \frac{e^{\epsilon}}{\left\|m_{\mathrm{BM}}\right\|} \int_{T^{1} \widetilde{M}} \phi_{\eta}^{-} d \widetilde{m}_{\mathrm{BM}} \int_{T^{1} \widetilde{M}} \phi_{\eta}^{+} d \widetilde{m}_{\mathrm{BM}}$.

Hence for every $\epsilon>0$, there exists $c_{\epsilon, \eta}>0$ such that for all $T \geq 0$, we have

$$
e^{-\epsilon} \frac{e^{\delta T}}{\delta\left\|m_{\mathrm{BM}}\right\|} \widetilde{\sigma}^{+}\left(\Omega^{-}\right) \widetilde{\sigma}^{-}\left(\Omega^{+}\right)-c_{\epsilon, \eta} \leq i_{\eta}(T) \leq e^{\epsilon} \frac{e^{\delta T}}{\delta\left\|m_{\mathrm{BM}}\right\|} \widetilde{\sigma}^{+}\left(\Omega^{-}\right) \widetilde{\sigma}^{-}\left(\Omega^{+}\right)+c_{\epsilon, \eta} .
$$

Step 4: Second upper and lower bounds. Let $T \geq 0$ and $\eta \in] 0,1]$. By Fubini's theorem for nonnegative measurable maps and the definition of the test functions $\phi_{\eta}^{ \pm}$,

$$
\begin{array}{r}
i_{\eta}(T)=\sum_{\gamma \in \Gamma} \int_{0}^{T} e^{\delta t} \int_{T^{1} \widetilde{M}} h_{\eta, R}^{-} \circ f_{D^{-}}^{+}\left(g^{-t / 2} v\right) h_{\eta, R}^{+} \circ f_{D^{+}}^{-}\left(\gamma^{-1} g^{t / 2} v\right) \\
\mathbb{1}_{\mathscr{V}_{\eta, R}^{+}\left(\Omega^{-}\right)}\left(g^{-t / 2} v\right) \mathbb{1}_{\mathscr{V}_{\eta, R}^{-}\left(\Omega^{+}\right)}\left(\gamma^{-1} g^{t / 2} v\right) d \widetilde{m}_{\mathrm{BM}}(v) d t .
\end{array}
$$

We start the computations by rewriting the product term involving the technical maps $h_{\eta, R}^{ \pm}$. For all $\gamma \in \Gamma$ and $v \in \mathscr{U}_{D^{-}}^{+} \cap \mathscr{U}_{\gamma D^{+}}^{-}$, define (using Equation (3))

$$
w^{-}=f_{D^{-}}^{+}(v) \quad \text { and } \quad w^{+}=f_{\gamma D^{+}}^{-}(v)=\gamma f_{D^{+}}^{-}\left(\gamma^{-1} v\right) \text {. }
$$

By the invariance of $f_{D^{\mp}}^{ \pm}$by precomposition by the geodesic flow, $w^{\mp}$ is unchanged if $v$ is replaced by $g^{s} v$ for any $s \in \mathbb{R}$. A computation, using Equation (3) and the $\Gamma$-invariance of $h_{\eta, R}^{ \pm}$, see also [PP5, p. 1334], shows that

$$
\begin{gathered}
h_{\eta, R}^{-} \circ f_{D^{-}}^{+}\left(g^{-\frac{t}{2}} v\right)=e^{-\delta \frac{t}{2}} h_{\eta, e^{-t / 2} R}^{-}\left(g^{t / 2} w^{-}\right), \\
h_{\eta, R}^{+} \circ f_{D^{+}}^{-}\left(\gamma^{-1} g^{\frac{t}{2}} v\right)=e^{-\delta \frac{t}{2}} h_{\eta, e^{-t / 2}}^{+}\left(g^{-\frac{t}{2}} w^{+}\right),
\end{gathered}
$$

hence,

$$
h_{\eta, R}^{-} \circ f_{D^{-}}^{+}\left(g^{-t / 2} v\right) h_{\eta, R}^{+} \circ f_{D^{+}}^{-}\left(\gamma^{-1} g^{t / 2} v\right)=e^{-\delta t} h_{\eta, e^{-t / 2} R}^{-}\left(g^{t / 2} w^{-}\right) h_{\eta, e^{-t / 2} R}^{+}\left(g^{-t / 2} w^{+}\right) .
$$

The remaining product term $\mathbb{1}_{\mathscr{V}_{\eta, R}^{+}\left(\Omega^{-}\right)}\left(g^{-t / 2} v\right) \mathbb{1}_{\mathscr{V}_{\eta, R}^{-}\left(\Omega^{+}\right)}\left(\gamma^{-1} g^{t / 2} v\right)$ in Equation (19) is different from 0 (hence equal to 1 ) if and only if

$$
v \in g^{t / 2} \mathscr{V}_{\eta, R}^{+}\left(\Omega^{-}\right) \cap \gamma g^{-t / 2} \mathscr{V}_{\eta, R}^{-}\left(\Omega^{+}\right)=\mathscr{V}_{\eta, e^{-t / 2} R}^{+}\left(g^{t / 2} \Omega^{-}\right) \cap \mathscr{V}_{\eta, e^{-t / 2} R}^{-}\left(\gamma g^{-t / 2} \Omega^{+}\right),
$$


see Section 2.2, in particular Equation (6). By Lemma 7, there exists $t_{0}, c_{0}>0$ such that for all $\eta \in] 0,1]$ and $t \geq t_{0}$, for all $v \in T^{1} M$, if $\mathbb{1}_{\mathscr{V}_{\eta, R}^{+}\left(\Omega^{-}\right)}\left(g^{-t / 2} v\right) \mathbb{1}_{\mathscr{V}_{\eta, R}^{-}\left(\Omega^{+}\right)}\left(\gamma^{-1} g^{t / 2} v\right) \neq 0$, then the following facts hold:

(i) by the convexity of $D^{ \pm}$, we have $v \in \mathscr{U}_{D^{-}}^{+} \cap \mathscr{U}_{\gamma D^{+}}^{-}$,

(ii) by the definition of $w^{ \pm}$(see Equation (20)), we have $w^{-} \in \Omega^{-}$and $w^{+} \in \gamma \Omega^{+}$(The notation $\left(w^{-}, w^{+}\right)$here coincides with the notation $\left(w^{-}, w^{+}\right)$in Lemma 7),

(iii) there exists a common perpendicular $\alpha_{\gamma}$ from $D^{-}$to $\gamma D^{+}$with $\left|\ell_{\gamma}-t\right| \leq 2 \eta+c_{0} e^{-t / 2}$, $d\left(\pi\left(v_{\gamma}^{ \pm}\right), \pi\left(w^{ \pm}\right)\right) \leq c_{0} e^{-t / 2}, d\left(\pi\left(g^{ \pm t / 2} w^{\mp}\right), \pi(v)\right) \leq \eta+c_{0} e^{-t / 2}$ and such that $\pi(v)$ is at distance at most $c_{0} e^{-t / 2}$ from some point $p_{v}$ of $\alpha_{\gamma}$.

For all $\eta \in] 0,1], \gamma \in \Gamma$ and $T \geq t_{0}$, define

$$
\mathscr{A}_{\eta, \gamma}(T)=\left\{(t, v) \in\left[t_{0}, T\right] \times T^{1} \widetilde{M}: v \in \mathscr{V}_{\eta, e^{-t / 2} R}^{+}\left(g^{t / 2} \Omega^{-}\right) \cap \mathscr{V}_{\eta, e^{-t / 2} R}^{-}\left(\gamma g^{-t / 2} \Omega^{+}\right)\right\}
$$

and

$$
\begin{aligned}
& j_{\eta, \gamma}(T)=\iint_{(t, v) \in \mathscr{A}_{\eta, \gamma}(T)} h_{\eta, e^{-t / 2} R}^{-}\left(g^{t / 2} w^{-}\right) h_{\eta, e^{-t / 2} R}^{+}\left(g^{-t / 2} w^{+}\right) d t d \widetilde{m}_{\mathrm{BM}}(v) \\
& =\frac{1}{(2 \eta)^{2}} \iint_{(t, v) \in \mathscr{A}_{\eta, \gamma}(T)} \frac{d t d \widetilde{m}_{\mathrm{BM}}(v)}{\mu_{W^{+}\left(w_{t}^{-}\right)}\left(B^{+}\left(w_{t}^{-}, r_{t}\right)\right) \mu_{W^{-}\left(w_{t}^{+}\right)}\left(B^{-}\left(w_{t}^{+}, r_{t}\right)\right)} .
\end{aligned}
$$

with the notation

$$
r_{t}=e^{-t / 2} R, \quad w_{t}^{-}=g^{t / 2} w^{-} \text {and } w_{t}^{+}=g^{-t / 2} w^{+} .
$$

For all $s, r \in \mathbb{R}$, let $\Gamma_{s, r}=\left\{\gamma \in \Gamma: t_{0}+2+c_{0} \leq \ell_{\gamma} \leq s, v_{\gamma}^{ \pm} \in \mathscr{N}_{r} \Omega^{ \pm}\right\}$. By the above, since the integral of a function is equal to the integral on any Borel set containing its support, and since the integral of a nonnegative function is nondecreasing in the integration domain, there hence exists $c_{4}>0$ such that for all $T^{\prime} \geq T \geq 0$ and $\left.\left.\eta \in\right] 0,1\right]$, we have

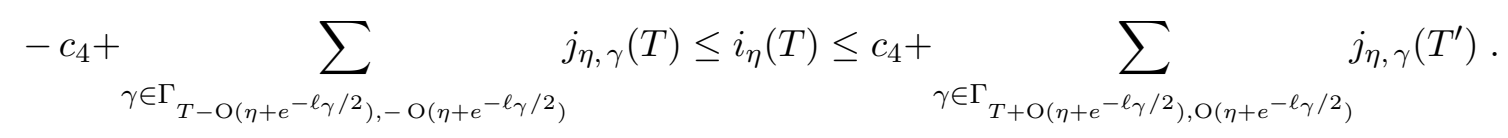

We will take $T^{\prime}$ to be of the form $T+\mathrm{O}\left(\eta+e^{-\ell_{\gamma} / 2}\right)$, for a bigger $\mathrm{O}(\cdot)$ than the one appearing in the index of the above summation.

Step 5: Conclusion. Let $\gamma \in \Gamma$ be such that $D^{-}$and $\gamma D^{+}$have a common perpendicular with length $\ell_{\gamma} \geq t_{0}+2+c_{0}$. Let us prove that for all $\epsilon>0$, if $\eta$ is small enough and $\ell_{\gamma}$ is large enough, then for every $T \geq \ell_{\gamma}+\mathrm{O}\left(\eta+e^{-\ell_{\gamma} / 2}\right.$ ) (with the enough's and $\mathrm{O}(\cdot)$ independent of $\gamma$ ), we have

$$
1-\epsilon \leq j_{\eta, \gamma}(T) \leq 1+\epsilon
$$

Note that $\widetilde{\sigma}^{ \pm}\left(\mathscr{N}_{\varepsilon}\left(\Omega^{\mp}\right)\right)$ and $\widetilde{\sigma}^{ \pm}\left(\mathscr{N}_{-\varepsilon}\left(\Omega^{\mp}\right)\right)$ tend to $\widetilde{\sigma}^{ \pm}\left(\Omega^{\mp}\right)$ as $\varepsilon \rightarrow 0$ (since $\widetilde{\sigma}^{ \pm}\left(\partial \Omega^{\mp}\right)=$ 0 as required in Step 1). Using Step 3 and Step 4, this will prove Equation (14), hence will complete the proof of Theorem 8 .

We say that $(\widetilde{M}, \Gamma)$ has radius-continuous strong stable/unstable ball masses if for every $\epsilon>0$, if $r \geq 1$ is close enough to 1 , then for every $v \in T^{1} \widetilde{M}$, if $B^{ \pm}(v, 1)$ meets the support of $\mu_{W^{ \pm}(v)}$, then

$$
\mu_{W^{ \pm}(v)}\left(B^{ \pm}(v, r)\right) \leq e^{\epsilon} \mu_{W^{ \pm}(v)}\left(B^{ \pm}(v, 1)\right) .
$$


We say that $(\widetilde{M}, \Gamma)$ has radius-Hölder-continuous strong stable/unstable ball masses if there exists $c \in] 0,1]$ and $c^{\prime}>0$ such that for every $\left.\left.\epsilon \in\right] 0,1\right]$, if if $B^{ \pm}(v, 1)$ meets the support of $\mu_{W^{ \pm}(v)}$, then

$$
\mu_{W^{ \pm}(v)}\left(B^{ \pm}(v, 1+\epsilon)\right) \leq e^{c^{\prime} \epsilon^{c}} \mu_{W^{ \pm}(v)}\left(B^{ \pm}(v, 1)\right) .
$$

When the sectional curvature has bounded derivatives and when $(\widetilde{M}, \Gamma)$ has radiusHölder-continuous strong stable/unstable ball masses, we will prove the following stronger statement: with a constant $c_{7}>0$ and functions $\mathrm{O}(\cdot)$ independent of $\gamma$, for all $\left.\left.\eta \in\right] 0,1\right]$ and $T \geq \ell_{\gamma}+\mathrm{O}\left(\eta+e^{-\ell_{\gamma} / 2}\right)$, we have

$$
j_{\eta, \gamma}(T)=\left(1+\mathrm{O}\left(\frac{e^{-\ell_{\gamma} / 2}}{2 \eta}\right)\right)^{2} e^{\mathrm{O}\left(\left(\eta+e^{-\ell_{\gamma} / 2}\right)^{c} 7\right)} .
$$

This stronger version will be needed for the error term estimate in Section 4 . In order to obtain Theorem 8, only the fact that $j_{\eta, \gamma}(T)$ tends to 1 as firstly $\ell_{\gamma}$ tends to $+\infty$, secondly $\eta$ tends to 0 is needed. A reader not interested in the error term may skip many technical details below.

Let $\eta \in] 0,1]$ and $T \geq \ell_{\gamma}+\mathrm{O}\left(\eta+e^{-\ell_{\gamma} / 2}\right)$. We start the proof of Equation 22 by defining parameters $s^{+}, s^{-}, s, v^{\prime}, v^{\prime \prime}$ associated with $(t, v) \in \mathscr{A}_{\eta, \gamma}(T)$.

$$
\begin{aligned}
& W^{\mathrm{ss}}\left(g^{t / 2} w^{-}\right) \underset{s^{-}}{W^{\mathrm{ss}}\left(v^{\prime}\right){ }^{W^{\mathrm{su}}\left(v^{\prime \prime}\right)}}{ }^{W^{\mathrm{su}}\left(g^{-t / 2} w^{+}\right)} \\
& \begin{array}{cccc} 
& s & v & g^{t / 2} w^{-} \\
& v^{\prime} & & \\
& & v^{\prime \prime} &
\end{array} \\
& x_{0} \quad v_{\gamma}^{0} \\
& W^{\mathrm{su}}\left(v_{\gamma}^{0}\right) \quad W^{\mathrm{ss}}\left(v_{\gamma}^{0}\right)
\end{aligned}
$$

We have $(t, v) \in \mathscr{A}_{\eta, \gamma}(T)$ if and only if there exist $\left.s^{ \pm} \in\right]-\eta, \eta[$ such that

$$
g^{\mp s^{\mp}} v \in B^{ \pm}\left(g^{ \pm t / 2} w^{\mp}, e^{-t / 2} R\right) .
$$

The notation $s^{ \pm}$coincides with the one in the proof of Lemma 7 (where $\left(D^{+}, w\right)$ has been replaced by $\left.\left(\gamma D^{+}, v\right)\right)$.

In order to define the parameters $s, v^{\prime}, v^{\prime \prime}$, we use the well known local product structure of the unit tangent bundle in negative curvature. If $v \in T^{1} M$ is close enough to $v_{\gamma}^{0}$ (in particular, $v_{-} \neq\left(v_{\gamma}^{0}\right)_{+}$and $\left.v_{+} \neq\left(v_{\gamma}^{0}\right)_{-}\right)$, then let $v^{\prime}=f_{H B_{-}\left(v_{\gamma}^{0}\right)}^{+}(v)$ be the unique element of $W^{-}\left(v_{\gamma}^{0}\right)$ such that $v_{+}^{\prime}=v_{+}$, let $v^{\prime \prime}=f_{H B_{+}\left(v_{\gamma}^{0}\right)}^{-}(v)$ be the unique element of $W^{+}\left(v_{\gamma}^{0}\right)$ such that $v_{-}^{\prime \prime}=v_{-}$, and let $s$ be the unique element of $\mathbb{R}$ such that $g^{-s} v \in W^{+}\left(v^{\prime}\right)$. The map $v \mapsto\left(s, v^{\prime}, v^{\prime \prime}\right)$ is a homeomorphism from a neighbourhood of $v_{\gamma}^{0}$ in $T^{1} \widetilde{M}$ to a neighbourhood of $\left(0, v_{\gamma}^{0}, v_{\gamma}^{0}\right)$ in $\mathbb{R} \times W^{-}\left(v_{\gamma}^{0}\right) \times W^{+}\left(v_{\gamma}^{0}\right)$. Note that if $v=g^{r} v_{\gamma}^{0}$ for some $r \in \mathbb{R}$ close to 0 , then

$$
w^{-}=v_{\gamma}^{-}, w^{+}=v_{\gamma}^{+}, s=r, v^{\prime}=v^{\prime \prime}=v_{\gamma}^{0}, s^{-}=\frac{\ell_{\gamma}-t}{2}+s, s^{+}=\frac{\ell_{\gamma}-t}{2}-s .
$$


Up to increasing $t_{0}$ (which does not change Step 4, up to increasing $c_{4}$ ), we may assume that for every $(t, v) \in \mathscr{A}_{\eta, \gamma}(T)$, the vector $v$ belongs to the domain of this local product structure of $T^{1} \widetilde{M}$ at $v_{\gamma}^{0}$.

The vectors $v, v^{\prime}, v^{\prime \prime}$ are close to $v_{\gamma}^{0}$ if $t$ is large and $\eta$ small, as the next result shows. We denote (also) by $d$ the Riemannian distance induced by Sasaki's metric on $T^{1} \widetilde{M}$.

Lemma 9 For every $(t, v) \in \mathscr{A}_{\eta, \gamma}(T)$, we have $d\left(v, v_{\gamma}^{0}\right), d\left(v^{\prime}, v_{\gamma}^{0}\right), d\left(v^{\prime \prime}, v_{\gamma}^{0}\right)=\mathrm{O}\left(\eta+e^{-t / 2}\right)$.

Proof. Consider the distance $d^{\prime}$ on $T^{1} \widetilde{M}$, defined by

$$
\forall v_{1}, v_{2} \in T^{1} \widetilde{M}, \quad d^{\prime}\left(v_{1}, v_{2}\right)=\max _{r \in[-1,0]} d\left(\pi\left(g^{r} v_{1}\right), \pi\left(g^{r} v_{2}\right)\right) .
$$

By (iii) in Step 4, we have $d\left(\pi(v), \pi\left(v_{\gamma}^{0}\right)\right)=\mathrm{O}\left(\eta+e^{-t / 2}\right)$. By Lemma 5, we have

$$
d\left(\pi\left(g^{-\frac{t}{2}-s^{-}} v\right), \pi\left(v_{\gamma}^{-}\right)\right) \leq d\left(\pi\left(g^{-\frac{t}{2}-s^{-}} v\right), \pi\left(w^{-}\right)\right)+d\left(\pi\left(w^{-}\right), \pi\left(v_{\gamma}^{-}\right)\right) \leq R+c_{0} e^{-t / 2} .
$$

By an exponential pinching argument, we hence have $d^{\prime}\left(v, v_{\gamma}^{0}\right)=\mathrm{O}\left(\eta+e^{-\ell_{\gamma} / 2}\right)$. Since $d$ and $d^{\prime}$ are equivalent (see [Bal, p. 70]), we therefore have $d\left(v, v_{\gamma}^{0}\right)=\mathrm{O}\left(\eta+e^{-\ell_{\gamma} / 2}\right)$.

For all $w \in T^{1} \widetilde{M}$ and $V \in T_{w} T^{1} \widetilde{M}$, we may uniquely write $V=V^{\text {su }}+V^{0}+V^{\text {ss }}$ with $V^{\mathrm{su}} \in T_{w} W^{-}(w), V^{0} \in \mathbb{R} \frac{d}{d t} \mid t_{0} g^{t} w$ and $V^{\mathrm{ss}} \in T_{w} W^{-}(w)$. By [PPS, §7.2], Sasaki's metric (with norm $\|\cdot\|$ ) is equivalent to the Riemannian metric with (product) norm

$$
\|V\|^{\prime}=\sqrt{\left\|V^{\mathrm{su}}\right\|^{2}+\left\|V^{0}\right\|^{2}+\left\|V^{\mathrm{ss}}\right\|^{2}} .
$$

By the dynamical local product structure of $T^{1} \widetilde{M}$ in the neighbourhood of $v_{\gamma}^{0}$ and by the definition of $v^{\prime}, v^{\prime \prime}$, the result follows, since the exponential map of $T^{1} \widetilde{M}$ at $v_{\gamma}^{0}$ is almost isometric close to 0 and the projection to a factor of a product norm is 1-Lipschitz.

We use the local product structure of the Bowen-Margulis measure to prove the following result.

Lemma 10 For every $(t, v) \in \mathscr{A}_{\eta, \gamma}(T)$, we have

$$
d t d \widetilde{m}_{\mathrm{BM}}(v)=e^{\mathrm{O}\left(\eta+e^{-\ell_{\gamma} / 2}\right)} d t d s d \mu_{W^{-}\left(v_{\gamma}^{0}\right)}\left(v^{\prime}\right) d \mu_{W^{+}\left(v_{\gamma}^{0}\right)}\left(v^{\prime \prime}\right) .
$$

Proof. Since the above parameter $s$ differs, when $v_{-}, v_{+}$are fixed, only by a constant from the time parameter in Hopf's parametrisation, we have

$$
d \widetilde{m}_{\mathrm{BM}}(v)=\frac{e^{-\delta\left(\beta_{v_{-}}\left(\pi(v), x_{0}\right)+\beta_{v_{+}}\left(\pi(v), x_{0}\right)\right)}}{e^{-\delta\left(\beta_{v_{+}^{\prime}}\left(\pi\left(v^{\prime}\right), x_{0}\right)+\beta_{v_{-}^{\prime \prime}}\left(\pi\left(v^{\prime \prime}\right), x_{0}\right)\right)}} d \mu_{W^{-}\left(v_{\gamma}^{0}\right)}\left(v^{\prime}\right) d \mu_{W^{+}\left(v_{\gamma}^{0}\right)}\left(v^{\prime \prime}\right) d t .
$$

As $\pi: T^{1} \widetilde{M} \rightarrow \widetilde{M}$ is 1-Lipschitz, and since $v_{+}=v_{+}^{\prime}$ and $v_{-}=v_{-}^{\prime \prime}$, the claim follows from Lemma 9 and the fact that the map $x \mapsto \beta_{\xi}\left(x, x_{0}\right)$ is 1-Lipschitz for every $\xi \in \partial_{\infty} \widetilde{M}$.

When $\ell_{\gamma}$ is large, the submanifold $g^{\ell_{\gamma} / 2} \Omega^{-}$has a second order contact at $v_{\gamma}^{0}$ with $W^{-}\left(v_{\gamma}^{0}\right)$ and similarly, $g^{-\ell_{\gamma} / 2} \Omega^{+}$has a second order contact at $v_{\gamma}^{0}$ with $W^{+}\left(v_{\gamma}^{0}\right)$. Let $P_{\gamma}$ be the plane domain of $(t, s) \in \mathbb{R}^{2}$ such that there exist $\left.s^{ \pm} \in\right]-\eta, \eta\left[\right.$ with $s^{\mp}=$ 
$\frac{\ell_{\gamma}-t}{2} \pm s+\mathrm{O}\left(e^{-\ell_{\gamma} / 2}\right)$. Note that its area is $\left(2 \eta+\mathrm{O}\left(e^{-\ell_{\gamma} / 2}\right)\right)^{2}$. By the above, we have (with the obvious meaning of a double inclusion)

$$
\mathscr{A}_{\eta, \gamma}(T)=P_{\gamma} \times B^{-}\left(v_{\gamma}^{0}, r_{\ell_{\gamma}} e^{\mathrm{O}\left(\eta+e^{-\ell_{\gamma} / 2}\right)}\right) \times B^{+}\left(v_{\gamma}^{0}, r_{\ell_{\gamma}} e^{\mathrm{O}\left(\eta+e^{-\ell_{\gamma} / 2}\right)}\right) .
$$

By Lemma 10, we hence have

$$
\begin{aligned}
& \int_{\mathscr{A}_{\eta, \gamma}(T)} d t d \widetilde{m}_{\mathrm{BM}}(v)=e^{\mathrm{O}\left(\eta+e^{-\ell_{\gamma} / 2}\right)}\left(2 \eta+\mathrm{O}\left(e^{-\ell_{\gamma} / 2}\right)\right)^{2} \times \\
& \mu_{W^{-}\left(v_{\gamma}^{0}\right)}\left(B^{-}\left(v_{\gamma}^{0}, r_{\ell_{\gamma}} e^{\mathrm{O}\left(\eta+e^{-\ell_{\gamma} / 2}\right)}\right)\right) \mu_{W^{+}\left(v_{\gamma}^{0}\right)}\left(B^{+}\left(v_{\gamma}^{0}, r_{\ell_{\gamma}} e^{\mathrm{O}\left(\eta+e^{-\ell_{\gamma} / 2}\right)}\right)\right) .
\end{aligned}
$$

The last ingredient of the proof of Step 5 is the following continuity property of strong stable and strong unstable ball volumes as their center varies (see [Rob, Lem. 1.16], [PPS, Prop. 10.16] for related properties, though we need a more precise control for the error term in Section 4 .

Lemma 11 Assume that $(\widetilde{M}, \Gamma)$ has radius-continuous strong stable/unstable ball masses. There exists $c_{5}>0$ such that for every $\epsilon>0$, if $\eta$ is small enough and $\ell_{\gamma}$ large enough, then for every $(t, v) \in \mathscr{A}_{\eta, \gamma}(T)$, we have

$$
\mu_{W^{ \pm}\left(w_{t}^{\mp}\right)}\left(B^{ \pm}\left(w_{t}^{\mp}, r_{t}\right)\right)=e^{\mathrm{O}\left(\epsilon^{c_{5}}\right)} \mu_{W^{ \pm}\left(v_{\gamma}^{0}\right)}\left(B^{ \pm}\left(v_{\gamma}^{0}, r_{\ell_{\gamma}}\right)\right) .
$$

If we furthermore assume that the sectional curvature of $\widetilde{M}$ has bounded derivatives and that $(\widetilde{M}, \Gamma)$ has radius-Hölder-continuous strong stable/unstable ball masses, then we may replace $\epsilon$ by $\left(\eta+e^{-\ell_{\gamma} / 2}\right)^{c_{6}}$ for some constant $c_{6}>0$.

Proof. We prove the claim for $\mathscr{W}^{+}$, the one for $\mathscr{W}^{-}$follows similarly. The final statement is only used for the error estimates in Section 4.

$$
\begin{array}{ccccc} 
& & & & B^{+}\left(w^{-}, R\right) \\
\mathrm{O}\left(e^{-\ell_{\gamma} / 2}\right) & w^{-} & t / 2 & w_{t}^{-} & \\
& & & \mathrm{O}\left(\eta+e^{-\ell_{\gamma} / 2}\right) & w^{-} \\
& v_{\gamma}^{-} & \ell_{\gamma} / 2 & v_{\gamma}^{0} & B^{+}\left(v_{\gamma}^{-}, R e^{\mathrm{O}\left(\eta+e^{\ell_{\gamma} / 2}\right)}\right)
\end{array}
$$

Using Equation (5) and the scaling properties of skinning measure, we have

$$
\mu_{W^{+}\left(w_{t}^{-}\right)}\left(B^{+}\left(w_{t}^{-}, r_{t}\right)\right)=e^{-\delta t / 2} \mu_{W^{+}\left(w^{-}\right)}\left(B^{+}\left(w^{-}, R\right)\right)
$$

and similarly, for every $a>0$,

$$
\mu_{W^{+}\left(v_{\gamma}^{0}\right)}\left(B^{+}\left(v_{\gamma}^{0}, a r_{t}\right)\right)=e^{-\delta t / 2} \mu_{W^{+}\left(v_{\gamma}^{-}\right)}\left(B^{+}\left(v_{\gamma}^{-}, a R\right)\right) .
$$

Let $h^{-}: B^{+}\left(w^{-}, R\right) \rightarrow W^{+}\left(v_{\gamma}^{-}\right)$be the map such that $\left(h^{-}(v)\right)_{-}=v_{-}$, which is well defined and a homeomorphism onto its image if $\ell_{\gamma}$ is large enough (since $R$ is fixed). By [PP5, Prop. 5] where $C=H B_{+}\left(w^{-}\right), C^{\prime}=H B_{+}\left(v_{\gamma}^{-}\right)$, we have, for every $v \in B^{+}\left(w^{-}, R\right)$,

$$
d \mu_{W^{+}\left(w^{-}\right)}(v)=e^{-\delta \beta_{v_{-}}\left(\pi(v), \pi\left(h^{-}(v)\right)\right)} d \mu_{W^{+}\left(v_{\gamma}^{-}\right)}\left(h^{-}(v)\right) .
$$


Let us fix $\epsilon>0$. The strong stable balls of radius $R$ centered at $w^{-}$and $v_{\gamma}^{-}$are very close (see the above picture). More precisely, recall that $R$ is fixed, and that, as seen above, $d\left(\pi\left(w^{-}\right), \pi\left(v_{\gamma}^{-}\right)\right)=\mathrm{O}\left(e^{-\ell_{\gamma} / 2}\right)$ and $d\left(\pi\left(g^{t / 2} w^{-}\right), \pi\left(g^{\ell_{\gamma} / 2} v_{\gamma}^{-}\right)\right)=\mathrm{O}\left(\eta+e^{-\ell_{\gamma} / 2}\right)$. Therefore we have $d\left(\pi(v), \pi\left(h^{-}(v)\right)\right) \leq \epsilon$ for every $v \in B^{+}\left(w^{-}, R\right)$ if $\eta$ is small enough and $\ell_{\gamma}$ large enough. If furthermore the sectional curvature has bounded derivatives, then by Anosov's arguments (see for instance [PPS, Theo. 7.3]) the strong stable foliation is Hölder-continuous. Hence we have $d\left(\pi(v), \pi\left(h^{-}(v)\right)\right)=\mathrm{O}\left(\left(\eta+e^{-\ell_{\gamma} / 2}\right)^{c_{5}}\right)$ for every $v \in$ $B^{+}\left(w^{-}, R\right)$, for some constant $c_{5}>0$, under the additional hypothesis on the curvature. We also have $h^{-}\left(B^{+}\left(w^{-}, R\right)\right)=B^{+}\left(v_{\gamma}^{-}, R e^{\mathrm{O}(\epsilon)}\right)$ and, under the additional hypothesis on the curvature, $h^{-}\left(B^{+}\left(w^{-}, R\right)\right)=B^{+}\left(v_{\gamma}^{-}, R e^{\mathrm{O}\left(\left(\eta+e^{-\ell_{\gamma} / 2}\right)^{c_{5}}\right)}\right)$. Assume in what follows that $\epsilon=\left(\eta+e^{-\ell_{\gamma} / 2}\right)^{c_{5}}$ under the additional hypothesis on the curvature. Since $\left|\beta_{\xi}(x, y)\right| \leq$ $d(x, y)$ for all $x, y \in \widetilde{M}$ and $\xi \in \partial_{\infty} \widetilde{M}$, we hence have, for every $v \in B^{+}\left(w^{-}, R\right)$,

$$
d \mu_{W^{+}\left(w^{-}\right)}(v)=e^{\mathrm{O}(\epsilon)} d \mu_{W^{+}\left(v_{\gamma}^{-}\right)}\left(h^{-}(v)\right) .
$$

The result follows by Equations (25), (26) and the continuity property in the radius.

Now Lemma 11 (with $\epsilon$ as in its statement, and when its hypotheses are satisfied) implies that

$$
\begin{aligned}
& \iint_{(t, v) \in \mathscr{A}_{\eta, \gamma}(T)} \frac{d t d \widetilde{m}_{\mathrm{BM}}(v)}{\mu_{W^{+}\left(w_{t}^{-}\right)}\left(B^{+}\left(w_{t}^{-}, r_{t}\right)\right) \mu_{W^{-}\left(w_{t}^{+}\right)}\left(B^{-}\left(w_{t}^{+}, r_{t}\right)\right)} \\
= & \frac{e^{\mathrm{O}\left(\epsilon^{c 5}\right)} \iint_{(t, v) \in \mathscr{A}_{\eta, \gamma}(T)} d t d \widetilde{m}_{\mathrm{BM}}(v)}{\mu_{W^{+}\left(v_{\gamma}^{0}\right)}\left(B^{+}\left(v_{\gamma}^{0}, r_{t}\right)\right) \mu_{W^{-}\left(v_{\gamma}^{0}\right)}\left(B^{-}\left(v_{\gamma}^{0}, r_{t}\right)\right)} .
\end{aligned}
$$

By Equation (21) and Equation (24), we hence have

$$
j_{\eta, \gamma}(T)=e^{\mathrm{O}\left(\eta+e^{-\ell_{\gamma} / 2}\right)} e^{\mathrm{O}\left(\epsilon^{c_{5}}\right)} \frac{\left(2 \eta+\mathrm{O}\left(e^{-\ell_{\gamma} / 2}\right)\right)^{2}}{(2 \eta)^{2}}
$$

under the technical assumptions of Lemma 11. The assumption on radius-continuity of strong stable/unstable ball masses can be bypassed using bump functions, as explained in [Rob, p. 81], which concludes the proof of Step 5.

\subsection{Equidistribution of endvectors of common perpendiculars in $T^{1} M$}

We now deduce from Theorem 8 , which is an equidistribution result in $T^{1} \widetilde{M} \times T^{1} \widetilde{M}$, an equidistribution result in its quotient $T^{1} M \times T^{1} M$ by the action of $\Gamma \times \Gamma$.

Let $\mathscr{D}=\left(D_{i}\right)_{i \in I}$ be a locally finite $\Gamma$-equivariant family of nonempty proper closed convex subsets of $\widetilde{M}$. Let $\Omega=\left(\Omega_{i}\right)_{i \in I}$ be a $\Gamma$-equivariant family of subsets of $T^{1} \widetilde{M}$, where $\Omega_{i}$ is a measurable subset of $\partial_{ \pm}^{1} D_{i}$ for all $i \in I$ (the sign \pm being constant). Then

$$
\tilde{\sigma}_{\Omega}^{ \pm}=\left.\sum_{i \in I / \sim} \tilde{\sigma}_{D_{i}}^{ \pm}\right|_{\Omega_{i}}
$$

is a well-defined (independent of the choice of representatives in $I / \sim$ ), $\Gamma$-invariant, locally finite measure on $T^{1} \widetilde{M}$ whose support is contained in $\bigcup_{i \in I / \sim} \Omega_{i}$. The measure $\widetilde{\sigma}_{\Omega}^{ \pm}$induces a locally finite measure on $T^{1} M$, denoted by $\sigma_{\Omega}^{ \pm}$. The measures induced by $\tilde{\sigma}_{\mathscr{D}}^{ \pm}$on $T^{1} M=$ 
$\Gamma \backslash T^{1} \widetilde{M}$ are called the inner/outer skinning measures of $\mathscr{D}$ on $T^{1} M$. If $\widetilde{x}_{0}$ has stabiliser $\Gamma_{\widetilde{x}_{0}}$ and maps to $x_{0} \in M$, if $\mathscr{D}=\left(\gamma \widetilde{x}_{0}\right)_{\gamma \in \Gamma}$, if $M$ has dimension $n$, constant curvature and finite volume, then, normalising the Patterson-Sullivan density so that $\left\|\mu_{x}\right\|=\operatorname{Vol}\left(\mathbb{S}^{n-1}\right)$, we have $\sigma_{\mathscr{D}}^{ \pm}=\operatorname{Vol}_{T_{x_{0}}^{1} M}$ and $\left\|\sigma_{\mathscr{D}}^{ \pm}\right\|=\frac{\operatorname{Vol}\left(\mathbb{S}^{n-1}\right)}{\operatorname{Card}\left(\Gamma_{\widetilde{x}_{0}}\right)}$. See Section 6 for other examples.

Given $v \in T^{1} M$, we define the natural multiplicity of $v$ with respect to $\Omega$ by

$$
m_{\Omega}(v)=\frac{\operatorname{Card}\left\{i \in I / \sim: \widetilde{v} \in \Omega_{i}\right\}}{\operatorname{Card}\left(\operatorname{Stab}_{\Gamma} \widetilde{v}\right)},
$$

for any preimage $\widetilde{v}$ of $v$ in $T^{1} \widetilde{M}$. The numerator and the denominator are finite, by the local finiteness of $\mathscr{D}$ and the discreteness of $\Gamma$, and they depend only on the orbit $\Gamma \widetilde{v}$. The numerator takes into account the multiplicities of the images of the elements of $\mathscr{D}$ in $T^{1} M$. Note that if $\Gamma$ is torsion-free, if $\Omega=\partial_{ \pm}^{1} \mathscr{D}$, if for every $i \in I$ the quotient $\Gamma_{D_{i}} \backslash D_{i}$ of $D_{i}$ by its stabiliser $\Gamma_{D_{i}}$ maps injectively in $M=\Gamma \backslash \widetilde{M}$ (by the map induced by the inclusion of $D_{i}$ in $\left.M\right)$, and if for every $i, j \in I$ such that $j \notin \Gamma i$, the intersection $D_{i} \cap D_{j}$ is empty, then the nonzero multiplicities $m_{\Omega}(v)$ are all equal to 1 .

Given $t>0$ and two unit tangent vectors $v, w \in T^{1} M$, let

$$
n_{t}(v, w)=\sum_{\alpha} \operatorname{Card}\left(\Gamma_{\alpha}\right)
$$

where the sum ranges over the locally geodesic paths $\alpha:[0, s] \rightarrow M$ such that $\dot{\alpha}(0)=v$, $\dot{\alpha}(s)=w$ and $s \in] 0, t]$, and $\Gamma_{\alpha}$ is the stabiliser in $\Gamma$ of any geodesic path $\widetilde{\alpha}$ in $\widetilde{M}$ mapping to $\alpha$ by the quotient map $\widetilde{M} \rightarrow M$. If $\Gamma$ is torsion free, then $n_{t}(v, w)$ is precisely the number of locally geodesic paths having $v$ and $w$ as initial and terminal tangent vectors respectively, with length at most $t$.

Let $\Omega^{ \pm}=\left(\Omega_{i}^{ \pm}\right)_{i \in I^{ \pm}}$be $\Gamma$-equivariant families of subsets of $T^{1} \widetilde{M}$, where $\Omega_{k}^{\mp}$ is a measurable subset of $\partial_{ \pm}^{1} D_{k}^{\mp}$ for all $k \in I^{\mp}$. We will denote by $\mathscr{N}_{\Omega^{-}, \Omega^{+}}(t)$ the number of common perpendiculars whose initial vectors belong to the images in $M$ of the elements of $\Omega^{-}$and terminal vectors to the images in $M$ of the elements of $\Omega^{+}$, counted with multiplicities:

$$
\mathscr{N}_{\Omega^{-}, \Omega^{+}}(t)=\sum_{v, w \in T^{1} M} m_{\Omega^{-}}(v) m_{\Omega^{+}}(w) n_{t}(v, w) .
$$

When $\Omega^{ \pm}=\partial_{\mp}^{1} \mathscr{D}^{ \pm}$, we denote $\mathscr{N}_{\Omega^{-}, \Omega^{+}}$by $\mathscr{N}_{\mathscr{D}^{-}, \mathscr{D}^{+}}$. If $\Gamma$ has no torsion, if $\mathscr{D}^{ \pm}=\left(\gamma \widetilde{D}^{ \pm}\right)_{\gamma \in \Gamma}$ where $\widetilde{D}^{ \pm}$is a nonempty proper closed convex subset of $\widetilde{M}$ (such that the family $\mathscr{D}^{ \pm}$is locally finite), and if $D^{ \pm}$is the image of $\widetilde{D}^{ \pm}$by the covering map $\widetilde{M} \rightarrow M=\Gamma \backslash \widetilde{M}$ (which is a nonempty proper properly immersed closed locally convex subset of $M$ ), then $\mathscr{N}_{\mathscr{D}^{-}, \mathscr{D}^{+}}$ is the counting function $\mathscr{N}_{D^{-}, D^{+}}$given in the introduction.

Recall that the narrow topology (also called weak topology) on the set $\mathscr{M}_{\mathrm{f}}(Y)$ of finite measures on a Polish space $Y$ is the smallest topology such that, for every bounded continuous map $g: Y \rightarrow \mathbb{R}$, the map from $\mathscr{M}_{\mathrm{f}}(Y)$ to $\mathbb{R}$ defined by $\mu \mapsto \mu(g)$ is continuous.

Corollary 12 Let $\widetilde{M}, \Gamma, \mathscr{D}^{-}, \mathscr{D}^{+}$be as in Theorem 8 . Then,

$$
\lim _{t \rightarrow+\infty} \delta\left\|m_{\mathrm{BM}}\right\| e^{-\delta t} \sum_{v, w \in T^{1} M} m_{\partial_{+}^{1} \mathscr{D}^{-}}(v) m_{\partial_{-}^{1} \mathscr{D}^{+}}(w) n_{t}(v, w) \Delta_{v} \otimes \Delta_{w}=\sigma_{\mathscr{D}^{-}}^{+} \otimes \sigma_{\mathscr{D}^{+}}^{-}
$$


for the weak-star convergence of measures on the locally compact space $T^{1} M \times T^{1} M$. If $\sigma_{\mathscr{D}^{-}}^{+}$and $\sigma_{\mathscr{D}^{+}}^{-}$are finite, the result also holds for the narrow convergence.

Furthermore, for all $\Gamma$-equivariant families $\Omega^{ \pm}=\left(\Omega_{k}^{ \pm}\right)_{k \in I^{ \pm}}$of subsets of $T^{1} \widetilde{M}$ with $\Omega_{k}^{\mp}$ a Borel subset of $\partial_{ \pm}^{1} D_{k}^{\mp}$ for all $k \in I^{\mp}$, with nonzero finite skinning measure and with boundary in $\partial_{ \pm}^{1} D_{k}^{\mp}$ of zero skinning measure, we have as $t \rightarrow+\infty$

$$
\mathscr{N}_{\Omega^{-}, \Omega^{+}}(t) \sim \frac{\left\|\sigma_{\Omega^{-}}^{+}\right\|\left\|\sigma_{\Omega^{+}}^{-}\right\|}{\delta\left\|m_{\mathrm{BM}}\right\|} e^{\delta t}
$$

Proof. Note that the sum in Equation (27) is locally finite, hence it defines a locally finite measure on $T^{1} M \times T^{1} M$. We are going to rewrite the sum in the statement of Theorem 8 in a way which makes it easier to push it down from $T^{1} \widetilde{M} \times T^{1} \widetilde{M}$ to $T^{1} M \times T^{1} M$.

For every $\widetilde{v} \in T^{1} \widetilde{M}$, let

$$
m^{\mp}(\widetilde{v})=\operatorname{Card}\left\{k \in I^{\mp} / \sim: \widetilde{v} \in \partial_{ \pm}^{1} D_{k}^{\mp}\right\},
$$

so that for every $v \in T^{1} M$, the multiplicity of $v$ with respect to the family $\partial_{ \pm}^{1} \mathscr{D}^{\mp}$ is

$$
m_{\partial_{ \pm}^{1} \mathscr{D ^ { \mp }}}(v)=\frac{m^{\mp}(\widetilde{v})}{\operatorname{Card}\left(\operatorname{Stab}_{\Gamma} \widetilde{v}\right)}
$$

for any preimage $\widetilde{v}$ of $v$ in $T^{1} \widetilde{M}$.

For all $\gamma \in \Gamma$ and $\widetilde{v}, \widetilde{w} \in T^{1} \widetilde{M}$, there exists $(i, j) \in\left(I^{-} / \sim\right) \times\left(I^{+} / \sim\right)$ such that $\widetilde{v}=v_{i, \gamma j}^{-}$ and $\widetilde{w}=v_{\gamma^{-1} i, j}^{+}=\gamma^{-1} v_{i, \gamma j}^{+}$if and only if $\gamma \widetilde{w} \in g^{\mathbb{R}} \widetilde{v}$, there exists $i^{\prime} \in I^{-} / \sim$ such that $\widetilde{v} \in \partial_{+}^{1} D_{i^{\prime}}^{-}$and there exists $j^{\prime} \in I^{+} / \sim$ such that $\gamma \widetilde{w} \in \partial_{-}^{1} D_{j^{\prime}}^{+}$. Then the choice of such elements $(i, j)$, as well as $i^{\prime}$ and $j^{\prime}$, is free. We hence have

$$
\begin{aligned}
& \sum_{\substack{i \in I^{-} / \sim, j \in I^{+} / \widetilde{\sim}, \gamma \in \Gamma \\
0<\ell\left(\alpha_{i, \gamma j}\right) \leq t, v_{i, \gamma j}^{-}=\widetilde{v}, v_{\gamma}^{+} 1_{i, j}}} \Delta_{v_{i, \gamma j}^{-}} \otimes \Delta_{v_{\gamma^{-1}+, j}^{+}} \\
= & \sum_{\substack{\gamma \in \Gamma, 0<s \leq t \\
\gamma \widetilde{w}=g^{s} \widetilde{v}}} \operatorname{Card}\left\{(i, j) \in\left(I^{-} / \sim\right) \times\left(I^{+} / \sim\right): v_{i, \gamma j}^{-}=\widetilde{v}, v_{\gamma^{-1} i, j}^{+}=\widetilde{w}\right\} \Delta_{\widetilde{v}} \otimes \Delta_{\widetilde{w}} \\
= & \sum_{\substack{\gamma \in \Gamma, 0<s \leq t \\
\gamma \widetilde{w}=g^{s} \widetilde{v}}} m^{-}(\widetilde{v}) m^{+}(\gamma \widetilde{w}) \Delta_{\widetilde{v}} \otimes \Delta_{\widetilde{w}} .
\end{aligned}
$$

Therefore

$$
\begin{aligned}
& \sum_{\substack{i \in I^{-} / \sim, j \in I^{+} / \sim, \gamma \in \Gamma \\
0<\ell\left(\alpha_{i, \gamma j}\right) \leq t}} \Delta_{v_{i, \gamma j}^{-}} \otimes \Delta_{v_{\gamma^{-1} i, j}^{+}} \\
= & \left.\left.\sum_{\widetilde{v}, \widetilde{w} \in T^{1} \widetilde{M}} \operatorname{Card}\{\gamma \in \Gamma: \exists s \in] 0, t\right], \gamma \widetilde{w}=g^{s} \widetilde{v}\right\} m^{-}(\widetilde{v}) m^{+}(\widetilde{w}) \Delta_{\widetilde{v}} \otimes \Delta_{\widetilde{w}} .
\end{aligned}
$$

By definition, $\sigma_{\mathscr{D} \mp}^{ \pm}$is the measure on $T^{1} M$ induced by the $\Gamma$-invariant measure $\widetilde{\sigma}_{\mathscr{D} \mp}^{ \pm}$, see [PPS, p. 28]. Thus the claim on weak-star convergence follows from Theorem 8 and Equation (14) (after a similar reduction as in Step 1 of the proof of Theorem 8). Since no compactness assumptions were made on $\Omega^{ \pm}$in this Step 1 in order to get Equation (14), the narrow convergence claim in Corollary 12 follows. 
Using the continuity of the pushforwards of measures for the weak-star and the narrow topologies, applied to the basepoint maps $\pi \times \pi$ from $T^{1} \widetilde{M} \times T^{1} \widetilde{M}$ to $\widetilde{M} \times \widetilde{M}$, and from $T^{1} M \times T^{1} M$ to $M \times M$, we have the following result of equidistribution of the ordered pairs of endpoints of common perpendiculars between two equivariant families of convex sets in $\widetilde{M}$ or two families of locally convex sets in $M$. When $M$ has constant curvature and finite volume, $\mathscr{D}^{-}$is the $\Gamma$-orbit of a point and $\mathscr{D}^{+}$is the $\Gamma$-orbit of a totally geodesic cocompact submanifold, this result is due to Herrmann [Her].

Corollary 13 Let $\widetilde{M}, \Gamma, \mathscr{D}^{-}, \mathscr{D}^{+}$be as in Theorem 8 . Then

$$
\lim _{t \rightarrow+\infty} \delta\left\|m_{\mathrm{BM}}\right\| e^{-\delta t} \sum_{\substack{i \in I^{-} / \mathcal{N}, j \in I^{+} / \tilde{\sim}, \gamma \in \Gamma \\ 0<\ell\left(\alpha_{i, \gamma j} \leq t\right.}} \Delta_{\pi\left(v_{i, \gamma j}^{-}\right)} \otimes \Delta_{\pi\left(v_{\gamma^{-1}, j}^{+}\right)}=\pi_{*} \widetilde{\sigma}_{\mathscr{D}^{-}}^{+} \otimes \pi_{*} \widetilde{\sigma}_{\mathscr{D}^{+}}^{-},
$$

for the weak-star convergence of measures on the locally compact space $\widetilde{M} \times \widetilde{M}$, and

$$
\begin{aligned}
& \lim _{t \rightarrow+\infty} \delta\left\|m_{\mathrm{BM}}\right\| e^{-\delta t} \sum_{v, w \in T^{1} M} m_{\partial_{+}^{1} \mathscr{D}^{-}}(v) m_{\partial_{-}^{1} \mathscr{D}^{+}}(w) n_{t}(v, w) \Delta_{\pi(v)} \otimes \Delta_{\pi(w)} \\
& =\pi_{*} \sigma_{\mathscr{D}^{-}}^{+} \otimes \pi_{*} \sigma_{\mathscr{D}^{+}}^{-}
\end{aligned}
$$

for the weak-star convergence of measures on $M \times M$. If the measures $\sigma_{\mathscr{D} \text { F }}^{ \pm}$are finite, then the above claim holds for the narrow convergence of measures on $M \times M$.

Before proving Theorems 1 and 2 in the introduction, we recall the definition of a proper nonempty properly immersed closed locally convex subset $D^{ \pm}$in a negatively curved complete connected Riemannian manifold $N$ : it is a locally convex (not necessarily connected) geodesic metric space $D^{ \pm}$endowed with a continuous map $f^{ \pm}: D^{ \pm} \rightarrow N$ such that, if $\widetilde{N} \rightarrow N$ and $\widetilde{D}^{ \pm} \rightarrow D^{ \pm}$are (locally isometric) universal covers, if $\widetilde{f}^{ \pm}: \widetilde{D}^{ \pm} \rightarrow \widetilde{N}$ is a lift of $f^{ \pm}$, then $\widetilde{f}^{ \pm}$is, on each connected component of $\widetilde{D}^{ \pm}$, an isometric embedding whose image is a proper nonempty closed locally convex subset of $\widetilde{N}$, and the family of images, under the covering group of $\widetilde{N} \rightarrow N$, of the images by $\widetilde{f}^{ \pm}$of the connected components of $\widetilde{D}^{ \pm}$is locally finite.

Proof of Theorems 1 and 2. Let $I^{ \pm}=\Gamma \times \pi_{0}\left(\widetilde{D}^{ \pm}\right)$with the action of $\Gamma$ defined by $\gamma \cdot(\alpha, c)=(\gamma \alpha, c)$ for all $\gamma, \alpha \in \Gamma$ and every component $c$ of $\widetilde{D}^{ \pm}$. Consider the families $\mathscr{D}^{ \pm}=\left(D_{k}^{ \pm}\right)_{k \in I^{ \pm}}$where $D_{k}^{ \pm}=\alpha \widetilde{f}^{ \pm}(c)$ if $k=(\alpha, c)$. Then $\mathscr{D}^{ \pm}$are $\Gamma$-equivariant families of nonempty proper closed convex subsets of $\widetilde{M}$, which are locally finite since $D^{ \pm}$are properly immersed in $M$. Theorems 1 and 2 then follow from Corollary 12 .

Corollary 14 Let $\widetilde{M}, \Gamma, \mathscr{D}^{-}, \mathscr{D}^{+}$be as in Theorem 8 . Assume that $\sigma_{\mathscr{D} \mp}^{ \pm}$are finite and nonzero. Then

$$
\lim _{s \rightarrow+\infty} \lim _{t \rightarrow+\infty} \frac{\delta\left\|m_{\mathrm{BM}}\right\|^{2} e^{-\delta t}}{\left\|\sigma_{\mathscr{D}^{-}}^{+}\right\|\left\|\sigma_{\mathscr{D}^{+}}^{-}\right\|} \sum_{v \in T^{1} M} m_{\partial_{+}^{1} \mathscr{D}^{-}}(v) n_{t, \mathscr{D}^{+}}(v) \Delta_{g^{s} v}=m_{\mathrm{BM}},
$$

where

$$
n_{t, \mathscr{D}}+(v)=\sum_{w \in T^{1} M} m_{\partial_{-}^{1} \mathscr{D}^{+}}(w) n_{t}(v, w)
$$

is the number (counted with multiplicities) of locally geodesic paths in $M$ of length at most $t$, with initial vector $v$, arriving perpendicularly to $\mathscr{D}^{+}$. 
Proof. For every $s \in \mathbb{R}$, by Corollary 12 , using the continuity of the pushforwards of measures by the first projection $(v, w) \mapsto v$ from $T^{1} M \times T^{1} M$ to $T^{1} M$, and by the geodesic flow on $T^{1} M$ at time $s$, since $\left(g^{s}\right)_{*} \Delta_{v}=\Delta_{g^{s} v}$, we have

$$
\lim _{t \rightarrow+\infty} \delta\left\|m_{\mathrm{BM}}\right\| e^{-\delta t} \sum_{v \in T^{1} M} m_{\partial_{+}^{1} \mathscr{D}^{-}}(v) n_{t, \mathscr{D}^{+}}(v) \Delta_{g^{s} v}=\left(g^{s}\right)_{*} \sigma_{\mathscr{D}^{-}}^{+}\left\|\sigma_{\mathscr{D}^{+}}^{-}\right\| .
$$

The result then follows from [PP5, Thm.1].

\section{Error terms}

Let $\widetilde{M}, x_{0}, \Gamma, \delta$ and $M$ be as in the beginning of Section 2. We assume that the BowenMargulis measure $m_{\mathrm{BM}}$ is finite, and we define $\overline{m_{\mathrm{BM}}}=\frac{m_{\mathrm{BM}}}{\left\|m_{\mathrm{BM}}\right\|}$.

In this section, we give bounds for the error term in the equidistribution and counting results of the previous section when the geodesic flow is exponentially mixing and the (strong) stable and unstable foliations are assumed to be at least Hölder-continuous.

There are two types of exponential mixing results available in this context. Firstly, when $\widetilde{M}$ is a symmetric space, then the boundary at infinity of $\widetilde{M}$, the strong unstable, unstable, stable, and strong stable foliations of $T^{1} \widetilde{M}$ are smooth. Hence talking about leafwise $\mathscr{C}^{\ell}$ functions on $T^{1} M$ makes sense. Let $\mathscr{C}_{c}^{\ell}\left(T^{1} M\right)$ be the space of $\mathscr{C}^{\ell}$ functions on $T^{1} M$ with compact support and by $\|\psi\|_{\ell}$ the Sobolev $W^{\ell, 2}$-norm of $\psi \in \mathscr{C}_{c}^{\ell}\left(T^{1} M\right)$.

For $\ell \in \mathbb{N}$, we say that the geodesic flow on $T^{1} M$ is exponentially mixing for the Sobolev regularity $\ell$ if there exist $c, \kappa>0$ such that for all $\phi, \psi \in \mathscr{C}_{c}^{\ell}\left(T^{1} M\right)$ and $t \in \mathbb{R}$, we have

$$
\left|\int_{T^{1} M} \phi \circ g^{-t} \psi d \overline{m_{\mathrm{BM}}}-\int_{T^{1} M} \phi d \overline{m_{\mathrm{BM}}} \int_{T^{1} M} \psi d \overline{m_{\mathrm{BM}}}\right| \leq c e^{-\kappa|t|}\|\psi\|_{\ell}\|\phi\|_{\ell} .
$$

When $\Gamma$ is an arithmetic lattice (the Bowen-Margulis measure then coincides, up to a multiplicative constant, with the Liouville measure), this property, for some $\ell \in \mathbb{N}$, follows from [KM1, Theorem 2.4.5], with the help of [Clo, Theorem 3.1] to check its spectral gap property, and of [KM2, Lemma 3.1] to deal with finite cover problems. When $M$ has finite volume, the conditional measures on the strong stable/unstable leaves are homogeneous, hence $(\widetilde{M}, \Gamma)$ has radius-Hölder-continuous strong stable/unstable ball masses.

Secondly, when $\widetilde{M}$ has pinched negative sectional curvature with bounded derivatives, then the boundary at infinity of $\widetilde{M}$, the strong unstable, unstable, stable, and strong stable foliations of $T^{1} \widetilde{M}$ are only Hölder-smooth (see for instance [Bri] when $\widetilde{M}$ has a compact quotient and [PPS, Theo. 7.3]). Hence it is appropriate to consider Hölder functions on $T^{1} \widetilde{M}$. For every $\left.\alpha \in\right] 0,1\left[\right.$, let $\mathscr{C}_{\mathrm{c}}^{\alpha}(X)$ be the space of $\alpha$-Hölder-continuous real-valued functions with compact support on a metric space $(X, d)$, endowed with the Hölder norm

$$
\|f\|_{\alpha}=\|f\|_{\infty}+\sup _{x, y \in X, x \neq y} \frac{|f(x)-f(y)|}{d(x, y)^{\alpha}} .
$$

For $\alpha \in] 0,1$, we say that the geodesic flow on $T^{1} M$ is exponentially mixing for the Hölder regularity $\alpha$ if there exist $c, \kappa>0$ such that for all $\phi, \psi \in \mathscr{C}_{\mathrm{c}}^{\alpha}\left(T^{1} M\right)$ and $t \in \mathbb{R}$, we have

$$
\left|\int_{T^{1} M} \phi \circ g^{-t} \psi d \overline{m_{\mathrm{BM}}}-\int_{T^{1} M} \phi d \overline{m_{\mathrm{BM}}} \int_{T^{1} M} \psi d \overline{m_{\mathrm{BM}}}\right| \leq c e^{-\kappa|t|}\|\phi\|_{\alpha}\|\psi\|_{\alpha} .
$$


This holds for compact manifolds $M$ when $M$ is locally symmetric by [Moo, when $M$ is two-dimensional by [Dol], and when $M$ is 1/9-pinched by [GLP, Coro. 2.7], see also [MO].

Theorem 15 Let $\widetilde{M}$ be a complete simply connected Riemannian manifold with sectional curvature at most -1 . Let $\Gamma$ be a nonelementary discrete group of isometries of $\widetilde{M}$ and let $M=\Gamma \backslash \widetilde{M}$. Assume that $(\widetilde{M}, \Gamma)$ has radius-Hölder-continuous strong stable/unstable ball masses. Let $\mathscr{D}^{-}=\left(D_{i}^{-}\right)_{i \in I^{-}}$and $\mathscr{D}^{+}=\left(D_{j}^{+}\right)_{j \in I^{+}}$be locally finite $\Gamma$-equivariant families of nonempty proper closed convex subsets of $\widetilde{M}$ with finite nonzero skinning measure.

(1) Assume that $M$ is compact and that the geodesic flow on $T^{1} M$ is mixing with exponential speed for the Hölder regularity. Then there exist $\alpha \in] 0,1\left[\right.$ and $\kappa^{\prime}>0$ such that for all nonnegative $\psi^{ \pm} \in \mathscr{C}_{\mathrm{c}}^{\alpha}\left(T^{1} M\right)$, we have, as $t \rightarrow+\infty$,

$$
\begin{aligned}
\frac{\delta\left\|m_{\mathrm{BM}}\right\|}{e^{\delta t}} \sum_{v, w \in T^{1} M} m_{\partial_{+}^{1} \mathscr{D}^{-}}(v) & m_{\partial_{-}^{1} \mathscr{D}^{+}}(w) n_{t}(v, w) \psi^{-}(v) \psi^{+}(w) \\
= & \int_{T^{1} M} \psi^{-} d \sigma_{\mathscr{D}^{-}}^{+} \int_{T^{1} M} \psi^{+} d \sigma_{\mathscr{D}^{+}}^{-}+\mathrm{O}\left(e^{-\kappa^{\prime} t}\left\|\psi^{-}\right\|_{\alpha}\left\|\psi^{+}\right\|_{\alpha}\right) .
\end{aligned}
$$

(2) Assume that $\widetilde{M}$ is a symmetric space, that $D_{k}^{ \pm}$has smooth boundary for every $k \in I^{ \pm}$, that $M$ has finite volume, and that the geodesic flow on $T^{1} M$ is mixing with exponential speed for the Sobolev regularity. Then there exist $\ell \in \mathbb{N}$ and $\kappa^{\prime}>0$ such that for all nonnegative maps $\psi^{ \pm} \in \mathscr{C}_{\mathrm{c}}^{\ell}\left(T^{1} M\right)$, we have, as $t \rightarrow+\infty$,

$$
\begin{aligned}
\frac{\delta\left\|m_{\mathrm{BM}}\right\|}{e^{\delta t}} \sum_{v, w \in T^{1} M} m_{\partial_{+}^{1} \mathscr{D}^{-}}(v) & m_{\partial_{-}^{1} \mathscr{D}+}(w) n_{t}(v, w) \psi^{-}(v) \psi^{+}(w) \\
= & \int_{T^{1} M} \psi^{-} d \sigma_{\mathscr{D}^{-}}^{+} \int_{T^{1} M} \psi^{+} d \sigma_{\mathscr{D}^{+}}^{-}+\mathrm{O}\left(e^{-\kappa^{\prime} t}\left\|\psi^{-}\right\|_{\ell}\left\|\psi^{+}\right\|_{\ell}\right) .
\end{aligned}
$$

Furthermore, if $\mathscr{D}^{-}$and $\mathscr{D}^{+}$respectively have nonzero finite outer and inner skinning measures, if $(\widetilde{M}, \Gamma)$ satisfies condition (1) or (2) above, then there exists $\kappa^{\prime \prime}>0$ such that, as $t \rightarrow+\infty$,

$$
\mathscr{N}_{\mathscr{D}^{-}, \mathscr{D}^{+}}(t)=\frac{\left\|\sigma_{\mathscr{D}^{-}}^{+}\right\|\left\|\sigma_{\mathscr{D}^{+}}^{-}\right\|}{\delta\left\|m_{\mathrm{BM}}\right\|} e^{\delta t}\left(1+\mathrm{O}\left(e^{-\kappa^{\prime \prime} t}\right)\right)
$$

The maps $\mathrm{O}(\cdot)$ depend on $\widetilde{M}, \Gamma, \mathscr{D}$, and the speeds of mixing.

Proof. We follow the proofs of Theorem 8 and Corollary 12, adding a regularisation of the test functions $\widetilde{\phi}_{\eta}^{ \pm}$as for the deduction of [PP5, Theo. 20] from [PP5, Theo. 19].

Let $\beta$ be either $\alpha \in] 0,1]$ in the Hölder regularity case or $\ell \in \mathbb{N}$ in the Sobolev regularity case. We fix $i \in I^{-}, j \in I^{+}$, and we use the notation of Equation (13). Let $\widetilde{\psi}^{ \pm} \in \mathscr{C}^{\beta}\left(\partial_{\mp}^{1} D^{ \pm}\right)$be such that $\int_{T^{1}} \widetilde{M}^{ \pm} \widetilde{\psi}^{ \pm} \widetilde{\sigma}_{\mathscr{D}}^{\mp}$ is finite. Under the assumptions of Assertion (1) or (2), we first prove the following avatar of Equation (14), indicating only the required changes in its proof: there exists $\kappa_{0}>0$ (independent of $\psi^{ \pm}$) such that, as $T \rightarrow+\infty$,

$$
\begin{aligned}
\delta\left\|m_{\mathrm{BM}}\right\| e^{-\delta T} \sum_{\gamma \in \Gamma, 0<\ell_{\gamma} \leq T} \widetilde{\psi}^{-}\left(v_{\gamma}^{-}\right) \widetilde{\psi}^{+}\left(v_{\gamma}^{+}\right) \\
=\int_{\partial_{+}^{1} D^{-}} \widetilde{\psi}^{-} d \widetilde{\sigma}^{+} \int_{\partial_{-}^{1} D^{+}} \widetilde{\psi}^{+} d \widetilde{\sigma}^{-}+\mathrm{O}\left(e^{-\kappa_{0} T}\left\|\widetilde{\psi}^{-}\right\|_{\beta}\left\|\widetilde{\psi}^{+}\right\|_{\beta}\right) .
\end{aligned}
$$


By Lemma 6 and the Hölder regularity of the strong stable and unstable foliations under the assumptions of Assertion (1), or by the smoothness of the boundary of $D^{ \pm}$under the assumptions of Assertion (2), the maps $f_{D^{\mp}}^{ \pm}: \mathscr{V}_{\eta, R}^{ \pm}\left(\partial_{ \pm}^{1} D^{\mp}\right) \rightarrow \partial_{ \pm}^{1} D^{\mp}$ are respectively Hölder-continuous or smooth fibrations, whose fiber over $w \in \partial_{ \pm}^{1} D^{\mp}$ is exactly $V_{w, \eta, R}^{ \pm}$. By applying leafwise the regularisation process described in the proof of [PP5, Theo. 20] to characteristic functions, there exists a constant $\kappa_{1}>0$ and $\chi_{\eta, R}^{ \pm} \in \mathscr{C}^{\beta}\left(T^{1} \widetilde{M}\right)$ such that

- $\left\|\chi_{\eta, R}^{ \pm}\right\|_{\beta}=\mathrm{O}\left(\eta^{-\kappa_{1}}\right)$,

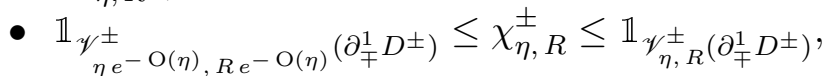

- for every $w \in \partial_{\mp}^{1} D^{ \pm}$, we have

$$
\int_{\mathscr{V}_{w, \eta, R}^{\mp}} \chi_{\eta, R}^{ \pm} d \nu_{w}^{ \pm}=\nu_{w}^{ \pm}\left(\mathscr{V}_{w, \eta, R}^{\mp}\right) e^{-\mathrm{O}(\eta)}=\nu_{w}^{ \pm}\left(\mathscr{V}_{w, \eta e^{-\mathrm{O}(\eta)}}^{\mp}, R e^{-\mathrm{O}(\eta)}\right) e^{\mathrm{O}(\eta)}
$$

where the measures $\nu_{w}^{ \pm}$on $W^{0 \mp}(v) \simeq \mathbb{R} \times W^{\mp}(v)$ are defined by $d \nu_{w}^{+}=d s d \mu_{W^{-}(w)}$ and $d \nu_{w}^{-}=d s d \mu_{W^{+}(w)}$. We now define the new test functions. For every $w \in \partial_{\mp}^{1} D^{ \pm}$, let

$$
H_{\eta, R}^{ \pm}(w)=\frac{1}{\int_{\mathscr{V}_{w, \eta, R}^{\mp}}^{\mp} \chi_{\eta, R}^{ \pm} d \nu_{w}^{ \pm}}
$$

Let $\Phi_{\eta}^{ \pm}: T^{1} \widetilde{M} \rightarrow \mathbb{R}$ be the map defined by

$$
\Phi_{\eta}^{ \pm}=\left(H_{\eta, R}^{ \pm} \widetilde{\psi}^{ \pm}\right) \circ f_{D^{ \pm}}^{\mp} \chi_{\eta, R}^{ \pm} .
$$

The support of this map is contained in $\mathscr{V}_{\eta, R}^{ \pm}\left(\partial_{\mp}^{1} D^{ \pm}\right)$. Since $M$ is compact in Assertion (1) and by homogeneity in Assertion (2), if $R$ is large enough, by the definitions of the measures $\nu_{w}^{ \pm}$, the denominator of $H_{\eta, R}^{ \pm}(w)$ is a least $c \eta$, where $c>0$. The map $H_{\eta, R}^{ \pm}$is hence Hölder continuous under the assumptions of Assertion (1), and is smooth under the ones of Assertion (2). Therefore $\Phi_{\eta}^{ \pm} \in \mathscr{C}^{\beta}\left(T^{1} \widetilde{M}\right)$ and there exists $\kappa_{2}>0$ such that

$$
\left\|\Phi_{\eta}^{ \pm}\right\|_{\beta}=\mathrm{O}\left(\eta^{-\kappa_{2}}\left\|\widetilde{\psi}^{ \pm}\right\|_{\beta}\right)
$$

The functions $\Phi_{\eta}^{\mp}$ are measurable, nonnegative and satisfy

$$
\int_{T^{1} \widetilde{M}} \Phi_{\eta}^{\mp} d \widetilde{m}_{\mathrm{BM}}=\int_{\partial_{\mp}^{1} D^{ \pm}} \widetilde{\psi}^{ \pm} d \widetilde{\sigma}^{\mp} .
$$

As in the second step of the proof of Theorem 8, we will estimate in two ways the quantity

$$
I_{\eta}(T)=\int_{0}^{T} e^{\delta t} \sum_{\gamma \in \Gamma} \int_{T^{1} \widetilde{M}}\left(\Phi_{\eta}^{-} \circ g^{-t / 2}\right)\left(\Phi_{\eta}^{+} \circ g^{t / 2} \circ \gamma^{-1}\right) d \widetilde{m}_{\mathrm{BM}} d t
$$

We first apply the mixing property, now with exponential decay of correlations, as in the third step of the proof of Theorem 8 . For all $t \geq 0$, let

$$
A_{\eta}(t)=\sum_{\gamma \in \Gamma} \int_{v \in T^{1} \widetilde{M}} \Phi_{\eta}^{-}\left(g^{-t / 2} v\right) \Phi_{\eta}^{+}\left(g^{t / 2} \gamma^{-1} v\right) d \widetilde{m}_{\mathrm{BM}}(v)
$$


Then with $\kappa>0$ as in the definitions of the exponential mixing, we have

$$
\begin{aligned}
A_{\eta}(t) & =\frac{1}{\left\|m_{\mathrm{BM}}\right\|} \int_{T^{1} \widetilde{M}} \Phi_{\eta}^{-} d \widetilde{m}_{\mathrm{BM}} \int_{T^{1} \widetilde{M}} \Phi_{\eta}^{+} d \widetilde{m}_{\mathrm{BM}}+\mathrm{O}\left(e^{-\kappa t}\left\|\Phi_{\eta}^{-}\right\|_{\beta}\left\|\Phi_{\eta}^{+}\right\|_{\beta}\right) \\
& =\frac{1}{\left\|m_{\mathrm{BM}}\right\|} \int_{\partial_{+}^{1} D^{-}} \widetilde{\psi}^{-} d \widetilde{\sigma}^{+} \int_{\partial_{-}^{1} D^{+}} \widetilde{\psi}^{+} d \widetilde{\sigma}^{-}+\mathrm{O}\left(e^{-\kappa t} \eta^{-2 \kappa_{2}}\left\|\widetilde{\psi}^{-}\right\|_{\beta}\left\|\widetilde{\psi}^{+}\right\|_{\beta}\right) .
\end{aligned}
$$

Hence by integrating,

$$
I_{\eta}(T)=\frac{e^{\delta T}}{\delta\left\|m_{\mathrm{BM}}\right\|}\left(\int_{\partial_{+}^{1} D^{-}} \widetilde{\psi}^{-} d \widetilde{\sigma}^{+} \int_{\partial_{-}^{1} D^{+}} \widetilde{\psi}^{+} d \widetilde{\sigma}^{-}+\mathrm{O}\left(e^{-\kappa T} \eta^{-2 \kappa_{2}}\left\|\widetilde{\psi}^{-}\right\|_{\beta}\left\|\widetilde{\psi}^{+}\right\|_{\beta}\right)\right) .
$$

Now, we exchange the integral over $t$ and the summation over $\gamma$ in the definition of $I_{\eta}(T)$, and we proceed as in the fourth step of the proof of Theorem 8 ;

$$
I_{\eta}(T)=\sum_{\gamma \in \Gamma} \int_{0}^{T} e^{\delta t} \int_{T^{1} \widetilde{M}}\left(\Phi_{\eta}^{-} \circ g^{-t / 2}\right)\left(\Phi_{\eta}^{+} \circ g^{t / 2} \circ \gamma^{-1}\right) d \widetilde{m}_{\mathrm{BM}} d t .
$$

Let $\widehat{\Phi}_{\eta}^{ \pm}=H_{\eta, R}^{ \pm} \circ f_{D^{ \pm}}^{\mp} \chi_{\eta, R}^{ \pm}$, so that $\Phi_{\eta}^{ \pm}=\widetilde{\psi}^{ \pm} \circ f_{D^{ \pm}}^{\mp} \widehat{\Phi}_{\eta}^{ \pm}$. By the last two properties of the regularised maps $\chi_{\eta, R}^{ \pm}$, we have, with $\phi_{\eta}^{\mp}$ defined as in Equation (16),

$$
\phi_{\eta e^{-\mathrm{O}(\eta)}, R e^{-\mathrm{O}(\eta), \partial_{\mp}^{1} D^{ \pm}}}^{ \pm} e^{-\mathrm{O}(\eta)} \leq \widehat{\Phi}_{\eta}^{ \pm} \leq \phi_{\eta}^{ \pm} e^{\mathrm{O}(\eta)} .
$$

If $v \in T^{1} \widetilde{M}$ belongs to the support of $\left(\Phi_{\eta}^{-} \circ g^{-t / 2}\right)\left(\Phi_{\eta}^{+} \circ g^{t / 2} \circ \gamma^{-1}\right)$, then we have $v \in g^{t / 2} \mathscr{V}_{\eta, R}^{+}\left(\partial_{+}^{1} D^{-}\right) \cap g^{-t / 2 \mathscr{V}_{\eta, R}^{-}}\left(\gamma \partial_{-}^{1} D^{+}\right)$. Hence the properties (i), (ii) and (iii) of the fourth step of the proof of Theorem 8 still hold (with $\Omega_{-}=\partial_{+}^{1} D^{-}$and $\Omega_{+}=\partial_{-}^{1}\left(\gamma D^{+}\right)$). In particular, if $w^{-}=f_{D^{-}}^{+}(v)$ and $w^{+}=f_{\gamma D^{+}}^{-}(v)$, we have, as in the fifth step of the proof of Theorem 8, that

$$
d\left(w^{ \pm}, v_{\gamma}^{ \pm}\right)=\mathrm{O}\left(\eta+e^{-\ell_{\gamma} / 2}\right) .
$$

Hence, with $\kappa_{3}=\alpha$ in the Hölder case and $\kappa_{3}=1$ in the Sobolev case (we may assume that $\ell \geq 1$ ), we have

$$
\left|\widetilde{\psi}^{ \pm}\left(w^{ \pm}\right)-\widetilde{\psi}^{ \pm}\left(v_{\gamma}^{ \pm}\right)\right|=\mathrm{O}\left(\left(\eta+e^{-\ell_{\gamma} / 2}\right)^{\kappa_{3}}\left\|\widetilde{\psi}^{ \pm}\right\|_{\beta}\right)
$$

Therefore there exists a constant $\kappa_{4}>0$ such that

$$
\begin{array}{r}
I_{\eta}(T)=\sum_{\gamma \in \Gamma}\left(\widetilde{\psi}^{-}\left(v_{\gamma}^{-}\right) \widetilde{\psi}^{+}\left(v_{\gamma}^{+}\right)+\mathrm{O}\left(\left(\eta+e^{-\ell_{\gamma} / 2}\right)^{\kappa_{4}}\left\|\widetilde{\psi}^{-}\right\|_{\beta}\left\|\widetilde{\psi}^{+}\right\|_{\beta}\right)\right) \times \\
\int_{0}^{T} e^{\delta t} \int_{v \in T^{1}} \widetilde{M} \widehat{\Phi}_{\eta}^{-}\left(g^{-t / 2} v\right) \widehat{\Phi}_{\eta}^{+}\left(\gamma^{-1} g^{t / 2} v\right) d \widetilde{m}_{\mathrm{BM}}(v) d t .
\end{array}
$$

Now, using the inequalities (31), Equation 28 follows as in the last two steps of the proof of Theorem 8 , by taking $\eta=e^{-\kappa_{5} T}$ for some $\kappa_{5}>0$.

In the same way that Corollary 12 is deduced from Theorem 8 , the following result can be deduced from Equation (28) under the assumptions of assertions (1) or (2). Let $\left(\widetilde{\psi}_{k}^{ \pm}\right)_{k \in I^{ \pm}}$be a $\Gamma$-equivariant family of nonnegative maps $\widetilde{\psi}_{k}^{ \pm} \in \mathscr{C}^{\beta}\left(\partial_{\mp}^{1} D_{k}^{ \pm}\right)$such that $\sup _{k \in I^{ \pm}}\left\|\widetilde{\psi}_{k}^{ \pm}\right\|_{\beta}$ is finite. Extend $\widetilde{\psi}_{k}^{ \pm}$by 0 outside $\partial_{\mp}^{1} D_{k}^{ \pm}$to define a function on $T^{1} \widetilde{M}$. 
The measurable $\Gamma$-invariant function $\widetilde{\Psi}^{ \pm}=\sum_{k \in I^{ \pm} / \sim} \widetilde{\psi}_{k}^{ \pm}$on $T^{1} \widetilde{M}$ defines a measurable function $\Psi^{ \pm}$on $T^{1} M$. Assume that $\int_{T^{1} M} \Psi^{ \pm} d \sigma_{\mathscr{D}^{ \pm}}^{\mp}$ is finite. Then there exists $\kappa_{0}^{\prime}>0$ (independent of $\left.\left(\widetilde{\psi}_{k}^{ \pm}\right)_{k \in I^{ \pm}}\right)$such that, as $t \rightarrow+\infty$,

$$
\begin{aligned}
& \delta\left\|m_{\mathrm{BM}}\right\| e^{-\delta t} \sum_{v, w \in T^{1} M} m_{\partial_{+}^{1} \mathscr{D}^{-}}(v) m_{\partial_{-}^{1} \mathscr{D}^{+}}(w) n_{t}(v, w) \Psi^{-}(v) \Psi^{+}(w) \\
= & \int_{T^{1} M} \Psi^{-} d \sigma_{\mathscr{D}^{-}}^{+} \int_{T^{1} M} \Psi^{+} d \sigma_{\mathscr{D}^{+}}^{-}+\mathrm{O}\left(e^{-\kappa_{0}^{\prime} T} \sup _{i \in I^{-}}\left\|\widetilde{\psi}_{i}^{-}\right\|_{\beta} \sup _{j \in I^{+}}\left\|\widetilde{\psi}_{j}^{+}\right\|_{\beta}\right) .
\end{aligned}
$$

Now to prove the assertions (1) and (2), we proceed as follows. If $\psi^{ \pm} \in \mathscr{C}_{c}^{\beta}\left(T^{1} M\right)$, for every $k \in I^{ \pm}$, we denote by $\widetilde{\psi}_{k}^{ \pm}$the restriction to $\partial_{\mp}^{1} D_{k}^{ \pm}$of $\psi^{ \pm} \circ T p$ where $p: \widetilde{M} \rightarrow M$ is the universal cover. Note that the map $\Psi^{ \pm}$defined above coincides with $\psi^{ \pm}$on the elements $u \in T^{1} M$ such that $m_{\mathscr{D}^{ \pm}}(u) \neq 0$, and that $\sup _{k \in I^{ \pm}}\left\|\widetilde{\psi}_{k}^{ \pm}\right\|_{\beta} \leq\left\|\psi^{ \pm}\right\|_{\beta}$. Hence the assertions (1) and (2) follow from Equation (32).

The last statement of Theorem 15 follows by taking as the functions $\widetilde{\psi}_{k}^{ \pm}$the constant functions 1 in Equation (32).

\section{Counting closed subsets of limit sets}

In this section, we give counting asymptotics on very general equivariant families of subsets of the limit sets of discrete groups of isometries of rank one symmetric spaces.

Recall that the rank 1 symmetric spaces are the hyperbolic spaces $\mathbb{H}_{\mathbb{F}}^{n}$ where $\mathbb{F}$ is the set $\mathbb{R}$ of real numbers, $\mathbb{C}$ of complex numbers, $\mathbb{H}$ of Hamilton's quaternions, or $\mathbb{O}$ of octonions, and $n \geq 2$, with $n=2$ if $\mathbb{K}=\mathbb{O}$. We will normalise them so that their maximal sectional curvature is -1 . We denote the convex hull in $\mathbb{H}_{\mathbb{F}}^{n}$ of any subset $A$ of $\mathbb{H}_{\mathbb{F}}^{n} \cup \partial_{\infty} \mathbb{H}_{\mathbb{F}}^{n}$ by $\mathscr{C} A$.

We start with $\mathbb{H}_{\mathbb{R}}^{n}$. The Euclidean diameter of a subset $A$ of the Euclidean space $\mathbb{R}^{n-1}$ is denoted by $\operatorname{diam} A$. For any nonempty subset $B$ of the standard sphere $\mathbb{S}^{n-1}$, we denote by $\theta(B)$ the least upper bound of half the visual angle over pairs of points in $B$ seen from the center of the sphere. Let $\mathscr{H}_{\infty}$ be the horoball in $\mathbb{H}_{\mathbb{R}}^{n}$ centred at $\infty$, consisting of the points with vertical coordinates at least 1 . For every Patterson-Sullivan density $\left(\mu_{x}\right)_{x \in \widetilde{M}}$ for a discrete nonelementary group of isometries $\Gamma$ of $\widetilde{M}$, for every horoball $\mathscr{H}$ in $\widetilde{M}$, and for every geodesic ray $\rho$ starting from a point of $\partial \mathscr{H}$ and converging to the point at infinity $\xi$ of $\mathscr{H}$, the measure $e^{\delta t} \mu_{\rho(t)}$ converges as $t$ tends to $+\infty$ to a measure $\mu_{\mathscr{H}}$ on $\partial_{\infty} \widetilde{M}-\{\xi\}$, independent on the choice of $\rho$ (see [HP2, $\left.\S 2\right]$ ).

Corollary 16 Let $\Gamma$ be a discrete nonelementary group of isometries of $\mathbb{H}_{\mathbb{R}}^{n}$, with finite Bowen-Margulis measure $m_{\mathrm{BM}}$. Let $\left(F_{i}\right)_{i \in I}$ be a $\Gamma$-equivariant family of nonempty closed subsets in the limit set $\Lambda \Gamma$, whose family $\mathscr{D}^{+}=\left(\mathscr{C} F_{i}\right)_{i \in I}$ of convex hulls in $\mathbb{H}_{\mathbb{R}}^{n}$ is locally finite, with finite nonzero skinning measure.

(1) In the upper halfspace model of $\mathbb{H}_{\mathbb{R}}^{n}$, assume that $\Lambda \Gamma$ is bounded in $\mathbb{R}^{n-1}=\partial_{\infty} \mathbb{H}_{\mathbb{R}}^{n}-\{\infty\}$, and that $\infty$ is not the fixed point of an elliptic element of $\Gamma$. Let $\mathscr{D}^{-}$be the $\Gamma$-equivariant family $\left(\gamma \mathscr{H}_{\infty}\right)_{\gamma \in \Gamma}$. Then, as $T \rightarrow+\infty$,

$$
\operatorname{Card}\left\{i \in I / \sim: \operatorname{diam}\left(F_{i}\right) \geq 1 / T\right\} \sim \frac{\left\|\sigma_{\mathscr{D}^{-}}\right\|\left\|\sigma_{\mathscr{D}^{+}}\right\|}{\delta\left\|m_{\mathrm{BM}}\right\|}(2 T)^{\delta} .
$$


(2) In the unit ball model of $\mathbb{H}_{\mathbb{R}}^{n}$, assume that no nontrivial element of $\Gamma$ fixes 0 . As $T \rightarrow+\infty$, we have

$$
\operatorname{Card}\left\{i \in I / \sim: \cot \theta\left(F_{i}\right)<T\right\} \sim \frac{\left\|\mu_{0}\right\|\left\|\sigma_{\mathscr{D}}\right\|}{\delta\left\|m_{\mathrm{BM}}\right\|}(2 T)^{\delta} .
$$

(3) In the upper halfspace model of $\mathbb{H}_{\mathbb{R}}^{n}$, assume that $\infty$ is not the fixed point of an elliptic element of $\Gamma$. Let $\Omega$ be a Borel subset of $\mathbb{R}^{n-1}=\partial_{\infty} \mathbb{H}_{\mathbb{R}}^{n}-\{\infty\}$ such that $\mu_{\mathscr{H}_{\infty}}(\Omega)$ is finite and positive and $\mu_{\mathscr{H}_{\infty}}(\partial \Omega)=0$. Then, as $T \rightarrow+\infty$,

$$
\operatorname{Card}\left\{i \in I / \sim: \operatorname{diam}\left(F_{i}\right) \geq 1 / T, F_{i} \cap \Omega \neq \emptyset\right\} \sim \frac{\mu_{\mathscr{H}_{\infty}}(\Omega)\left\|\sigma_{\mathscr{D}^{+}}\right\|}{\delta\left\|m_{\mathrm{BM}}\right\|}(2 T)^{\delta} .
$$

This corollary generalises OS1, Thm. 1.4] and OS3, Thm. 1.2]) where the subsets $F_{i}$ are round spheres. When $\Gamma$ is an arithmetic lattice, the error term in the claims (1) and (2) is $\mathrm{O}\left(T^{\delta-\kappa}\right)$ for some $\kappa>0$, as it follows from Theorem 15 (2) (using the Riemannian convolution smoothing process of Green and $\mathrm{Wu}$ as in [PP4, $\S 3$ to smooth by a very small perturbation the boundary of $\mathscr{C} F_{i}$, so that the perturbation of the lengths of the common perpendiculars are uniformly small).

Proof. As the Bowen-Margulis measure is finite in a locally symmetric space, the geodesic flow is mixing (see for instance [Dal2, p. 982]).

(1) The skinning measure $\sigma_{\mathscr{D}^{-}}^{+}$is nonzero since $\Gamma$ is nonelementary, and finite since the support of $\widetilde{\sigma}_{\mathscr{H}_{\infty}}^{+}$, consisting of the points $v \in \partial_{+}^{1} \mathscr{H}_{\infty}$ such that $v_{+} \in \Lambda \Gamma$, is compact.

For each $i \in I$, let $x_{i}, y_{i} \in F_{i}$ be such that $\operatorname{diam} F_{i}=\left\|x_{i}-y_{i}\right\|$, where $\|\cdot\|$ is the Euclidean norm in $\mathbb{R}^{n-1}$. The (signed) length $\ell\left(\alpha_{e, i}\right)$ of the common perpendicular $\alpha_{e, i}$ from $\mathscr{H}_{\infty}$ to the geodesic line in $\mathbb{H}_{\mathbb{R}}^{n}$ with endpoints $x_{i}$ and $y_{i}$ (which is also the common perpendicular from $\mathscr{H}_{\infty}$ to $\left.\mathscr{C} F_{i}\right)$ is $\log \frac{2}{\left\|x_{i}-y_{i}\right\|}$. Thus, since the stabiliser in $\Gamma$ of an element of $\partial_{+}^{1} \mathscr{H}_{\infty}$ is trivial, and since $\Gamma$ acts transitively on the index set of the family $\mathscr{D}^{-}$,

$$
\begin{aligned}
\operatorname{Card}\left\{i \in I / \sim: \operatorname{diam}\left(F_{i}\right) \geq 1 / T\right\} & =\operatorname{Card}\left\{i \in I / \sim: \ell\left(\alpha_{e, i}\right) \leq \log (2 T)\right\} \\
& =\mathscr{N}_{\mathscr{D}^{-}, \mathscr{D}^{+}}(\log (2 T))
\end{aligned}
$$

which implies the claim (1) by Corollary 12 .

(2) Let $\mathscr{D}^{-}$be the $\Gamma$-equivariant family $(\{\gamma 0\})_{\gamma \in \Gamma}$, whose skinning measure in $T^{1} \widetilde{M}$ is $\widetilde{\sigma}_{\mathscr{D}^{-}}^{+}=\sum_{\gamma \in \Gamma} \mu_{\gamma 0}$, so that $\left\|\sigma_{\mathscr{D}^{-}}^{+}\right\|$is equal to the (finite and nonzero) total mass $\left\|\mu_{0}\right\|$ of the Patterson-Sullivan measure at 0 , since the stabiliser of 0 in $\Gamma$ is trivial.

For each $i \in I$, let $x_{i}, y_{i} \in F_{i}$ be such that $\theta\left(F_{i}\right)=\theta\left(\left\{x_{i}, y_{i}\right\}\right)$. The angle of parallelism formula (see for instance [Bea, p. 147]) implies that $\cot \theta\left(F_{i}\right)=\sinh d\left(0, \mathscr{C} F_{i}\right)$, and the rest of the proof is analogous to that of claim (1).

(3) Note that we do not assume in (3) that the $\Gamma$-equivariant family $\mathscr{D}^{-}=\left(\gamma \mathscr{H}_{\infty}\right)_{\gamma \in \Gamma}$ is locally finite, and we will only use Equation (14) (and not Corollary 12) to prove the claim (3). One can check that the proof of Equation (14) does not use the local finiteness property of $\mathscr{D}^{-}$. By the definition of the skinning measure $\widetilde{\sigma}_{\mathscr{H}}^{+}$with base point $x_{0}=\rho(t)$ where $\rho$ is a geodesic ray from a point of $\partial \mathscr{H}_{\infty}$ to $\infty$, and letting $t \rightarrow+\infty$, we see that the pushforward of the measure $\mu_{\mathscr{H}_{\infty}}$ by the map $x \mapsto(0,-1) \in T_{(x, 1)}^{1} \mathbb{H}_{\mathbb{R}}^{n}$ from $\mathbb{R}^{n-1}$ to $\partial_{+}^{1} \mathscr{H}_{\infty}$ is exactly the skinning measure $\widetilde{\sigma}_{\mathscr{H}}^{+}$. If diam $F_{i}$ is small and $F_{i}$ meets $\Omega$, then $F_{i}$ is contained in $\mathscr{N}_{\epsilon} \Omega$ for some small $\epsilon>0$, and $\mu_{\mathscr{H}_{\infty}}\left(\mathscr{N}_{\epsilon} \Omega\right)$ converges to $\mu_{\mathscr{H}_{\infty}}(\Omega)$ as $\epsilon \rightarrow 0$. We hence apply Equation (14) with $\Omega_{e}^{-}$the image of $\Omega$ by this map $x \mapsto(0,-1)$. 
Corollary 4 in the Introduction is a special case of the following corollary. For every parabolic fixed point $p$ of a discrete isometry group $\Gamma$ of $\mathbb{H}_{\mathbb{R}}^{n}$, recall that, by Bieberbach's theorem, the stabiliser of $p$ in $\Gamma$ contains a subgroup isomorphic to $\mathbb{Z}^{k}$ with finite index, and $k=\operatorname{rk}_{\Gamma}(p) \geq 1$ is called the rank of $p$ in $\Gamma$.

Corollary 17 Let $\Gamma$ be a geometrically finite discrete group of isometries of the upper halfspace model of $\mathbb{H}_{\mathbb{R}}^{n}$, whose limit set $\Lambda \Gamma$ is bounded in $\mathbb{R}^{n-1}=\partial_{\infty} \mathbb{H}_{\mathbb{R}}^{n}-\{\infty\}$. Let $\Gamma_{0}$ be a geometrically finite subgroup of $\Gamma$ with infinite index. Assume that the Hausdorff dimension $\delta$ of $\Lambda \Gamma$ is bigger than $\operatorname{rk}_{\Gamma}(p)-\operatorname{rk}_{\Gamma_{0}}(p)$ for every parabolic fixed point $p$ of $\Gamma_{0}$. Then, there exists an explicitable $c>0$ such that, as $T \rightarrow+\infty$,

$$
\operatorname{Card}\left\{\gamma \in \Gamma / \Gamma_{0}: \operatorname{diam}\left(\gamma \Lambda \Gamma_{0}\right) \geq 1 / T\right\} \sim c T^{\delta} .
$$

The assumption on the ranks of parabolic groups (needed to apply [PP5, Theo. 10]) is in particular satisfied if every maximal parabolic sugbroup of $\Gamma_{0}$ has finite index in the maximal parabolic subgroup of $\Gamma$ containing it, as well as when $n=3$ and $\delta>1$ (or equivalently if $\Gamma$ does not contain a Fuchsian group with index at most 2, when $\Lambda \Gamma$ is not totally disconnected, see [CaT, Theo. $3(3)])$.

Proof. First assume that $\infty$ is not fixed by an elliptic element of $\Gamma$. Since $\Gamma$ is geometrically finite, its Bowen-Margulis measure is finite (see for instance [DOP]). The critical exponent of $\Gamma$ is equal to the Hausdorff dimension $\delta$ of $\Lambda \Gamma$. Let $\Gamma_{0}^{\prime}$ be the stabiliser of the limit set $\Lambda \Gamma_{0}$ of $\Gamma_{0}$, and recall that $\Gamma_{0}$ has finite index in $\Gamma_{0}^{\prime}$ (see for instance Kap, Coro. 4.136]). Let us consider $I=\Gamma$, the family $\left(F_{i}=i \Lambda \Gamma_{0}\right)_{i \in I}$ (which consists of nonempty closed subsets of $\Lambda \Gamma$ ), and $\mathscr{D}^{+}=\left(\mathscr{C} F_{i}\right)_{i \in I}$ (which is locally finite), so that $I / \sim=\Gamma / \Gamma_{0}^{\prime}$. Since $\Gamma_{0}$ is geometrically finite, the convex set $\mathscr{C} \Lambda \Gamma_{0}$ is almost cone-like in cusps and any parabolic subgroup of $\Gamma$ has regular growth (see the definitions in PP5, Sect. 4]). Hence, under the hypothesis on the ranks of parabolic groups, by [PP5, Theo. 10], the skinning measure $\sigma_{\mathscr{D}+}^{-}$is finite. It is nonzero by [PP5, Prop. 4 (iv)], since $\Lambda \Gamma_{0} \neq \Lambda \Gamma$ as $\Gamma_{0}$ has infinite index (as seen above). Note that

$$
\begin{aligned}
\operatorname{Card}\left\{\gamma \in \Gamma / \Gamma_{0}: \operatorname{diam}\left(\gamma \Lambda \Gamma_{0}\right) \geq\right. & 1 / T\} \\
& =\left[\Gamma_{0}^{\prime}: \Gamma_{0}\right] \operatorname{Card}\left\{\gamma \in \Gamma / \Gamma_{0}^{\prime}: \operatorname{diam}\left(\gamma \Lambda \Gamma_{0}\right) \geq 1 / T\right\} .
\end{aligned}
$$

The result then follows from Corollary 16 (1).

If $\infty$ is fixed by an elliptic element of $\Gamma$, let $\Gamma^{\prime}$ be a finite-index torsion-free subgroup of $\Gamma$ (in particular $\Gamma^{\prime}$ is geometrically finite and $\Lambda \Gamma^{\prime}=\Lambda \Gamma$ ). The action by left translations of $\Gamma^{\prime}$ on $\Gamma / \Gamma_{0}$ has finitely many (pairwise distinct) orbits, say $\alpha_{1} \Gamma_{0}, \ldots, \alpha_{k} \Gamma_{0}$. For $i=$ $1, \ldots, k$, the group $\Gamma_{i}^{\prime}=\alpha_{i} \Gamma_{0} \alpha_{i}^{-1} \cap \Gamma^{\prime}$ is geometrically finite with infinite index in $\Gamma^{\prime}$. Let $A(T)=\left\{\gamma \Gamma_{0} \in \Gamma / \Gamma_{0}: \operatorname{diam}\left(\gamma \Lambda \Gamma_{0}\right) \geq 1 / T\right\}$ and $A_{i}(T)=\left\{\gamma \Gamma_{0} \in A(T): \Gamma^{\prime} \gamma \Gamma_{0}=\Gamma^{\prime} \alpha_{i} \Gamma_{0}\right\}$ for $i=1, \ldots, k$, so that Card $A(T)=\sum_{i=1}^{k} \operatorname{Card} A_{i}(T)$. The map $\gamma \Gamma_{0} \rightarrow \gamma^{\prime} \Gamma_{i}^{\prime}$ from $A_{i}(T)$ to $\left\{\gamma^{\prime} \Gamma_{i}^{\prime} \in \Gamma^{\prime} / \Gamma_{i}^{\prime}: \operatorname{diam}\left(\gamma^{\prime} \Lambda \Gamma_{i}^{\prime}\right) \geq 1 / T\right\}$ where $\gamma^{\prime} \in \Gamma^{\prime}$ satisfies $\gamma \Gamma_{0}=\gamma^{\prime} \alpha_{i} \Gamma_{0}$ is easily seen to be well-defined and a bijection. Note that the Hausdorff dimension $\delta$ of $\Lambda \Gamma^{\prime}=\Lambda \Gamma$ is bigger than $\operatorname{rk}_{\Gamma^{\prime}}(p)-\operatorname{rk}_{\Gamma_{i}^{\prime}}(p)=\operatorname{rk}_{\Gamma}\left(\alpha_{i}^{-1} p\right)-\operatorname{rk}_{\Gamma_{0}}\left(\alpha_{i}^{-1} p\right)$ for every parabolic fixed point $p$ of $\Gamma_{i}^{\prime}$, since $\alpha_{i}^{-1} p$ is a parabolic fixed point of $\Gamma_{0}$. By the above torsion-free case, for $i=1, \ldots, k$, there exists $c_{i}>0$ such that Card $A_{i}(T) \sim c_{i} T^{\delta}$ as $T \rightarrow+\infty$. The result then follows with $c=\sum_{i=1}^{k} c_{i}$. 
Corollary 18 Let $\Gamma$ be a geometrically finite discrete subgroup of $\mathrm{PSL}_{2}(\mathbb{C})$ with bounded and not totally disconnected limit set in $\mathbb{C}$, which does not contain a quasifuchsian subgroup with index at most 2. Then there exists $c>0$ such that the number of connected components of the domain of discontinuity $\Omega \Gamma$ of $\Gamma$ with diameter at least $1 / T$ is equivalent, as $T \rightarrow+\infty$, to $c T^{\delta}$ where $\delta$ is the Hausdorff dimension of the limit set of $\Gamma$.

When $\infty$ is not the fixed point of an elliptic element of $\Gamma$, we have

$$
c=\frac{2^{\delta}\left\|\sigma_{\mathscr{D}}-\right\|}{\delta\left\|m_{\mathrm{BM}}\right\|} \sum_{\Omega}\left\|\sigma_{\widehat{\Omega}}\right\|
$$

with $\mathscr{D}^{-}=\left(\gamma \mathscr{H}_{\infty}\right)_{\gamma \in \Gamma}$ and $\widehat{\Omega}=(\gamma \mathscr{C} \Omega)_{\gamma \in \Gamma}$, where $\Omega$ ranges over a set of representatives of the $\Gamma$-orbits of the connected components of $\Omega \Gamma$ whose stabiliser has infinite index in $\Gamma$.

Proof. As mentioned after Corollary 17, we have $\delta>1$, hence the assumption of this corollary on the ranks of parabolic groups is satisfied. By Ahlfors's finiteness theorem, the domain of discontinuity $\Omega \Gamma$ of $\Gamma$ (which is a finitely generated Kleinian group) has only finitely many orbits of connected components (see for instance [Kap, Coro. 4.108]). Since $\Gamma$ is geometrically finite, the stabiliser of a component of $\Omega \Gamma$ is again geometrically finite (see for instance [Kap, Coro. 4.112]).

The components of $\Omega \Gamma$ which are stabilised by a finite index subgroup of $\Gamma$ do not contribute to the asymptotics. The assumptions on $\Gamma$ imply that there exists at least one other component of $\Omega \Gamma$. Otherwise indeed, the stabiliser of every component $\Omega$ of $\Omega \Gamma$ has finite index in $\Gamma$, and in particular $\partial \Omega=\Lambda \Gamma$. Up to taking a finite index subgroup, we may assume that $\Gamma$ is a function group (that is, leaves invariant a component of $\Omega \Gamma$ ). By [MaT, Theo. 4.36], $\Gamma$ is a Klein combination of $B$-groups (groups preserving a simply connected component of their domain of discontinuity) and elementary groups. Since $\Lambda \Gamma$ is not totally disconnected and since $\partial \Omega=\Lambda \Gamma$ for all components $\Omega$ of $\Omega \Gamma$, this implies that $\Gamma$ is a $B$-group. By the structure theorem of geometrically finite $B$-groups (see $\mathrm{Abi}$, Theo. 8]), this implies that $\Gamma$ is quasifuchsian, a contradiction. The stabilisers of these other components of $\Omega \Gamma$ have infinite index in $\Gamma$. Hence the result follows from Corollary 17, by a finite summation. 
For example, Corollary 18 gives the asymptotic number of the components of the domain of discontinuity with diameter less than $\frac{1}{T}$ as $T \rightarrow \infty$ of the crossed Fuchsian group generated by two Fuchsian groups (see Chapter VIII $\S \mathrm{E} .8$ of [Mas]) as in the figure below, produced using McMullen's program lim. See for example Maskit's combination theorem in op. cit. for a proof that crossed Fuchsian groups are geometrically finite.

We now consider $\mathbb{H}_{\mathbb{C}}^{n}$, leaving to the reader the extension to the other rank one symmetric spaces. We denote by $\left(w^{\prime}, w\right) \mapsto w^{\prime} \cdot w=\sum_{i=1}^{n-1} w_{i}^{\prime} \overline{w_{i}}$ the usual Hermitian product on $\mathbb{C}^{n-1}$, and $|w|=\sqrt{w \cdot w}$. Let

$$
\mathbb{H}_{\mathbb{C}}^{n}=\left\{\left(w_{0}, w\right) \in \mathbb{C} \times \mathbb{C}^{n-1}: 2 \operatorname{Re} w_{0}-|w|^{2}>0\right\},
$$

endowed with the Riemannian metric (normalised as in the beginning of Section 5)

$$
d s^{2}=\frac{1}{\left(2 \operatorname{Re} w_{0}-|w|^{2}\right)^{2}}\left(\left(d w_{0}-d w \cdot w\right)\left(\left(\overline{d w_{0}}-w \cdot d w\right)+\left(2 \operatorname{Re} w_{0}-|w|^{2}\right) d w \cdot d w\right),\right.
$$

be the Siegel domain model of the complex hyperbolic $n$-space (see [Gol, Sect. 4.1]). Let

$$
\mathscr{H}_{\infty}=\left\{\left(w_{0}, w\right) \in \mathbb{C} \times \mathbb{C}^{n-1}: 2 \operatorname{Re} w_{0}-|w|^{2} \geq 2\right\},
$$

which is a horoball centred at $\infty$. The manifold

$$
\operatorname{Heis}_{2 n-1}=\partial_{\infty} \mathbb{H}_{\mathbb{C}}^{n}-\{\infty\}=\left\{\left(w_{0}, w\right) \in \mathbb{C} \times \mathbb{C}^{n-1}: 2 \operatorname{Re} w_{0}-|w|^{2}=0\right\}
$$

is a Lie group (isomorphic to the $(2 n-1)$-dimensional Heisenberg group) for the law

$$
\left(w_{0}, w\right) \cdot\left(w_{0}^{\prime}, w^{\prime}\right)=\left(w_{0}+w_{0}^{\prime}+w \cdot w^{\prime}, w+w^{\prime}\right) .
$$

The Cygan distance $d_{\text {Cyg }}$ (see [Gol, p. 160]) and the modified Cygan distance $d_{\text {Cyg }}^{\prime}$ (introduced in [PP1, Lem. 6.1]) are the unique left-invariant distances on Heis $2 n-1$ with

$$
d_{\mathrm{Cyg}}\left(\left(w_{0}, w\right),(0,0)\right)=\sqrt{2\left|w_{0}\right|}, \quad d_{\mathrm{Cyg}}^{\prime}\left(\left(w_{0}, w\right),(0,0)\right)=\sqrt{2\left|w_{0}\right|+|w|^{2}} .
$$

Let $d_{\text {Cyg }}^{\prime \prime}=\frac{d_{\mathrm{Cyg}}^{2}}{d_{\text {Cyg }}^{\prime}}$, which, since $d_{\text {Cyg }} \leq d_{\text {Cyg }}^{\prime} \leq \sqrt{2} d_{\text {Cyg }}$, is almost a distance on Heis $2 n-1$. For every nonempty subset $A$ of $\operatorname{Heis}_{2 n-1}$, we denote the "diameter" of $A$ for $d_{\text {Cyg }}^{\prime \prime}$ by

$$
\operatorname{diam}_{d_{\text {Cyg }}^{\prime \prime}}(A)=\max _{x, y \in A: x \neq y} d_{\text {Cyg }}^{\prime \prime}(x, y) .
$$

Corollary 19 Let $\Gamma$ be a discrete nonelementary group of isometries of the Siegel domain model of $\mathbb{H}_{\mathbb{C}}^{n}$, with finite Bowen-Margulis measure $m_{\mathrm{BM}}$. Assume that $\Lambda \Gamma$ is bounded in Heis $_{2 n-1}$, and that $\infty$ is not the fixed point of an elliptic element of $\Gamma$. Let $\mathscr{D}^{-}$be the $\Gamma$-equivariant family $\left(\gamma \mathscr{H}_{\infty}\right)_{\gamma \in \Gamma \text {. Let }}\left(F_{i}\right)_{i \in I}$ be a $\Gamma$-equivariant family of nonempty closed subsets in $\Lambda \Gamma$, whose family $\mathscr{D}^{+}=\left(\mathscr{C} F_{i}\right)_{i \in I}$ of convex hulls in $\mathbb{H}_{\mathbb{C}}^{n}$ is locally finite, with finite nonzero skinning measure. Then, as $T \rightarrow+\infty$,

$$
\operatorname{Card}\left\{i \in I / \sim: \operatorname{diam}_{d_{\text {Cyg }}^{\prime \prime}}\left(F_{i}\right) \geq 1 / T\right\} \sim \frac{\left\|\sigma_{\mathscr{D}}-\right\|\left\|\sigma_{\mathscr{D}+}\right\|}{\delta\left\|m_{\mathrm{BM}}\right\|}(2 T)^{\delta} .
$$

The proof of this corollary is similar to the one of Corollary 16 (1), and has a similar corollary as Corollary 17 (replacing the rank of a parabolic fixed point by twice the critical exponent of its stabiliser), since

- the (signed) length in $\mathbb{H}_{\mathbb{C}}^{n}$ of the common perpendicular from $\mathscr{H}_{\infty}$ to a geodesic in $\mathbb{H}_{\mathbb{C}}^{n}$ with endpoints $x, y \in \operatorname{Heis}_{2 n-1}$ is $\log \frac{2}{d_{\text {Cyg }}^{\prime \prime}(x, y)}$ by [PP2, Lem. 3.4];

- the critical exponent of a geometrically finite group $\Gamma$ of isometries of $\mathbb{H}_{\mathbb{C}}^{n}$ is the Hausdorff dimension of $\Lambda \Gamma$ for any (almost) distance $d_{\mathrm{Cyg}}, d_{\mathrm{Cyg}}^{\prime}$ or $d_{\mathrm{Cyg}}^{\prime \prime}$. 


\section{Counting arcs in finite volume hyperbolic manifolds}

In this subsection, we consider the special case when $M$ is a finite volume complete connected hyperbolic good orbifold. Taking $\widetilde{M}=\mathbb{H}_{\mathbb{R}}^{n}$ to be the ball model of the real hyperbolic space of dimension $n$ and $\Gamma$ to be a discrete group of isometries of $\mathbb{H}_{\mathbb{R}}^{n}$ such that $M$ is isometric to $\Gamma \backslash \mathbb{H}_{\mathbb{R}}^{n}$, the limit set of the group $\Gamma$ is $\mathbb{S}^{n-1}$ and the Patterson-Sullivan density $\left(\mu_{x}\right)_{x \in \mathbb{H}_{\mathbb{R}}^{n}}$ of $\Gamma$ can be normalised such that $\left\|\mu_{x}\right\|=\operatorname{Vol}\left(\mathbb{S}^{n-1}\right)$ for all $x \in \mathbb{H}_{\mathbb{R}}^{n}$.

The Bowen-Margulis measure $m_{\mathrm{BM}}$ is, by homogeneity in this special case, a constant multiple of the Liouville measure $\operatorname{Vol}_{T^{1} M}$ of $T^{1} M$. This measure disintegrates as

$$
d \mathrm{Vol}_{T^{1} M}=\int_{x \in M} d \mathrm{Vol}_{T_{x}^{1} M} d \operatorname{Vol}_{M}(x) .
$$

Note that $\operatorname{Vol}\left(T_{x}^{1} M\right)=\frac{\operatorname{Vol}\left(\mathbb{S}^{n-1}\right)}{\operatorname{Card}\left(\Gamma_{\widetilde{x}}\right)}$ where $\Gamma_{\widetilde{x}}$ is the stabiliser in $\Gamma$ of any lift $\widetilde{x}$ of $x$ in $\mathbb{H}_{\mathbb{R}}^{n}$, and that $\Gamma_{\widetilde{x}}=\{e\}$ for $\operatorname{Vol}_{M}$-almost every $x \in M$. Furthermore, if $D$ is a totally geodesic subspace or a horoball in $\mathbb{H}_{\mathbb{R}}^{n}$, then the skinning measures $\sigma_{D}^{ \pm}$are, again by homogeneity, constant multiples of the induced Riemannian measures $\operatorname{Vol}_{\partial_{ \pm}^{1}} D$. These measures disintegrate with respect to the basepoint fibration $\partial_{ \pm}^{1} D \rightarrow \partial D$ over the Riemannian measure of the boundary $\partial D$ of $D$ in $\mathbb{H}_{\mathbb{R}}^{n}$ with measure on the fiber of $x \in \partial D$ the spherical measure on the outer/inner unit normal vectors to $D$ at $x$ :

$$
d \operatorname{Vol}_{\partial_{ \pm}^{1} D}=\int_{x \in \partial D} d \operatorname{Vol}_{\partial_{+}^{1} D \cap T_{x}^{1} M} d \operatorname{Vol}_{\partial D}(x)
$$

The following result gives the proportionality constants of the various measures explicitly.

Proposition 20 Let $M=\Gamma \backslash \mathbb{H}_{\mathbb{R}}^{n}$ be a finite volume orbifold of dimension $n \geq 2$. Normalise the Patterson-Sullivan density $\left(\mu_{x}\right)_{x \in \mathbb{H}_{\mathbb{R}}^{n}}$ such that $\left\|\mu_{x}\right\|=\operatorname{Vol}\left(\mathbb{S}^{n-1}\right)$ for all $x \in \mathbb{H}_{\mathbb{R}}^{n}$.

(1) We have $m_{\mathrm{BM}}=2^{n-1} \mathrm{Vol}_{T^{1} M}$. In particular,

$$
\left\|m_{\mathrm{BM}}\right\|=2^{n-1} \operatorname{Vol}\left(\mathbb{S}^{n-1}\right) \operatorname{Vol}(M) .
$$

(2) If $D$ is a horoball in $\mathbb{H}_{\mathbb{R}}^{n}$, then $\widetilde{\sigma}_{D}^{ \pm}=2^{n-1} \operatorname{Vol}_{\partial_{ \pm}^{1} D}$. In particular, if $D$ is centered at a parabolic fixed point of $\Gamma$ with stabiliser $\Gamma_{D}$ and if $\mathscr{D}=(\gamma D)_{\gamma \in \Gamma}$, then

$$
\left\|\sigma_{\mathscr{D}}^{ \pm}\right\|=2^{n-1} \operatorname{Vol}\left(\Gamma_{D} \backslash \partial_{ \pm}^{1} D\right)=2^{n-1}(n-1) \operatorname{Vol}\left(\Gamma_{D} \backslash D\right) .
$$

(3) If $D$ is a totally geodesic submanifold of $\mathbb{H}_{\mathbb{R}}^{n}$ with dimension $k \in\{1, \ldots, n-1\}$, then $\widetilde{\sigma}_{D}^{+}=\widetilde{\sigma}_{D}^{-}=\operatorname{Vol}_{\partial_{ \pm}^{1} D}$. In particular, with $\Gamma_{D}$ the stabiliser in $\Gamma$ of $D$, if $\Gamma_{D} \backslash D$ is a properly immersed finite volume suborbifold of $M$ and if $\mathscr{D}=(\gamma D)_{\gamma \in \Gamma}$, then

$$
\left\|\sigma_{\mathscr{D}}^{ \pm}\right\|=\operatorname{Vol}\left(\Gamma_{D} \backslash \partial_{ \pm}^{1} D\right)
$$

If $m$ is the number of elements of $\Gamma$ that pointwise fix $D$, then

$$
\left\|\sigma_{\mathscr{D}}^{ \pm}\right\|=\frac{1}{m} \operatorname{Vol}\left(\mathbb{S}^{n-k-1}\right) \operatorname{Vol}\left(\Gamma_{D} \backslash D\right) .
$$


Proof. Claims (1) and (3) are proven assuming that $\Gamma$ has no torsion in Proposition 10 and Claim (1) of Proposition 11 in [PP8, respectively. If $\Gamma$ has torsion, Claim (1) follows by restricting to the complement of the points in $\widetilde{M}$ with nontrivial stabiliser, this set has zero Riemannian measure in $\widetilde{M}$, and Claim (3) follows from the fact that the fixed point set on $D$ of an isometry which preserves $D$, but does not pointwise fix $D$, has measure 0 for the Riemannian measure of $D$.

The first part of Claim (2) is proved in Claim (1) of [PP8, Prop. 10] for the outer skinning measure. For the second part, note that if the horoball $D$ is precisely invariant (that is, the interiors of $D$ and $\gamma D$ intersect for $\gamma \in \Gamma$ only if $\gamma \in \Gamma_{D}$ ), then $\Gamma_{D} \backslash D$ embeds in $M$ and the image is, by definition, a Margulis cusp neighbourhood. In the general case, there is a precisely embedded horoball $D^{\prime}$ contained in $D$ such that $D=\mathscr{N}_{t} D^{\prime}$ for some $t \geq 0$. Let $\mathscr{D}^{\prime}=\left(\gamma D^{\prime}\right)_{\gamma \in \Gamma}$. As $\Gamma_{D^{\prime}}=\Gamma_{D}$, we have

$$
\left\|\sigma_{\mathscr{D}}^{ \pm}\right\|=e^{(n-1) t}\left\|\sigma_{\mathscr{D}^{\prime}}^{ \pm}\right\|=e^{(n-1) t} 2^{n-1}(n-1) \operatorname{Vol}\left(\Gamma_{D^{\prime}} \backslash D^{\prime}\right)=2^{n-1}(n-1) \operatorname{Vol}\left(\Gamma_{D} \backslash D\right),
$$

by [PP5, Prop. 4 (iii)], by [PP8, Prop. 10] and by the scaling of hyperbolic volume. The case with torsion follows as in Claims (1) and (3).

Proposition 20 allows us to obtain very explicit versions of Theorems 1 and 3 in the case when $M$ is a finite volume hyperbolic manifold (or good orbifold) and the properly immersed closed locally convex subsets are any combination of points, totally geodesic orbifolds or Margulis neighbourhoods of cusps. The following result gives these explicit asymptotics of the counting function in the cases that we have not found in the literature. We refer to the Introduction as well as to our survey [PP8] for more details and references.

Corollary 21 Let $\Gamma$ be a discrete group of isometries of $\mathbb{H}_{\mathbb{R}}^{n}$ such that $M=\Gamma \backslash \mathbb{H}_{\mathbb{R}}^{n}$ has finite volume. If $A^{-}$and $A^{+}$are properly immersed finite volume totally geodesic suborbifolds in $M$ of dimensions $k^{-}$and $k^{+}$in $\{1, \ldots, n-1\}$, respectively, let

$$
c\left(A^{-}, A^{+}\right)=\frac{\operatorname{Vol}\left(\mathbb{S}^{n-k^{-}-1}\right) \operatorname{Vol}\left(\mathbb{S}^{n-k^{+}-1}\right)}{2^{n-1}(n-1) \operatorname{Vol}\left(\mathbb{S}^{n-1}\right)} \frac{\operatorname{Vol}(M)}{\operatorname{Vol}\left(A^{+}\right)} .
$$

If $A^{-}$and $A^{+}$are Margulis cusp neighbourhoods in $M$, let

$$
c\left(A^{-}, A^{+}\right)=\frac{2^{n-1}(n-1) \operatorname{Vol}\left(A^{-}\right) \operatorname{Vol}\left(A^{+}\right)}{\operatorname{Vol}\left(\mathbb{S}^{n-1}\right) \operatorname{Vol}(M)} .
$$

If $A^{-}$is a point and $A^{+}$is a Margulis cusp neighbourhood, let

$$
c\left(A^{-}, A^{+}\right)=\frac{\operatorname{Vol}\left(A^{+}\right)}{\operatorname{Vol}(M)} .
$$

If $A^{-}$is a Margulis cusp neighbourhood and $A^{+}$is a properly immersed finite volume totally geodesic suborbifold in $M$ of dimension $k$ in $\{1, \ldots, n-1\}$, let

$$
c\left(A^{-}, A^{+}\right)=\frac{\operatorname{Vol}\left(\mathbb{S}^{n-1-k}\right) \operatorname{Vol}\left(A^{-}\right) \operatorname{Vol}\left(A^{+}\right)}{\operatorname{Vol}\left(\mathbb{S}^{n-1}\right) \operatorname{Vol}(M)} .
$$

In each of these cases, if $m^{ \pm}$is the cardinality of the intersection of the isotropy groups in the orbifold $M$ of the points of $A^{ \pm}$, then

$$
\mathscr{N}_{A^{-}, A^{+}}(t)=\mathscr{N}_{A^{-}, A^{+}, 0}(t) \sim \frac{c\left(A^{-}, A^{+}\right)}{m^{-} m^{+}} e^{(n-1) t} .
$$


Furthermore, if $\Gamma$ is arithmetic or if $M$ is compact, then there is some $\kappa^{\prime \prime}>0$ such that, as $t \rightarrow+\infty$,

$$
\mathscr{N}_{A^{-}, A^{+}}(t)=\frac{c\left(A^{-}, A^{+}\right)}{m^{-} m^{+}} e^{(n-1) t}\left(1+\mathrm{O}\left(e^{-\kappa^{\prime \prime} t}\right)\right)
$$

We refer to [PP6] for several new arithmetic applications of these results.

\section{References}

[Abi] W. Abikoff. On boundaries of Teichmüller spaces and on Kleinian groups III. Acta Math. 134 (1975) 211-237.

[Bab] M. Babillot. On the mixing property for hyperbolic systems. Israel J. Math. 129 (2002) $61-76$.

[Bal] W. Ballmann. Lectures on spaces of nonpositive curvature. DMW Seminar 25, Birkhäuser, 1995.

[Bea] A. F. Beardon. The geometry of discrete groups. Grad. Texts Math. 91, Springer-Verlag, 1983.

[Bow] B. Bowditch. Geometrical finiteness with variable negative curvature. Duke Math. J. 77 (1995) 229-274.

[BrH] M. R. Bridson and A. Haefliger. Metric spaces of non-positive curvature. Grund. math. Wiss. 319, Springer Verlag, 1999.

[Bri] M. Brin. Ergodicity of the geodesic flow. Appendix in W. Ballmann, Lectures on spaces of nonpositive curvature, DMV Seminar 25, Birkhäuser, 1995, 81-95.

[BPP] A. Broise-Alamichel and J. Parkkonen and F. Paulin. Equidistribution and counting in negatively curved spaces and quantum graphs. Applications to non-Archimedian Diophantine approximation. Book in preparation.

[CaT] R.D. Canary and E. Taylor. Kleinian groups with small limit sets. Duke Math. J. 73 (1994) 371-381.

[Clo] L. Clozel. Démonstration de la conjecture $\tau$. Invent. Math. 151 (2003) 297-328.

[Cos] S. Cosentino. Equidistribution of parabolic fixed points in the limit set of Kleinian groups. Erg. Theo. Dyn. Syst. 19 (1999) 1437-1484.

[Dal1] F. Dal'Bo. Remarques sur le spectre des longueurs d'une surface et comptage. Bol. Soc. Bras. Math. 30 (1999) 199-221.

[Dal2] F. Dal'Bo. Topologie du feuilletage fortement stable. Ann. Inst. Fourier 50 (2000) 981-993.

[DOP] F. Dal'Bo, J.-P. Otal, and M. Peigné. Séries de Poincaré des groupes géométriquement finis. Israel J. Math. 118 (2000) 109-124.

[Dol] D. Dolgopyat. On decay of correlation in Anosov flows. Ann. of Math. 147 (1998) 357390 .

[EM] A. Eskin and C. McMullen. Mixing, counting, and equidistribution in Lie groups. Duke Math. J. 71 (1993) 181-209.

[GLP] P. Giulietti, C. Liverani, and M. Pollicott. Anosov flows and dynamical zeta functions. Ann. of Math. 178 (2013) 687-773.

[Gol] W. M. Goldman. Complex hyperbolic geometry. Oxford Univ. Press, 1999. 
[Ham] U. Hamenstädt. A new description of the Bowen-Margulis measure. Erg. Theo. Dyn. Sys. 9 (1989) 455-464.

[Her] O. Herrmann. Über die Verteilung der Längen geodätischer Lote in hyperbolischen Raumformen. Math. Z. 79 (1962) 323-343.

[HP1] S. Hersonsky and F. Paulin. On the rigidity of discrete isometry groups of negatively curved spaces. Comm. Math. Helv. 72 (1997) 349-388.

[HP2] S. Hersonsky and F. Paulin. Counting orbit points in coverings of negatively curved manifolds and Hausdorff dimension of cusp excursions. Erg. Theo. Dyn. Sys. 24 (2004) 803-824.

[Hub1] H. Huber. Über eine neue Klasse automorpher Funktionen und ein Gitterpunktproblem in der hyperbolischen Ebene. I. Comment. Math. Helv. 30 (1956) 20-62.

[Hub2] H. Huber. Zur analytischen Theorie hyperbolischen Raumformen und Bewegungsgruppen. Math. Ann. 138 (1959) 1-26.

[Kap] M. Kapovich. Hyperbolic manifolds and discrete groups. Prog. Math. 183, Birkhäuser, 2001.

[Kim] I. Kim. Counting, mixing and equidistribution of horospheres in geometrically finite rank one locally symmetric manifolds. Preprint [arXiv:1103.5003], to appear in J. reine angew. Mathematik.

[KM1] D. Kleinbock and G. Margulis. Bounded orbits of nonquasiunipotent flows on homogeneous spaces. Sinai's Moscow Seminar on Dynamical Systems, 141-172, Amer. Math. Soc. Transl. Ser. 171, Amer. Math. Soc. 1996.

[KM2] D. Kleinbock and G. Margulis. Logarithm laws for flows on homogeneous spaces. Invent. Math. 138 (1999) 451-494.

[Kon] A. Kontorovich. The hyperbolic lattice point count in infinite volume with applications to sieves. Duke Math. J. 149 (2009) 1-36.

[KO] A. Kontorovich and H. Oh. Apollonian circle packings and closed horospheres on hyperbolic 3-manifolds. J. Amer. Math. Soc. 24 (2011) 603-648.

[LaP] P. D. Lax and R. S. Phillips. The asymptotic distribution of lattice points in Euclidean and non-Euclidean spaces. J. Funct. Anal. 46 (1982) 280-350.

[LO] M. Lee and H. Oh. Effective circle count for Apollonian packings and closed horospheres. Geom. Funct. Anal. 23 (2013) 580-621.

[Mar] G. Margulis. Applications of ergodic theory for the investigation of manifolds of negative curvature. Funct. Anal. Applic. 3 (1969) 335-336.

[MaMW] K. Martin, M. McKee, and E. Wambach. A relative trace formula for a compact Riemann surface. Int. J. Number Theory 7 (2011) 389-429; see webpage of first author for an erratum.

[Mas] B. Maskit. Kleinian groups. Grund. math. Wiss. 287, Springer-Verlag, 1988.

[MaT] K. Matsuzaki and M. Taniguchi. Hyperbolic manifolds and Kleinian groups. Oxford Univ. Press, 1998.

[MO] A. Mohammadi and H. Oh. Matrix coefficients, counting and primes for orbits of geometrically finite groups. J. Euro. Math. Soc. 17 (2015) 837-897.

[Moo] C. Moore. Exponential decay of correlation coefficients for geodesic flows. In "Group representations, ergodic theory, operator algebras, and mathematical physics" (Berkeley, 1984), 163-181, Math. Sci. Res. Inst. Publ. 6, Springer, 1987. 
[OS1] H. Oh and N. Shah. The asymptotic distribution of circles in the orbits of Kleinian groups. Invent. Math. 187 (2012) 1-35.

[OS2] H. Oh and N. Shah. Equidistribution and counting for orbits of geometrically finite hyperbolic groups. J. Amer. Math. Soc. 26 (2013) 511-562.

[OS3] H. Oh and N. Shah. Counting visible circles on the sphere and Kleinian groups. Preprint [arXiv: 1004.2129], to appear in "Geometry, Topology and Dynamics in Negative Curvature" (ICM 2010 satellite conference, Bangalore), C. S. Aravinda, T. Farrell, J.-F. Lafont eds, London Math. Soc. Lect. Notes.

[PP1] J. Parkkonen and F. Paulin. Prescribing the behaviour of geodesics in negative curvature. Geom. \& Topo. 14 (2010) 277-392.

[PP2] J. Parkkonen and F. Paulin. Spiraling spectra of geodesic lines in negatively curved manifolds. Math. Z. 268 (2011) 101-142, Erratum: Math. Z. 276 (2014) 1215-1216.

[PP3] J. Parkkonen and F. Paulin. Équidistribution, comptage et approximation par irrationnels quadratiques. J. Mod. Dyn. 6 (2012) 1-40.

[PP4] J. Parkkonen and F. Paulin. On strictly convex subsets in negatively curved manifolds. J. Geom. Analysis 22 (2012) 621-632.

[PP5] J. Parkkonen and F. Paulin. Skinning measure in negative curvature and equidistribution of equidistant submanifolds. Erg. Theo. Dyn. Sys. 34 (2014) 1310-1342.

[PP6] J. Parkkonen and F. Paulin. On the arithmetic of crossratios and generalised Mertens' formulas. Numéro Spécial "Aux croisements de la géométrie hyperbolique et de l'arithmétique", F. Dal'Bo, C. Lecuire eds, Ann. Fac. Scien. Toulouse 23 (2014) 9671022.

[PP7] J. Parkkonen and F. Paulin. On the hyperbolic orbital counting problem in conjugacy classes. Math. Z. 279 (2015) 1175-1196.

[PP8] J. Parkkonen and F. Paulin. Counting arcs in negative curvature. Preprint [arXiv:1203. 0175], to appear in "Geometry, Topology and Dynamics in Negative Curvature" (ICM 2010 satellite conference, Bangalore), C. S. Aravinda, T. Farrell, J.-F. Lafont eds, London Math. Soc. Lect. Notes.

[PP9] J. Parkkonen and F. Paulin. Counting and equidistribution in Heisenberg groups. Preprint [hal-00955576], [arXiv: 1402.7225].

[PPS] F. Paulin, M. Pollicott, and B. Schapira. Equilibrium states in negative curvature. Book preprint (281 pages) [arXiv: 1211.6242], to appear in Astérisque 373, Soc. Math. France.

[Pol] M. Pollicott. The Schottky-Klein prime function and counting functions for Fenchel double crosses. Preprint 2011.

[Rob] T. Roblin. Ergodicité et équidistribution en courbure négative. Mémoire Soc. Math. France, 95 (2003).

[Wal] R. Walter. Some analytical properties of geodesically convex sets. Abh. Math. Sem. Univ. Hamburg 45 (1976) 263-282.

Department of Mathematics and Statistics, P.O. Box 35

40014 University of Jyväskylä, FINLAND.

e-mail: jouni.t.parkkonen@jyu.fi

Département de mathématique, UMR 8628 CNRS, Bât. 425

Université Paris-Sud, 91405 ORSAY Cedex, FRANCE

e-mail: frederic.paulin@math.u-psud.fr 